\title{
Cloud Implementation of Design Software for Strengthening of Reinforced Concrete Member
}

\author{
Sucharitha Koleti
}

Follow this and additional works at: https://researchrepository.wvu.edu/etd

\section{Recommended Citation}

Koleti, Sucharitha, "Cloud Implementation of Design Software for Strengthening of Reinforced Concrete Member" (2015). Graduate Theses, Dissertations, and Problem Reports. 6002.

https://researchrepository.wvu.edu/etd/6002

This Thesis is protected by copyright and/or related rights. It has been brought to you by the The Research Repository @ WVU with permission from the rights-holder(s). You are free to use this Thesis in any way that is permitted by the copyright and related rights legislation that applies to your use. For other uses you must obtain permission from the rights-holder(s) directly, unless additional rights are indicated by a Creative Commons license in the record and/ or on the work itself. This Thesis has been accepted for inclusion in WVU Graduate Theses, Dissertations, and Problem Reports collection by an authorized administrator of The Research Repository @ WVU. For more information, please contact researchrepository@mail.wvu.edu. 
Cloud Implementation of Design Software for Strengthening of Reinforced Concrete Member

\author{
Sucharitha Koleti
}

Thesis submitted to Benjamin M. Statler College of Engineering and Mineral Resources at West Virginia University in partial fulfillment of the requirements for the degree of

Master of Science

In

Mechanical Engineering

\author{
Ever J. Barbero, Ph.D., Chair \\ Victor H. Mucino, Ph.D. \\ Eduardo M. Sosa, Ph.D.
}

Department of Mechanical and Aerospace Engineering

\author{
Morgantown, West Virginia
}

April, 2015

Keywords: Fiber Reinforced Composite; Strengthening of RC; CADEC 


\begin{abstract}
Cloud Implementation of Design Software for Strengthening of Reinforced Concrete Member
\end{abstract}

\title{
Sucharitha Koleti
}

The design of Fiber Reinforced Composite (FRP) which has emerged as an effective alternative to strengthen concrete structures, using traditional approaches, involves a set of complex design procedures to strengthen a concrete member. The design procedures are classified as a three step processes to build an intuitive and user friendly web application. The design software incorporated in the aforementioned application solves for strengthening of Reinforced Concrete (RC) Beams and Columns. This application has been developed as an addon to the existing Computer Aided Design Environment to Composites (CADEC). It has been built on the .Net platform in compliance with the CADEC framework.

The application facilitates the design strengthening of FRP under shear and flexure for $\mathrm{RC}$ beam members and axial stresses for column members respectively. In the three step design process, the first two steps involve inputting parameters, while the final step involves the discussion of checks and results concerning the respective member. The validity of the application has also been tested using illustrated examples.

Cloud implementation increases usability by making the application machine independent, while letting the user to create and save FRP designs. User data is managed by the database built using SQL Server. The application thus allows the design strengthening of FRP for basic members of a structure with ease while allowing the user to manage designs with in the confines of the application. 


\section{Table of Contents}

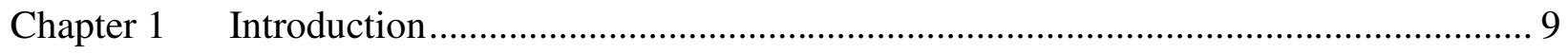

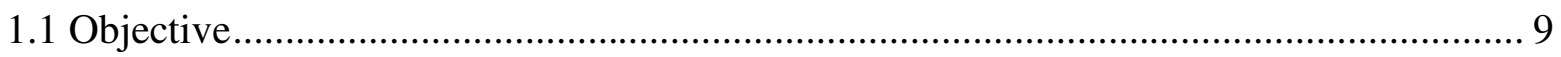

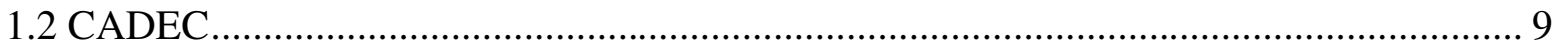

Chapter 2 Strengthening of Reinforced Concrete Members ………………………................ 11

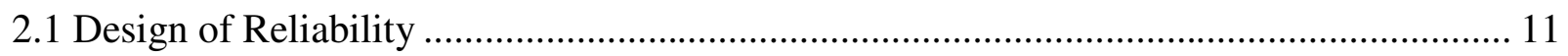

2.2 FRP Load and Strength Design ................................................................................... 11

2.3 Environmental Reduction Factor ………………….................................................. 12

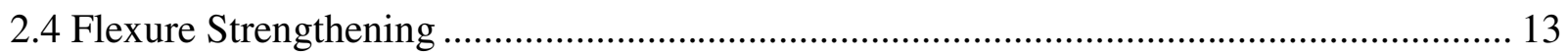

2.5 Shear Strengthening ……………………………................................................... 16

Chapter $3 \quad$ The Microsoft .NET Framework …………............................................................ 19

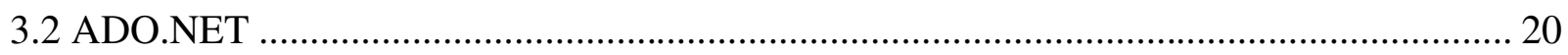

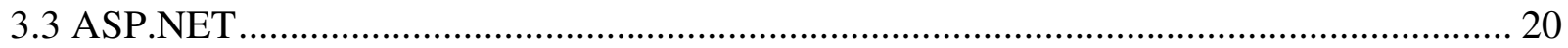

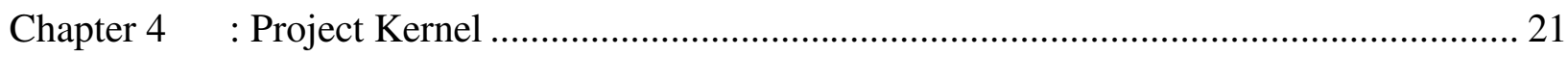

4.1 Design Software Methods ........................................................................................... 21

4.1.1 Class: ClsLoading ................................................................................................... 22

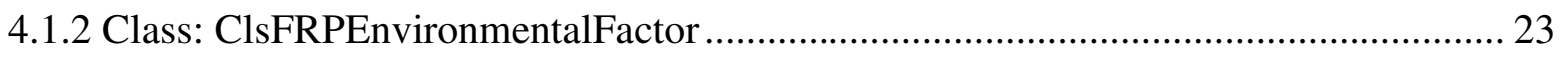

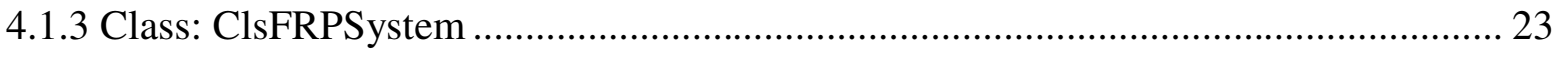

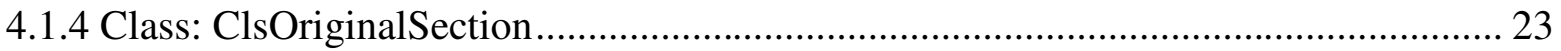

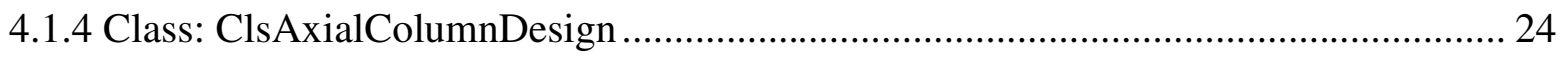

4.1.5 Class: ClsAxialColumnACIRequirements ................................................................. 25

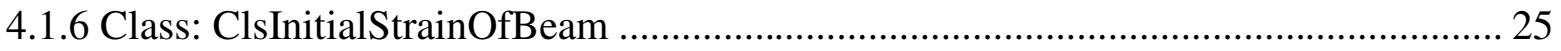

4.1.7 Class: Class- ClsFlexureFRPDesign ..................................................................... 26

4.1.8 Class: Class- ClsFlexureACIRequirements .......................................................... 26

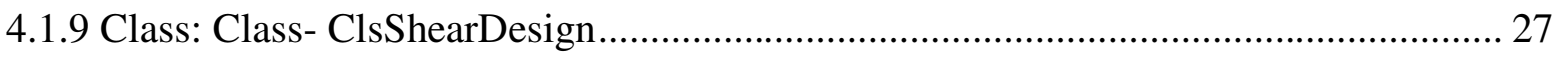

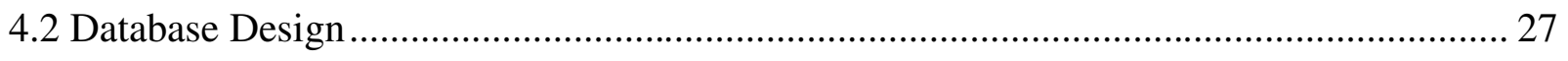

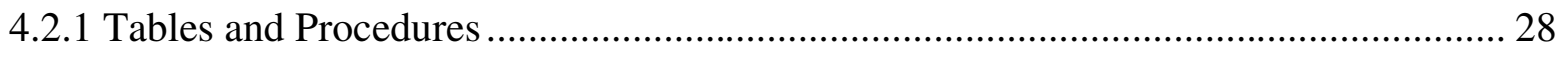

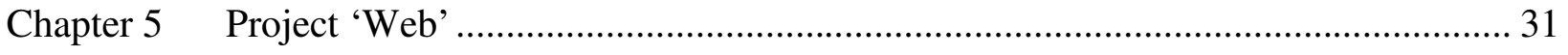

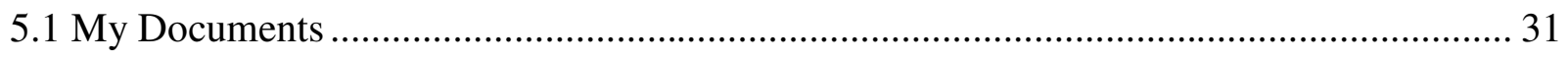

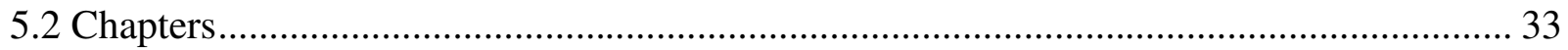

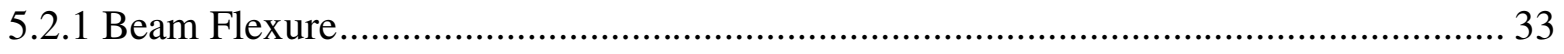




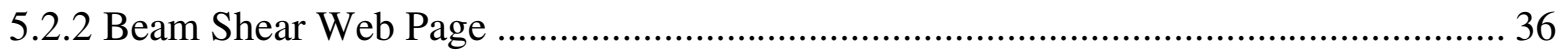

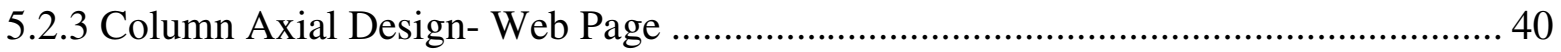

5.2.3 Beam-Column Axial Design- Web Page ..................................................................... 43

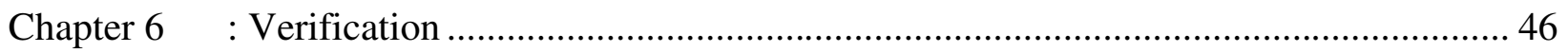

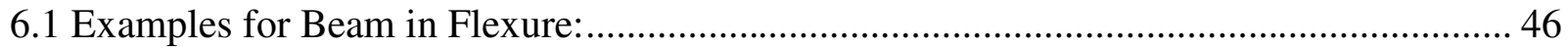

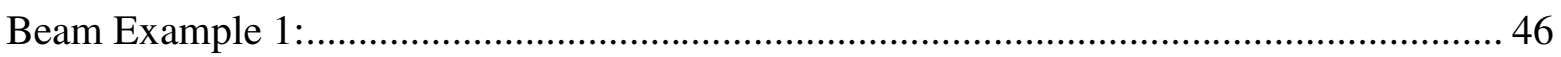

Chapter $7 \quad$ : Conclusions and Future Work …………........................................................ 57

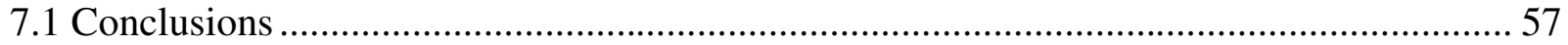

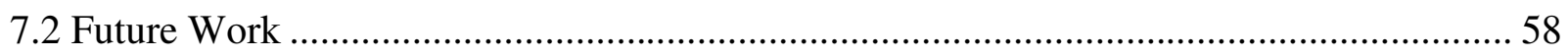

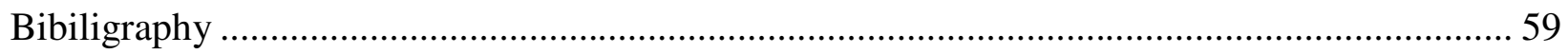

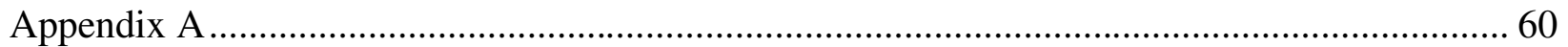

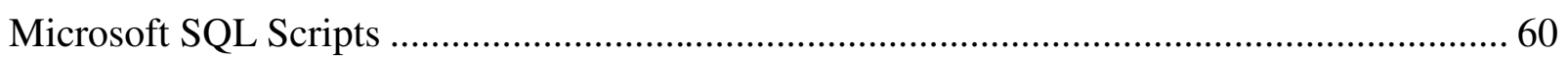

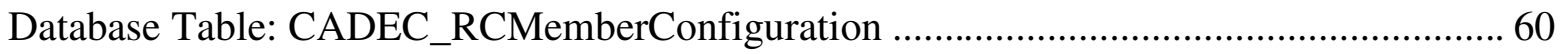

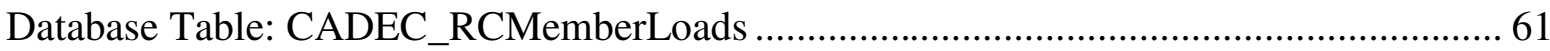

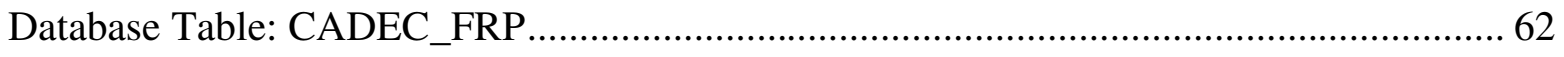

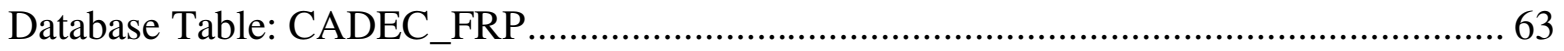

Stored Procedure: CADEC_RCMemberConfiguration_Insert ............................................... 64

Stored Procedure: CADEC_RCMemberConfiguration_Update …………………….............. 65

Stored Procedure: CADEC_RCMemberConfiguration_ Delete ……………………….......... 67

Stored Procedure: CADEC_RCMemberConfiguration_Get_One ......................................... 67

Stored Procedure: CADEC_RCMemberConfiguration_Get_One ......................................... 68

Stored Procedure: CADEC_RCMemberConfiguration_Get_One ......................................... 69

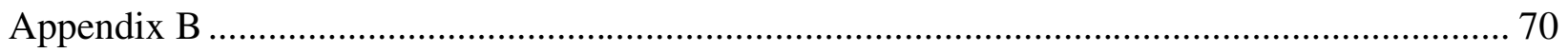

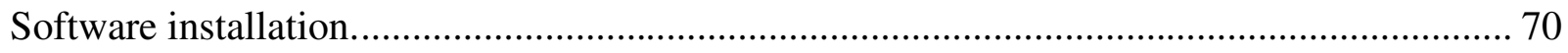

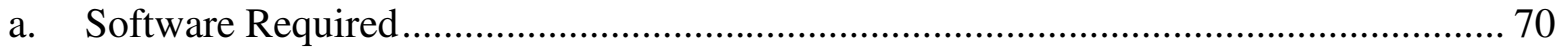

b. Installation Procedure for Microsoft SQL Server 2008 .................................................... 70

c. Installation Procedure for Microsoft SQL Server 2008 SP4 ……………………........ 80

d. Installation Procedure for Microsoft SQL Management Studio 2008 ............................ 84

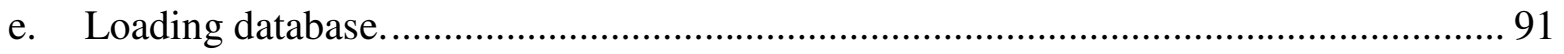

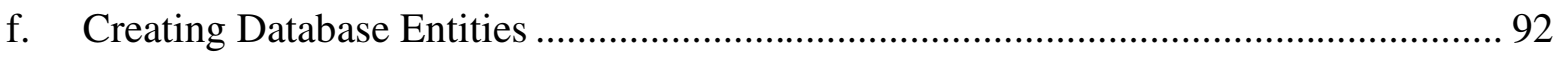

g. Configure Database in Visual Studio and store RC Member Properties. ....................... 93 


\section{Table of Figures}

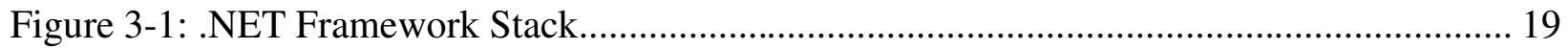

Figure 4-1: Software methods in Kernel namespace ........................................................... 22

Figure 4-2: Class Diagram- ClsLoading ............................................................................ 22

Figure 4-3: Class Diagram- ClsFRPEnvironmentalFactor .................................................. 23

Figure 4-4: Class Diagram- ClsFRPSystem ................................................................ 23

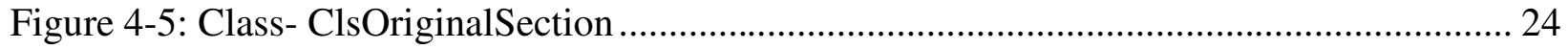

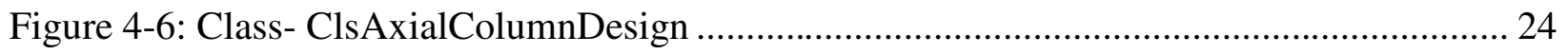

Figure 4-7: Class- ClsAxialColumnACIRequirements ..................................................... 25

Figure 4-8: Class- ClsInitialStrainOfBeam.................................................................... 25

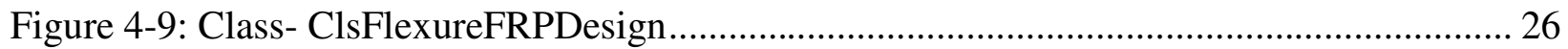

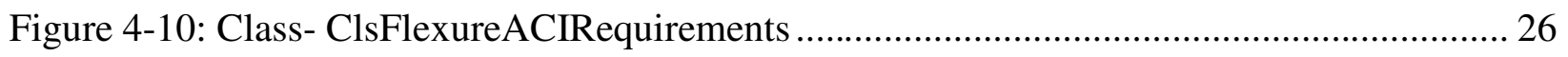

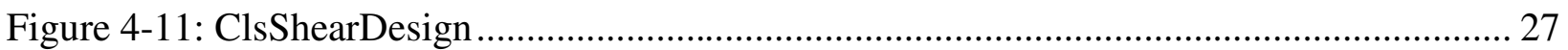

Figure 4-12: Table schema of CADEC_RCMemberProperties,

CADEC_BeamColumnSteelProperties and their foreign key relation .................................... 28

Figure 4-13: Table Schema- CADEC_RCMemberLoads ...................................................... 29

Figure 4-14: Table Schema- CADEC_FRP ......................................................................... 29

Figure 5-1: Page corresponding to table 'CADEC_RCMemberLoads' ..................................... 32

Figure 5-2: View of all the records from 'CADEC_RCMemberLoads' in the web page............. 33

Figure 5-3: Step 1 of Beam Flexure Design .............................................................................. 34

Figure 5-4: $2^{\text {nd }}$ Step of Beam Flexure Strengthening- Defining properties of the member......... 34

Figure 5-5: $3^{\text {rd }}$ Step of Beam Flexure Strengthening-Initial Checks. ....................................... 35

Figure 5-6: $3^{\text {rd }}$ Step of Beam Flexure Strengthening- Serviceability Checks............................ 36

Figure 5-7: $1^{\text {st }}$ step in Beam Shear Strengthening- Defining load configuration ........................ 37

Figure 5-8: $2^{\text {nd }}$ Step in Beam Shear Strengthening - Defining properties of the member ........... 38

Figure 5-9: $3^{\text {rd }}$ step in Beam Shear Strengthening - Initial checks.......................................... 39

Figure 5-10: $3^{\text {rd }}$ Step in Beam Shear Design and Analysis- Serviceability Checks .................... 40

Figure 5-11: $1^{\text {st }}$ step in Column Axial Strengthening- Defining Loading Configuration............ 40

Figure 5-12: $2^{\text {nd }}$ step of Column Axial Load Design - Defining properties of the member. ...... 41

Figure 5-13: $3^{\text {rd }}$ Step in Column Axial Strengthening Design- Initial Checks ........................... 42

Figure 5-14: $3^{\text {rd }}$ step in Column Axial Strengthening- Serviceability checks ............................ 43

Figure 5-15: 1st step in Beam-Column Strengthening- Defining Loading Configuration .......... 43

Figure 5-16: 2nd step in Beam-Column Strengthening- Defining Member Configuration.......... 44

Figure 5-17: 3rd step in Beam-Column Strengthening- Verification ...................................... 45

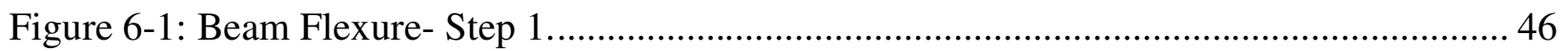

Figure 6-2: Beam Flexure- Step 2 ............................................................................... 47

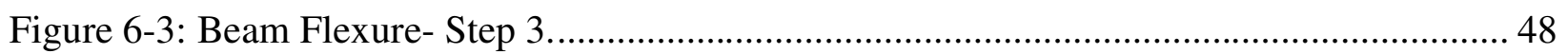

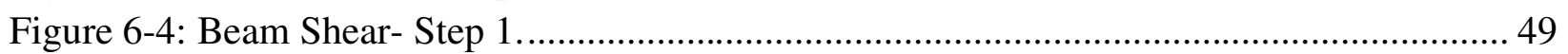

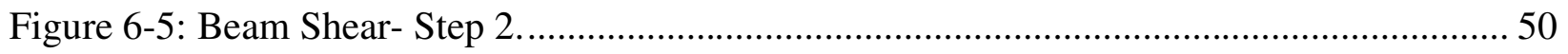

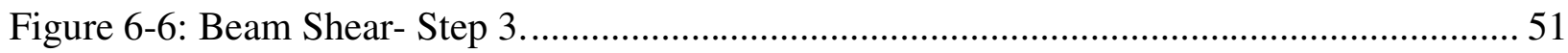




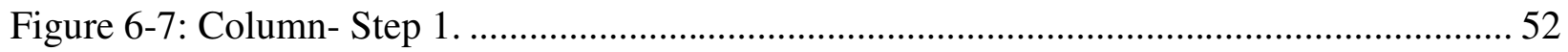

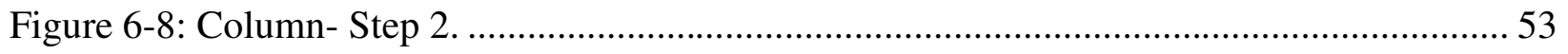

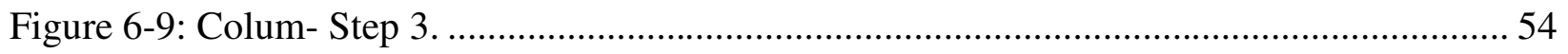

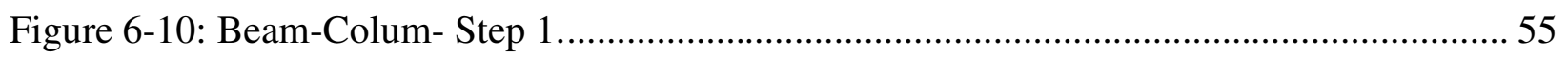

Figure 6-11: Beam-Colum- Step 2 .......................................................................... 55

Figure 6-12: Beam-Colum- Step 3 ............................................................................... 56

Figure 7-1: Picture of www.cadec-online.com home page showing status of Chapter 13-

'Strengthening Reinforced Concrete' ................................................................................ 58 


\section{List of Symbols}

$$
\begin{aligned}
& \alpha \quad=\text { angle of shear steel reinforcement } \\
& \alpha_{\mathrm{f}} \quad=\text { angle of shear FRP } \\
& \beta_{1} \quad=\text { stress block parameter for unconfined section } \\
& \varepsilon_{f u}^{*} \quad=\text { FRP ultimate strain } \\
& \varepsilon_{c}^{\prime} \quad=\text { maximum strain of unconfined concrete }=0.002 \\
& \varepsilon_{b i} \quad=\text { Initial strain at the soffit } \\
& \varepsilon_{c c u}=\text { ultimate axial compressive strain of confined concrete } \\
& \varepsilon_{c u}=\text { ultimate axial strain of confined concrete } \\
& \varepsilon_{f} \quad=\text { strain in FRP } \\
& \varepsilon_{f d} \quad=\text { FRP debonding strain } \\
& \varepsilon_{f e} \quad=\text { effective FRP strain } \\
& \varepsilon_{f u} \quad=\text { FRP allowable tensile strain } \\
& \varepsilon_{s y}=\text { strain corresponding to yield strength of non pre stressed reinforcement } \\
& \varepsilon_{t}, \varepsilon_{s}=\text { net tensile strain in extreme tension steel at nominal strength } \\
& K_{a} \quad=\text { FRP efficiency factor in determination of } f_{c c}^{\prime} \\
& K_{b} \quad=\text { FRP efficiency factor in determination of } \varepsilon_{c c u} \\
& K_{e} \quad=\text { FRP efficiency factor }=0.55 \\
& K_{v} \quad=\text { Bond reduction factor } \\
& \lambda=\text { modification factor } \\
& \rho_{g} \quad=\text { Longitudinal steel reinforcement ratio } \\
& \varphi \quad=\text { strength reduction factor } \\
& \varphi_{e c c}=\text { eccentricity factor } \\
& \psi_{f} \quad=\text { FRP strength reduction factor }
\end{aligned}
$$




$$
\begin{aligned}
& C \quad=\text { position of the neutral axis } \\
& c_{b} \quad=\text { position of the neutral axis for BSC } \\
& d \quad=\text { depth of tensile steel } \\
& d^{\prime} \quad=\text { depth of compressive steel } \\
& d_{f}, d_{f v}=\text { depth of FRP shear strengthening } \\
& e \quad=\text { Eccentricity } \\
& f_{c}^{\prime}=\text { concrete compressive strength of unconfined section } \\
& f_{c c}^{\prime}=\text { concrete compressive strength of confined section } \\
& f_{c, S} \quad=\text { concrete stress } \\
& f_{f, s} \quad=\text { FRP stress } \\
& f_{f e} \quad=\text { FRP effective tensile strength } \\
& f_{f u} \quad=\text { ultimate tensile strength of FRP } \\
& f_{f u}^{*} \quad=\text { FRP tensile strength } \\
& f_{l} \quad=\text { confining pressure } \\
& f_{S, S} \quad=\text { steel stress } \\
& f_{y} \quad=\text { yield strength of steel reinforcement } \\
& h \quad=\text { height of the member } \\
& n \quad=\text { number of layers } \\
& r_{c} \quad=\text { radius of edges of a prismatic cross-section confined with FRP } \\
& S_{S} \quad=\text { steel spacing in shear } \\
& t_{f} \quad=\text { ply thickness } \\
& A_{c} \quad=\text { area of concrete in compression } \\
& A_{e} \quad=\text { area of effectively confined concrete } \\
& A_{f} \quad=\text { area of FRP reinforcement }
\end{aligned}
$$




$$
\begin{aligned}
& A_{f b} \quad=\text { area of FRP for BSC } \\
& A_{g} \quad=\text { gross cross sectional area } \\
& A_{S} \quad=\text { area of tensile steel reinforcement } \\
& A_{s v} \quad=\text { area of shear steel reinforcement } \\
& A_{S}^{\prime} \quad=\text { compressive steel reinforcement area } \\
& \text { C = axial compressive force in concrete } \\
& C_{E} \quad=\text { environmental exposure coefficient } \\
& D \quad=\text { diameter } \\
& D \quad=\text { dead load } \\
& E \quad=\text { Earthquake Load } \\
& E_{c} \quad=\text { modulus of concrete } \\
& E_{f} \quad=\text { modulus of FRP } \\
& E_{S} \quad=\text { modulus of steel } \\
& (E I)_{c r}=\text { Bending stiffness of cracked section } \\
& F \quad=\text { Lateral Fluid Pressure } \\
& H \quad=\text { Soil Load } \\
& L \quad=\text { Live Load } \\
& L_{e} \quad=\text { Active bond length of FRP } \\
& L_{r} \quad=\text { Roof live Load } \\
& M_{n} \quad=\text { Nominal moment capacity } \\
& P_{n} \quad=\text { Nominal axial capacity } \\
& P_{u} \quad=\text { Required axial capacity } \\
& R \quad=\text { Rain Load } \\
& R_{n} \quad=\text { Nominal strength of a member } \\
& R_{S} \quad=\text { Stress Resultant }
\end{aligned}
$$




$$
\begin{array}{ll}
S & =\text { Snow Load } \\
S_{D L} & =\text { Dead load effects } \\
S_{L L} & =\text { Live Load Effects } \\
T & =\text { Creep or shrinkage and temperature load } \\
T_{f} & =\text { Tensile force in FRP } \\
T_{S} & =\text { Tensile force in steel } \\
U & =\text { Required capacity } \\
V_{c} & =\text { Shear capacity of concrete } \\
V_{f} & =\text { Shear capacity of FRP } \\
V_{n} & =\text { Nominal shear capacity } \\
V_{f}^{R} & =\text { Required shear capacity of FRP } \\
V_{S} & =\text { Shear capacity of steel reinforcement } \\
W & =\text { Wind Load }
\end{array}
$$




\section{Chapter 1 Introduction}

Existing concrete structures, built years ago, are experiencing an increase in their service loads. These structures deteriorate with time due to various reasons, the primary one being the new loads exceeding the set design criteria for the structure. Traditional approaches for rehabilitation and retrofitting such as 'exterior bonded steel reinforcement' have been inefficient. (Barbero, 2011) Looking up for newer technologies, Fiber Reinforced Composites (FRP) has emerged as a promising alternative for strengthening of reinforced concrete structures. Following are a few advantages of FRP retrofitting (Barbero, 2011):

- High strength to weight ratio

- Quickly and easily installed

- Corrosive resistant

Following are some of the shortcomings of FRP

- High initial price- Research is on to reduce the initial costs

- Low ductility- American Concrete Institute (ACI) rules are such that the structure does not fail by low ductility.

A reinforced concrete member can be strengthened based on the loading conditions. The following are some of the strengthening methods

- Flexural Strengthening- Bonding FRP material to tension faces of a beam.

- Shear Strengthening- Bonding FRP material inclined or vertically to the beam.

- Axial Confinement- Column is wrapped circumferentially with FRP sheets.

\subsection{Objective}

The objective of this work is to develop a web application that lets the user design the strengthening of Reinforced Concrete (RC) members using composites.

This document discusses the design procedures for strengthening of concrete members. The design procedures described in the book have been further adopted to build the necessary software methods for the web app 'StrengtheningOfRC', the details of which are also provided in this manuscript. Finally, this document also discusses the CADEC front end architecture and data base design.

\subsection{CADEC}

Computer Aided Design Environment for Composites or CADEC is a Web Application built to make composite designing easier, save time and most importantly be accessible to anyone who wants to use this application. It is built based on the book 'Introduction to Composite Materials Design' written by Dr. Ever J. Barbero. The Current version CADEC 14 (CADEC Online, cadec-online.com) supports seven chapters from the book viz., Micromechanics, Ply Mechanics, Macromechanics, Failure, Damage, Textile Reinforced 
Concrete and Thin walled Beams. The present effort aims to add a new module 'Strengthening of Reinforced Concrete Members'. 


\section{Chapter 2 Strengthening of Reinforced Concrete Members}

This chapter is an overview of chapter 'Strengthening of Reinforced Concrete Member' (Barbero, 2011) and what is intended to implement in the Web Application (Saas, 2013).

\subsection{Design of Reliability}

'Design for Reliability' is a probabilistic approach towards finding the value of a property considering the variations involved with it. This design accommodates the variations in the loading conditions, strength of a material used, construction tolerances etc. Material properties that are crucial for any design are obtained by experiments which are variable. Also, loads considered during designing a member have very high probability of variation. For the reasons explained, design for reliability considers a factor $\operatorname{Reliability}\left(R_{e}\right)$. For a normally distributed data of this variable entity, they are represented by a standard deviation $(\varpi)$ and a mean $(\mu)$. The term 'Coefficient of Variation (COV)' is defined for convenience in determining $R_{e}$.

$$
C=\varpi / \mu
$$

The factor $R_{e}$ is defined by $C=\varpi / \mu \quad(2.1)$, where $C_{F}, C_{\sigma}$ are COV of strength and stress respectively.

$$
R_{e}=\frac{1 \pm \sqrt{1-\left(1-\mathrm{z}^{2} \mathrm{C}_{\mathrm{F}}^{2}\right)\left(1-\mathrm{z}^{2} \mathrm{C}_{\sigma}^{2}\right)}}{\left(1-\mathrm{z}^{2} \mathrm{C}_{\mathrm{F}}^{2}\right)}
$$

Designing of FRP strengthening is based on Load and Resistance Factor Design (LFRD) which is based on Design for Reliability. LFRD splits $R$ into two factors; Resistance or Strength Factor $\varphi$ and Load Factor $\alpha$, through which more conservative design is achieved. LFRD $\alpha D L D+\psi j=1 \mathrm{NL} \alpha \mathrm{jLj} \quad$ (2.3) to be satisfied, which states that the strength of the material must be greater than the loads applied after variability factors are introduced. In this equation, permanent loads are represented by Deal Loads $\left(L_{D}\right)$ and other loads like live loads, thermals loads, dynamic loads are represented by $L_{j}$. The combination factor $\psi$ represents that all the other loads are present simultaneously.

$$
\varphi \mathrm{F}>\alpha_{\mathrm{D}} \mathrm{L}_{\mathrm{D}}+\psi \sum_{\mathrm{j}=1}^{\mathrm{N}_{\mathrm{L}}} \alpha_{\mathrm{j}} \mathrm{L}_{\mathrm{j}}
$$

\subsection{FRP Load and Strength Design}

Limit States Design, which is used for FRP Strengthening is derived from LFRD. In this design, strengthening limits for FRP are defined by ultimate limit states and serviceability limit states.

- Ultimate limit states is the condition where a structure is checked under extreme loading combinations that would potentially fail the structure. 
- Serviceability limit states is a performance criterion in which a structure is checked for its service requirements, which is the set of loading combinations experienced by the structure on a regular basis. A structure fails its serviceability due to deformations, vibrations and cracking.

- Strength of RC Member: The nominal capacity of a structure is represented by $F$ which is derived from its material. According to LFRD, strength reduction factor $\varphi$ is introduced to allow variability. The strength reduction factor $\varphi$ accommodates the variability in the material properties and construction tolerances. Hence, strength of RC member is given by the Factored Capacity $\varphi F$. Table 2.1 lists the factors used corresponding RC member type (Barbero, 2011).

Table 2.1- Strength reduction factor $(\varphi)$

\begin{tabular}{|l|c|}
\hline Structural Element & $\varphi$ \\
\hline Beam or slab: Bending or flexure & $0.65-0.9$ \\
\hline Beam shear and torsion & 0.75 \\
\hline Columns with ties & 0.65 \\
\hline Columns with spirals & 0.75 \\
\hline
\end{tabular}

- Required Capacity: A structure bears various types of loads that include permanent loads and live loads. As the design follows LFRD, a loading factor is introduced to accommodate variability of loads. The design considers various types of live loads like, lateral fluid pressure, snow load, rain load, earthquake load, creep or shrinkage and temperature load, soil loads and roof live load. The American Concrete Institute (ACI) recommends the following combinations. Some factors are assigned to a specific combination of loads based on probability of simultaneous occurrence (Barbero, 2011)

$$
\begin{aligned}
& \mathrm{U}=1.2(\mathrm{D}+\mathrm{F}+\mathrm{T})+1.6(\mathrm{~L}+\mathrm{H})+0.5\left(\mathrm{~L}_{\mathrm{r}} \text { or } \mathrm{S} \text { or } \mathrm{R}\right) \\
& \mathrm{U}=1.2 \mathrm{D}+1.6\left(\mathrm{~L}_{\mathrm{r}} \text { or } \mathrm{S} \text { or } \mathrm{R}\right)+(\mathrm{L} \text { or } 0.8 \mathrm{~W}) \\
& \mathrm{U}=1.2 \mathrm{D}+1.6 \mathrm{~W}+0.5\left(\mathrm{~L}_{\mathrm{r}} \text { or } \mathrm{S} \text { or } \mathrm{R}\right) \\
& \mathrm{U}=1.2 \mathrm{D}+\mathrm{E}+\mathrm{L}+0.2 \mathrm{~S} \\
& \mathrm{U}=0.9 \mathrm{D}+1.6 \mathrm{~W}+1.6 \mathrm{H} \\
& \mathrm{U}=0.9 \mathrm{D}+\mathrm{E}+1.6 \mathrm{H}
\end{aligned}
$$

\subsection{Environmental Reduction Factor}

An environmental safety factor is considered for calculating FRP properties like tensile strength, rupture strain and elastic modulus. This is because the environment has an adverse effect on the performance of FRP as it is on the outer surface of the concrete member. The environmental factor depends on the type of fiber. Table 2.2 shows the environmental factors considered in the development of the methods for the software in the present effort. 


$$
\mathrm{f}_{\mathrm{fu}}=\mathrm{C}_{\mathrm{E}} \mathrm{f}_{\mathrm{fu}}^{*}
$$

Table 2.2- Environmental Reduction Factor (Barbero, 2011)

\begin{tabular}{|c|c|c|}
\hline Exposure Conditions & Fiber Type & $\begin{array}{c}\text { Environmental reduction } \\
\text { factor } C_{F}\end{array}$ \\
\hline \multirow{3}{*}{ Interior exposure } & Carbon & 0.95 \\
\hline & Glass & 0.75 \\
\hline & Aramid & 0.85 \\
\hline \multirow{3}{*}{$\begin{array}{c}\text { Exterior exposure (bridges, } \\
\text { piers, and unenclosed parking } \\
\text { garages) }\end{array}$} & Carbon & 0.85 \\
\hline & Glass & 0.65 \\
\hline & Aramid & 0.75 \\
\hline \multirow{3}{*}{$\begin{array}{l}\text { Aggressive environment } \\
\text { (chemical plants and } \\
\text { wastewater treatment plants) }\end{array}$} & Carbon & 0.85 \\
\hline & Glass & 0.50 \\
\hline & Aramid & 0.70 \\
\hline
\end{tabular}

The rupture strain is adjusted using

$$
\varepsilon_{f u}=C_{E} \varepsilon_{f u}^{*}
$$

The behavior of unidirectional FRP behaves linear elastically, and therefore Hooke's law can be used to determine the modulus for FRP as in

$$
E_{f u}=\frac{F_{E}}{\epsilon_{f u}}
$$

\subsection{Flexure Strengthening}

\subsubsection{Failure modes}

An FRP Strengthened RC beam could fail in several failure modes. FRP design procedure is checked for following prominent failure modes.

- Crushing of concrete due to compression before steel reinforcement start yielding.

- Rupture of FRP after steel reinforcement yields in tension.

- Yielding of steel reinforcement in tension followed by crushing of concrete.

- Delamination of concrete cover by shear or tension.

- Debonding of FRP from concrete surface.

Crushing of concrete is assumed to occur when the compression strain reaches a value of 0.003. The rupture of the FRP is assumed to occur when the strain reaches the designed rupture strain. The most concerning issue is crack-induced debonding. To prevent this failure mode, the effective strain of the FRP needs to be limited to the strain value. Also, a strength reduction factor for the FRP is introduced to address issues of reliability of the FRP. 


\subsubsection{Stress and Stain Levels in FRP Reinforcement}

The stress and strain levels are important to determine and compare to the ultimate limit states. The maximum strain is achieved at one of three situations,

1) The strain level of the FRP when the concrete crushes,

2) When the FRP ruptures, or

3) When the FRP reinforcement debonds from the concrete. The effective strain can be calculated using following equation

$$
\varepsilon_{\mathrm{fe}}=\varepsilon_{\mathrm{cu}} \frac{\mathrm{d}_{\mathrm{f}}-\mathrm{c}}{\mathrm{c}}-\varepsilon_{\mathrm{bi}} \leq \varepsilon_{\mathrm{fd}}
$$

\subsubsection{Strength Reduction Factor}

The application of external FRP reinforcement does affect the ductility of the concrete beam and though loss is sometimes negligible, the situation of large losses must be addressed. The tension controlled state must be checked with accordance with $\varphi=\left\{\begin{array}{c}0.9 \text { for } \varepsilon_{\mathrm{t}} \geq 0.005 \\ 0.65+\frac{0.25\left(\varepsilon_{\mathrm{t}}-\varepsilon_{\mathrm{sy}}\right)}{0.005-\varepsilon_{\mathrm{sy}}} \text { for } \varepsilon_{\mathrm{sy}}<\varepsilon_{\mathrm{t}}<0.005 \quad \text { (2.14) which has the strength reduction } \\ 0.65 \text { for } \varepsilon_{\mathrm{t}} \leq \varepsilon_{\mathrm{sy}}\end{array}\right.$ factor for ductile sections as 0.9 and for brittle sections as 0.65 with a linear transition for steel that has not yielded (Barbero, 2011)

$$
\varphi=\left\{\begin{array}{c}
0.9 \text { for } \varepsilon_{\mathrm{t}} \geq 0.005 \\
0.65+\frac{0.25\left(\varepsilon_{\mathrm{t}}-\varepsilon_{\mathrm{sy}}\right)}{0.005-\varepsilon_{\mathrm{sy}}} \text { for } \varepsilon_{\mathrm{sy}}<\varepsilon_{\mathrm{t}}<0.005 \\
0.65 \text { for } \varepsilon_{\mathrm{t}} \leq \varepsilon_{\mathrm{sy}}
\end{array}\right.
$$

\subsubsection{Serviceability}

To prevent excessive deformations of the reinforced concrete section with FRP reinforcement, the reinforcing steel should be prevented from yielding under services loads. The stress in the steel reinforcement under service load should be limited to $80 \%$ of the yield strength as shown in $\mathrm{f}_{\mathrm{s}, \mathrm{s}} \leq 0.8 \mathrm{f}_{\mathrm{y}} \quad$ (2.15). In addition, the compressive stress in concrete under service load should be limited to $45 \%$ of the compressive strength, as shown in $\mathrm{f}_{\mathrm{c}, \mathrm{s}} \leq$ $0.65 f_{c}^{\prime}$

$$
\begin{aligned}
& \text { (2.16) } \\
& (\text { Barbero, 2011) (Saas, 2013) } \\
& \mathrm{f}_{\mathrm{s}, \mathrm{s}} \leq 0.8 \mathrm{f}_{\mathrm{y}} \\
& \mathrm{f}_{\mathrm{c}, \mathrm{s}} \leq 0.65 \mathrm{f}_{\mathrm{c}}^{\prime}
\end{aligned}
$$

\subsubsection{Nominal Flexure Strength}

The nominal flexure strength of a reinforced concrete section with external FRP

reinforcement is equated using $M_{n}=A_{s} f_{s}\left(d \frac{\beta_{1} c}{2}\right)+\psi_{f} A_{f} f_{f e}\left(h \frac{\beta_{1} c}{2}\right) \quad$ (2.17). The reduction factor for FRP is applied with the recommended value of 0.85 (ACI 440.2R-08, 2008)

$$
M_{n}=A_{s} f_{s}\left(d \frac{\beta_{1} c}{2}\right)+\psi_{f} A_{f} f_{f e}\left(h \frac{\beta_{1} c}{2}\right)
$$

2.4.6 Unstrengthened Behavior 
An unstrengthened reinforced concrete member is a member without FRP. The behavior of the unstrengthened section of reinforced concrete is almost linear. During initial loading, flexure on the tension surface of the concrete begin to develop when the stress reaches the tension strength of the concrete. Once cracking occurs, the tensile force is then transferred to the steel. Once the steel stress has reached the yield strength, the reinforced concrete structure will redistribute the forces. If the steel yields before the concrete crushes it responds in a ductile manner. The cracking patterns in the concrete become evident, and the beam experiences considerable deflections before failure. If the reinforced concrete member has a significant amount of steel reinforcement, therefore requiring more loading to yield, the concrete will crush before yielding of the steel occurs, causing failure. This is known as brittle failure (Barbero, 2011)

\subsubsection{Strengthened Behavior}

When FRP is applied, the reinforced concrete member is considered to be strengthened. At first, the stiffness of the member is basically unchanged when compared to the unstrengthened behavior. This is because the applied FRP strips are very thin and do not cause any notable changes in the stiffness of the structure, but the behavior is affected. The tension force is shared between the steel and FRP reinforcement, after the concrete has cracked. The FRP contribution for tension force now becomes more relevant since it is located furthest from the neutral axis. After the steel yields, the FRP is forced to carry the load the steel was carrying. The strengthened reinforced concrete member collapses at higher levels because of the FRP but observes lowers deflections compared to the unstrengthened member, making the member less ductile.

In addition to the steel and FRP yielding before the concrete crushes, strengthened reinforced concrete members may also be affected by other failure modes than just large loads causing. The FRP is capable of failing due to its delamination from the concrete (Barbero, 2011)

\subsubsection{Strengthening Configuration}

Because of the limitations of concrete, steel reinforcement, and FRP, the failure mode of the system needs to be determined. The first possible failure mode for the strengthened reinforced concrete member is the FRP failing before or after the steel reinforcement yields but prior to the concrete crushing. This failure mode is known as weak strengthening configuration (WSC). The other failure mode that needs to be considered is when the concrete crushes on the compression side of the member before or after the yielding of the steel reinforcement, this is called strong strengthening configuration (SSC). Also, there is a stage known as the balanced strengthening configuration (BSC) which is used for preliminary design purposes. The BSC case is when the failure of the concrete and the FRP occurs simultaneously and can be either elastic or inelastic depending on whether the steel has yielded or not.

\subsubsection{Design procedure for Flexure strengthening}


The design procedure for FRP Strengthening of beam in flexure is followed in steps as described below:

Step 1- The first step is to determine the initial moment capacity of the beam and compare it with minimum required capacity. Also, the maximum allowable reinforcement of the original section is calculated.

Step 2- The initial strain as well as the stress distribution of the soffit beam is calculated while only considering dead loads. Next, the FRP system is selected and the preliminary values are calculated using the environmental reduction factor.

Step 3- Having the original capacity and the initial strain, an approximate area of FRP can be calculated. With an estimate of the area of FRP, the actual area of FRP to be applied and the corresponding configuration is determined. Next, the capacity of the strengthened system is determined and whether it meets the required capacity or not is verified. If it does not, an increase the area of FRP until the strengthened beam satisfies the required capacity is recommended. Lastly, the serviceability requirements must be verified.

\subsection{Shear Strengthening}

RC Member can be further strengthened in shear to prevent failure from shear cracking. For shear strengthening, FRP is applied transversely to the cross section. FRP is then wrapped around the beam in a continuous or a discontinuous way. To prevent debonding or delamination of FRP from the concrete surface, shear strain limits are used (ACI 440.2R-08, 2008)

\subsubsection{Completely Wrapped members}

A reinforced concrete member can be wrapped completely; therefore all the sides of the section have FRP reinforcement applied externally around them. This wrapping scheme however, is known to produce a failure mode where there is a loss of aggregate interlock of the concrete before the ultimate fiber strain is achieved. To prevent this failure mode, the maximum strain of the design is limited to $0.4 \%$ when members are completely wrapped $\epsilon_{\mathrm{fe}}=0.004 \leq$ $0.75 \epsilon_{\mathrm{fu}}$

(2.18). Higher strains should not be used for FRP shear strengthening (ACI 440.2R-08, 2008)

$$
\epsilon_{\mathrm{fe}}=0.004 \leq 0.75 \epsilon_{\mathrm{fu}}
$$

\subsubsection{Shear Spacing}

The spacing limitations for FRP strips for shear strengthening adhere to the limits as prescribed by ACI for internal steel shear reinforcement. This states "for non-prestressed members the spacing shall not exceed half of the depth or 24 inches $(610 \mathrm{~mm})$ )" The spacing is from the centerline of one FRP strip to the next (ACI 440.2R-08, 2008).

\subsubsection{Reinforcement Limits}


The sum of the contributed strength of the FRP reinforcement and steel shear reinforcement is taken as the total shear strength. The total shear reinforcement is limited based on the ACI 318 article $\mathrm{V}_{\mathrm{v}}+\mathrm{V}_{\mathrm{f}} \leq 0.66 \sqrt{\mathrm{f}_{\mathrm{c}}^{\prime}} \mathrm{bc} \quad$ (2.19) for steel which gives the shear limit.

$$
\mathrm{V}_{\mathrm{v}}+\mathrm{V}_{\mathrm{f}} \leq 0.66 \sqrt{\mathrm{f}_{\mathrm{c}}^{\prime}} \mathrm{bc}
$$

\subsubsection{Discontinuous Wrapping}

The shear capacity of the steel reinforcement of the strengthened section when the FRP is stripped along the length of the beam, in intermittent distances, can be determined using

$V_{s}=f_{y} A_{s v} \frac{d}{s_{s}}[\sin \alpha+\cos \alpha]$ (2.20). It is a function of the distance between reinforcement and the angle of the steel reinforcement (Barbero, 2011)

$$
\mathrm{V}_{\mathrm{s}}=\mathrm{f}_{\mathrm{y}} \mathrm{A}_{\mathrm{sv}} \frac{\mathrm{d}}{\mathrm{s}_{\mathrm{s}}}[\sin \alpha+\cos \alpha]
$$

The FRP strip's shear capacity can be calculated using $V_{f}=f_{f e}\left(2 n_{f} t_{f} w_{f}\right) \frac{d_{f v}}{s_{s}}\left[\sin \alpha_{f}+\right.$ $\left.\cos \alpha_{\mathrm{f}}\right](2.21)$ which also a function of the applied angle of the FRP, as well as the distance of the strips.

$$
V_{f}=f_{f e}\left(2 n_{f} t_{f} w_{f}\right) \frac{d_{f v}}{s_{s}}\left[\sin \alpha_{f}+\cos \alpha_{f}\right]
$$

\subsubsection{Continuous Wrapping}

When the applied FRP reinforcement is layered along the length of the beam, the applied angles of the steel and FRP reinforcement are assumed to be $90^{\circ}$. Therefore the shear steel capacity is calculated by $V_{s}=f_{v} A_{s v} \frac{d}{s_{s}} \quad$ (2.22) and the FRP capacity is calculated

$$
\begin{gathered}
\operatorname{using} \mathrm{V}_{\mathrm{f}}=\mathrm{f}_{\mathrm{fe}}\left(2 \mathrm{n}_{\mathrm{f}} \mathrm{t}_{\mathrm{f}} \mathrm{W}_{\mathrm{f}}\right) \mathrm{d}_{\mathrm{fv}} \quad \text { (2.23) (Barbero, 2011) } \\
\mathrm{V}_{\mathrm{s}}=\mathrm{f}_{\mathrm{v}} \mathrm{A}_{\mathrm{sv}} \frac{\mathrm{d}}{\mathrm{s}_{\mathrm{s}}} \\
\mathrm{V}_{\mathrm{f}}=\mathrm{f}_{\mathrm{fe}}\left(2 \mathrm{n}_{\mathrm{f}} \mathrm{t}_{\mathrm{f}} \mathrm{w}_{\mathrm{f}}\right) \mathrm{d}_{\mathrm{fv}}
\end{gathered}
$$

\subsubsection{Design procedure for shear strengthening}

In order to determine the number of layers needed to meet the required shear capacity of the beam, the initial shear capacity of the beam must be ascertained. That is, when the beam is unstrengthened, all the shear capacity comes from the concrete and steel stirrups. The initial capacity is then compared to the minimum required strength and the maximum allowable strengthening governed by ACI (Barbero, 2011). With initial capacities, an estimate of the number of layers is obtained based on the design scheme, continuous, discontinuous, complete wrapping, three-sided, or two-sided. By rearranging $V_{s}=f_{v} A_{s v} \frac{d}{s_{s}} \quad$ (2.22) and $V_{f}=$ $\mathrm{f}_{\mathrm{fe}}\left(2 \mathrm{n}_{\mathrm{f}} \mathrm{t}_{\mathrm{f}} \mathrm{w}_{\mathrm{f}}\right) \mathrm{d}_{\mathrm{fv}} \quad(2.23)$, the number of layers can be determined as seen in $\mathrm{n}_{\mathrm{f}}=$ 
$\frac{1}{\psi_{\mathrm{f}}}\left[\frac{\mathrm{V}_{\mathrm{u}}}{\varphi}-\mathrm{V}_{\mathrm{c}}-\mathrm{V}_{\mathrm{s}}\right] \frac{1}{\mathrm{f}_{\mathrm{fe}}\left(2 \mathrm{t}_{\mathrm{f}} \mathrm{w}_{\mathrm{f}}\right) \mathrm{d}_{\mathrm{fv}}}(2.24)$ where the nominal capacity is replaced by the required capacity.

$$
\mathrm{n}_{\mathrm{f}}=\frac{1}{\Psi_{\mathrm{f}}}\left[\frac{\mathrm{V}_{\mathrm{u}}}{\varphi}-\mathrm{V}_{\mathrm{c}}-\mathrm{V}_{\mathrm{s}}\right] \frac{1}{\mathrm{f}_{\mathrm{fe}}\left(2 \mathrm{t}_{\mathrm{f}} \mathrm{w}_{\mathrm{f}}\right) \mathrm{d}_{\mathrm{fv}}}
$$

After determining the number of layers to be applied to the reinforced concrete beam, the shear capacity of the FRP is calculated and the limit for the total shear reinforcement is verified (Barbero, 2011) 


\section{Chapter 3 The Microsoft .NET Framework}

The .NET Framework is an environment from Microsoft for developing and executing software applications. It provides rich 'standard runtime services' for programs written in a variety of languages. Figure 3-1 shows the .NET framework components in a stack.

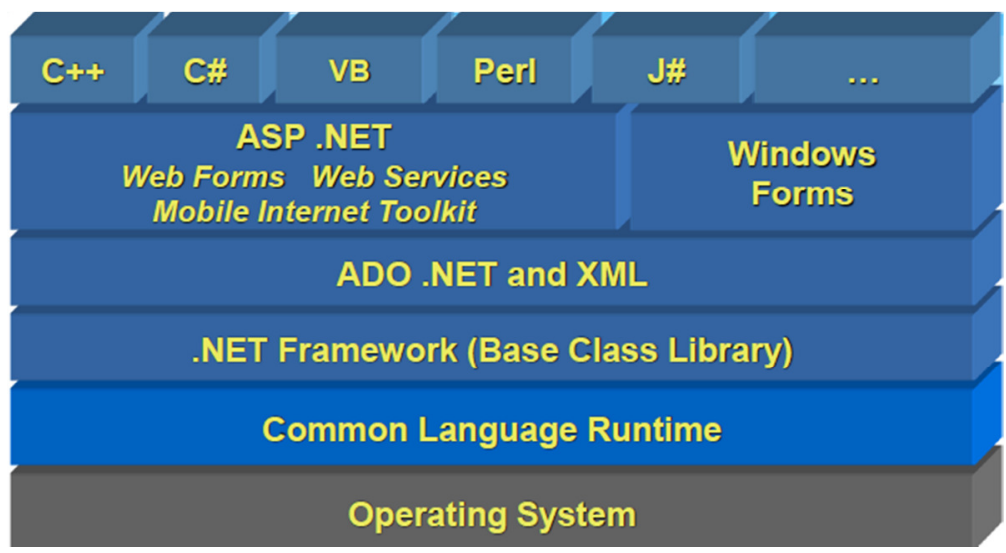

Figure 3-1: .NET Framework Stack

\subsection{Elements of Framework}

3.1.1 The Common Language Runtime (CLR): CLR is a part of the .NET framework that is built on the operating system. It manages the execution of programs written in different supported languages. During executions it manages memory allocation, thread execution, garbage collection, exception handling, code safety verifications and other system services on all supported languages. During runtime, CLR also converts .NET compiled code into native code which is understood by operating system. For all its functionalities, CLR is often referred as Managed Execution Environment.

3.1.2 The Base Class Library (BCL): The .NET framework Base Class library is a library of classes written in the $\mathrm{C \#}$ language. It provides core functionalities for the .NET Framework architecture. It is built on top of CLR, implying it uses the functionalities present in CLR. It is the foundation on which .NET applications, components and controls are built. A component or software component is a software element that can be independently deployed. The .NET Framework class libraries are organized in a hierarchical tree structure and each class is arranged in a Namespace that best qualifies its purpose. Namespace in .NET is a logical grouping of classes for an intuitive identification of purpose. For example, the namespace 'System' is the root for all the classes in the .NET framework. It provides access to system services. It consists of classes like Collections, I/O, Configuration, Serialization, Diagnostics and many more fundamental classes. BCL is shared across all the languages supported by .NET. 
3.1.3 The Framework class Library (FCL): .NET framework class library consists of classes, interfaces and types that can be used in a program. It includes many prominent .NET libraries like ASP.NET, ADO.NET, Windows Forms, GUI development.

\subsection{ADO.NET}

DataProviders are components that enable access to data sources (databases).ActiveX Database Objects (ADO.NET) is a data provider which is a gateway to data bases in .NET framework. It is a set of classes in the form of class libraries that facilitate data access for 'managed languages'. Managed languages are those programming languages that are supported by the .NET framework. ADO.NET provides a comprehensive and complete set of libraries for data access. This data access service is independent of the database being used. Thus, an application can access data from multiple databases using just one technology, ADO.NET. It is also interesting to note that, ADO.NET can be used for the development of Web applications as well as Windows applications.

The application developed for implementing the chapter 'Strengthening of Reinforced Concrete using FRP' uses the SQL Server 2008 database. The SQL server data provider component 'System.Data.SqlClient' is used to enable communication with the database.

\subsection{ASP.NET}

Active server pages (ASP) was the first version of the Web Development tool developed by Microsoft. ASP.NET evolved from the basic ASP, which is considered to be a simple language. It's usage in web development has seen a sustained increase, while application development itself has seen new dimensions with technology growth.

ASP.NET enables the creation of web sites and XML Web Services and is a core element of the .NET Framework. It is based on an extensible component- based architecture, meaning the architechture allows the developer to build new components. It is more powerful than its predecessor ASP. ASP.NET contains page and control framework that is used by developers to create the user interface(UI) of the Web applications. This framework is called Web Forms as in Figure 3-1. Server controls or web form controls are the essence of Web Forms. These controls provide mechanism for reuse and encapsulation that make rapid application development. Server controls gave rise to new set of developers called component developers who can add to the set of built-in ASP.NET server controls by implementing new custom controls. ASP.NET is implemented in System.Web namespace and various sub namespaces, collectively represent the features used in developing Web applications and XML Web Services. 


\section{Chapter 4 : Project Kernel}

As the name indicates it is the core of the Web Application, CADEC (CADEC Online, cadec-online.com). The Kernel is a C\# Class Library built with three types of classes.

- Type 1: Are business objects that do all the calculations involved in designing FRP for different types of Reinforced Concrete Members (Chapter 2). Design software methods for strengthening of RC member developed by (Saas, 2013) are included in this project.

- Type 2: Consists of classes that communicate with the database and methods to execute stored procedures up on request from the user.

- Type 3: Defines the elements of the chapter viz., properties of concrete member, FRP, Load Configuration.

The following sections talks in detail about the classes in Kernel. In software development terminology Business Logic layer is the middle layer of a 3-tier application. In this application the 'Kernel' is the business logic layer.

\subsection{Design Software Methods}

Software methods for executing the design procedures in this application have been adopted from (Saas, 2013). These software methods are written in C\#, an object oriented language supported by the .NET framework on which the CADEC application is built-on. They are implemented and tested on a simple Windows Forms application. The following section discusses the software methods and their implementation in the Web Application.

The intent behind the development of the software methods (classes) by (Saas, 2013) has been maintained while implementing them in the Web Application. They can accommodate new methods in the class or new classes can be added to the project. In other words these methods or calculations are functions that are called during the design process. The design process is executed and encapsulated in the WebReusables project. Figure 4-1 shows the class hierarchy of software methods in Kernel. 


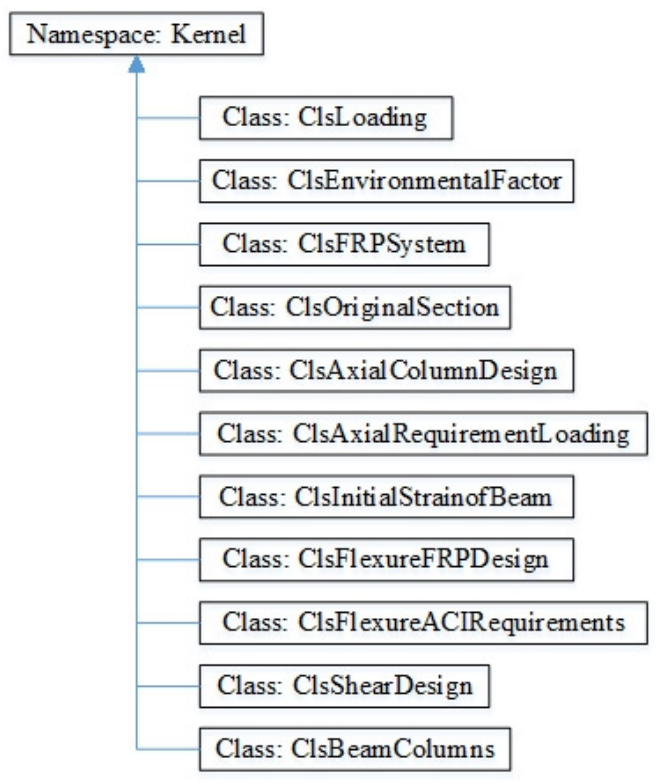

Figure 4-1: Software methods in Kernel namespace

\subsubsection{Class: ClsLoading}

The class diagram of ClsLoading Figure 4-2 consists of only one method, MaximumLoadingScenario. This method calculates maximum required strength of a $\mathrm{RC}$ member using ACI uidelinesg. Maximum required strength is required during the designing of FRP in flexure, shear and Axial loading state.

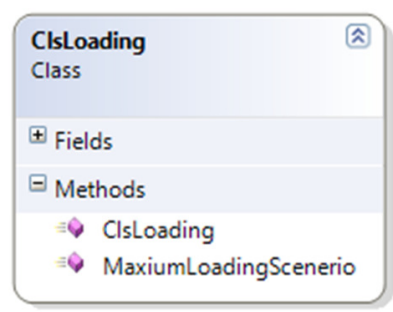

Figure 4-2: Class Diagram- ClsLoading 


\subsubsection{Class: ClsFRPEnvironmentalFactor}

ACI guidelines recommend usage of strength reduction factor for the FRP due to its exposure to environment. The reduction factor is independent of the loading state. EnvironmentalFactor method in Figure 4-3 considers interior, exterior and aggressive types of environments. The method considers only carbon, glass and aramid types fiber for calculation of the reduction factor.

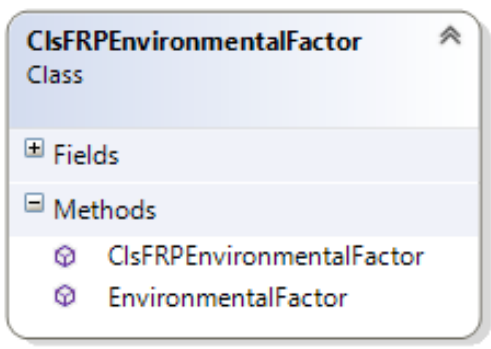

Figure 4-3: Class Diagram- ClsFRPEnvironmentalFactor

Certain corrections have been made in the EnvironmentalFactor method from the code adopted from (Saas, 2013), these are explained in detail in the appendix.

\subsubsection{Class: ClsFRPSystem}

As the name indicates ClsFRPSystem is made up of methods shown in Figure 4-4 that calculate the necessary FRP properties. Design procedures require additional properties like Modulus of FRP, Ultimate Rupture Strain of FRP and Ultimate Tensile Strength of FRP. Methods in this class find use during the strengthening of RC beam in flexure.

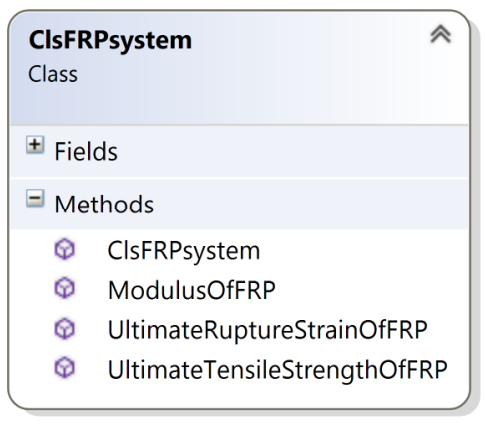

Figure 4-4: Class Diagram- ClsFRPSystem

\subsubsection{Class: ClsOriginalSection}

Concrete members to be strengthened by FRP have to go through initial analysis based on ACI requirements. 'ClsOriginalSection' (Figure 4-5) constitutes methods to conduct initial checks of beam in flexure, shear and for the column in axial loading. A member has to be checked for minimum loading requirement, nominal capacity of the member, maximum allowable strengthening and actual amount of strengthening. (Section 2.2 FRP Load and Strength Design) 


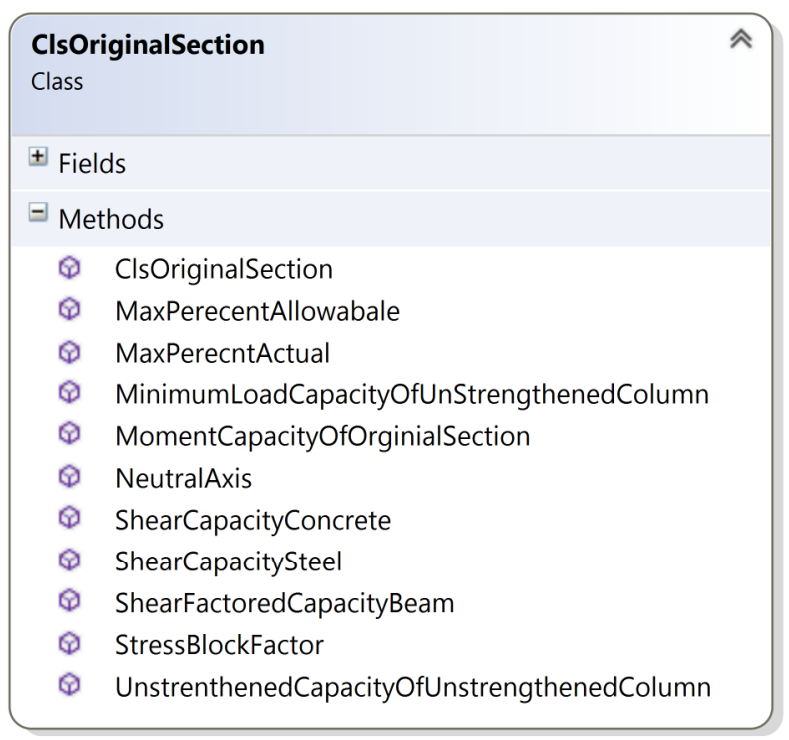

Figure 4-5: Class- ClsOriginalSection

\subsubsection{Class: ClsAxialColumnDesign}

ClsAxialColumnDesign (Figure 4-6) is built with methods that aid in strengthening of concrete column under axial loading. Methods in this class include:

- FRPEfficiencyFactor calculates a factor depending on cross section of column to determine confined pressure of the member.

- Confined pressure imposed by FRP on column by wrapping is calculated by ConfinedPressure.

- Number of Layers of FRP required to withstand confined pressure is calculated by RequiredNumberOfLayers.

- FactoredAxialCapacity calculates factored capacity of confined column after determining actual strength of confined member (Section Error! Reference source not found.)

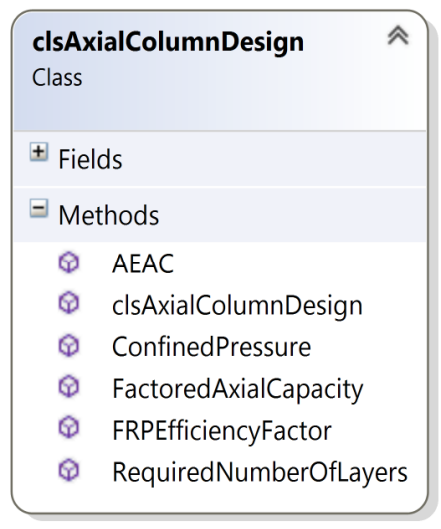

Figure 4-6: Class- ClsAxialColumnDesign 


\subsubsection{Class: ClsAxialColumnACIRequirements}

ClsAxialColumnACIRequirements (Figure 4-7) is built with methods that check whether the design of the axial column strengthening meets ACI requirements.

The methods calculate confinement ratio, compressive strain, and concrete, steel stresses. These methods implement equations as discussed in (Section Error! Reference source not found.)

ClsAxialColumnACIRequirements
Class
$\square$ Fields
Methods
$\ominus$ ClsAxialColumnACIRequirements
$\ominus$ CompressiveStrainOnConfinedColumn
$\ominus$ ConfinementRatio
$\ominus$ SteelStress

Figure 4-7: Class- ClsAxialColumnACIRequirements

\subsubsection{Class: ClsInitialStrainOfBeam}

ClsInitialStrainOfBeam (Figure 4-8) has methods to calculate initial conditions in the RC beam before strengthening.

- NewNeutralAxis calculates the neutral axis location after cracks appear in a beam.

- EffectiveStiffnessOfCrackedBeam calculates stiffness of the beam after cracks appear and before FRP strengthening

- InitialStrain calculates strain due to loading before it is analyzed for strengthening, which is required in the calculation of required capacity.

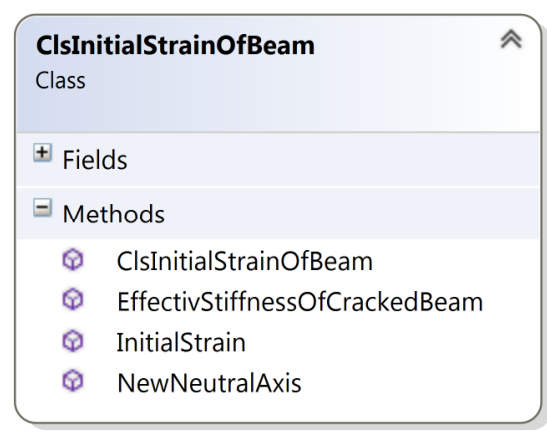

Figure 4-8: Class- ClsInitialStrainOfBeam 


\subsubsection{Class: Class- ClsFlexureFRPDesign}

\begin{tabular}{|c|c|c|}
\hline \multicolumn{2}{|c|}{$\begin{array}{l}\text { ClsFlexureFRPDesign } \\
\text { Class }\end{array}$} & 令 \\
\hline \multicolumn{3}{|c|}{ \pm Fields } \\
\hline \multicolumn{3}{|c|}{$\exists$ Methods } \\
\hline$\varphi$ & AreaFRPforBSC & \\
\hline$\varphi$ & ClsFlexureFRPDesign & \\
\hline$\varphi$ & NeutralAxisSSCSteelNotYeilding & \\
\hline$\varphi$ & NeutralAxisSSCSteelYeilding & \\
\hline$\ominus$ & NeutralAxisWSCSteelNotYeild & \\
\hline$\varphi$ & NeutralAxisWSCSteelYeild & \\
\hline$\varphi$ & NominalMomentCapacityWSCSteelNotYeild & \\
\hline$\oplus$ & NominalMomentCapaictySSCSteelNotYeild & \\
\hline$\varphi$ & NominalMomentCapaictySSCSteelYeild & \\
\hline$\varphi$ & NominalMomentCapcityWSCSteelYeild & \\
\hline$\oplus$ & NumberOfLayers & \\
\hline$\varphi$ & StrainCompression & \\
\hline$\varphi$ & StrainEffective & \\
\hline$\ominus$ & StrainFRPSSC & \\
\hline$\varphi$ & StrainSteelTensileSSC & \\
\hline$\varphi$ & StrainSteelTensileWSC & \\
\hline
\end{tabular}

Figure 4-9: Class- ClsFlexureFRPDesign

The design process for strengthening an $\mathrm{RC}$ member in flexure first checks for the initial ACI conditions, then calculates the number of layers required to meet the loading capacity required in the Balanced Service Conditions (BSC) (Barbero, 2011). Depending on the user input of FRP layers, application further calculates if user input makes it a weak strengthening configuration or strong strengthening configuration. ClsFlexureFRPDesign (Figure 4-9) includes all the methods involved in the strengthening of beam in flexure. It includes initial checks and serviceability checks.

\subsubsection{Class: Class- ClsFlexureACIRequirements}

\begin{tabular}{|l} 
ClsFlexureACIRequirements \\
Class \\
$\square$ Methods \\
$\ominus$ ClsFlexureACIRequirements \\
$\ominus$ NeutralAxisStageB \\
$\ominus$ StressConcrete \\
$\ominus$ StressFRP \\
$\ominus$ StressSteel \\
\hline
\end{tabular}

Figure 4-10: Class- ClsFlexureACIRequirements 
Strengthening of beam in flexure has to abide by the ACI serviceability conditions. ClsFlexureACIRequirements (Figure 4-10) contains methods that check if FRP strengthening design stands good for the ACI requirements. These methods check for stress levels in FRP, steel and concrete before and after strengthening (Section 2.4 Flexure Strengthening).

\subsubsection{Class: Class- ClsShearDesign}

\begin{tabular}{|c|c|c|}
\hline \multicolumn{2}{|c|}{$\begin{array}{l}\text { clsShearDesign } \\
\text { Class }\end{array}$} & 令 \\
\hline \multicolumn{3}{|c|}{$\nabla$ Methods } \\
\hline$\theta$ & BondReductionFactor & \\
\hline$\theta$ & clsShearDesign & \\
\hline$\varphi$ & MaxAllowableShearReinforcement & \\
\hline$\varphi$ & MaxShearSpacingFRP & \\
\hline$\varphi$ & NumberOfLayersContinouslyWrapped & \\
\hline$\theta$ & NumberOfLayersDiscontinousWrapping & \\
\hline$\varphi$ & RequiredShearCapacilyFRP & \\
\hline$\varphi$ & ShearCapacityFRPContinousWrapping & \\
\hline$\varphi$ & ShearCapacityFRPDiscontinousWrapping & \\
\hline$\varphi$ & ShearReinforcement & \\
\hline$\theta$ & StrainEffective & \\
\hline
\end{tabular}

Figure 4-11: ClsShearDesign

ClsShearDesign (Figure 4-11) class is a package of methods that will find use during every stage of designing FRP strengthening of concrete beam member in shear. The methods include initial checks and serviceability checks which complete the design of strengthening of beam in shear

\subsection{Database Design}

As mentioned earlier, this application is built with intent to add the Chapter'Strengthening of Reinforced concrete' to CADEC Architecture. Microsoft SQL Server 2008 database software is chosen to maintain software integrity with CADEC 14. Database schema of CADEC 14 is an exhaustive design to accommodate composite material properties. But the chapter 'Strengthening of Reinforced Concrete' demands new database objects because of the fact that - while most of the chapters deal with calculating composite material properties, this chapter is a step forward from rest of the book. It deals with designing of FRP which depends on the concrete member condition.

New database schema is designed and created in consent with CADEC database conventions (Cosso, 2011). The Relational Database Management (RDBMS) concepts are used in design. Requirements are consolidated to create four database entities CADEC_RCMemberConfiguration (Figure 4-12), CADEC_RCMemberLoad (Figure 4-13), CADEC_FRP (Figure 4-14) and CADEC_BeamColumnSteelProperties (Figure 4-12). The database created is independent of the existing CADEC 14 database. 
The created stored procedures perform data manipulation activities like insert, update, delete and retrieve data from the aforementioned tables. The stored procedures are called upon by the user actions from the webpage.

SQL Server 2008 Express Edition is used to establish the database. The Express Edition is machine specific, which means it is not accessible from machines other than the one on which it is installed. In order to add the database objects in to CADEC database, SQL scripts that create them (Appendix ) must be executed in the CADEC Sever. The following section discusses the tables and the relations between them.

\subsubsection{Tables and Procedures}

Table-CADEC_RCMemberProperties: In this context, a concrete member is defined by its geometric, steel reinforcement and concrete properties. CADEC_RCMemberProperties is designed to define any type of concrete member. Some of the fields in the table are only specific to a member type. The kernel program writes null values into those fields for the other types, while the web program shows only relevant properties to the user. Each record is then identified by a unique Id, auto-generated by the database while inserting a new record.

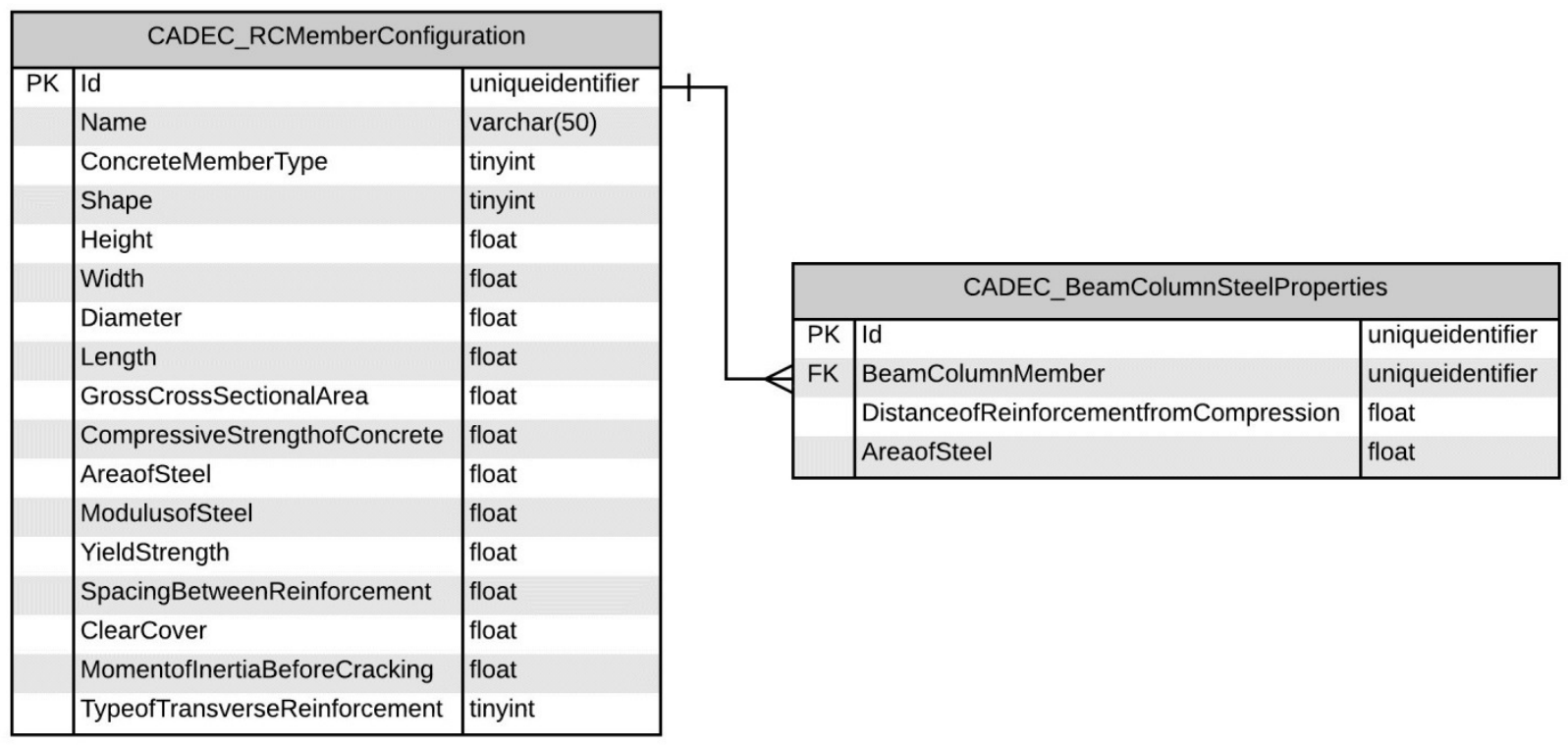

Figure 4-12: Table schema of CADEC_RCMemberProperties, CADEC_BeamColumnSteelProperties and their foreign key relation

Table-CADEC_BeamColumnSteelProperties: The design procedure to strengthen the beamcolumn RC member considers individual steel reinforcement properties (Error! Reference source not found.). Hence, there arose a need to create a new table CADEC_BeamColumnSteelProperties. Each record in this table is identified by a unique id and the RC Member to which the steel reinforcement belongs to. (Figure 4-12) shows foreign key relation between entities CADEC_BeamColumnSteelProperties and CADEC_RCMemberProperties. 
Table-CADEC_RCMemberLoads: Design procedures consider 13 types of loads to calculate maximum required strength of an $\mathrm{RC}$ member in Flexure, shear and axial loading state. The factored moment and the factored axial loads are required to calculate the maximum required load for Beam Column member. (Figure 4-13) shows the schema of CADEC_RCMemberLoads. Each record in the table defines the loading state of a member. It allows the user to store any number of load combinations in the database and can identify a record by its name.

\begin{tabular}{|c|c|c|}
\hline \multicolumn{3}{|c|}{ CADEC_RCMemberLoads } \\
\hline PK & Id & uniqueidentifier \\
\hline & Name & varchar(50) \\
\hline & DeadLoad & float \\
\hline & LiveLoad & float \\
\hline & LateralFluidPressureLoad & float \\
\hline & Snowload & float \\
\hline & RainLoad & float \\
\hline & WindLoad & float \\
\hline & EarthquakeLoad & float \\
\hline & TemparatureCreepShrinkageLoad & float \\
\hline & SoilLoad & float \\
\hline & RoofLiveLoad & float \\
\hline & FactoredMomentLoading & float \\
\hline & FactoredAxialLoading & float \\
\hline & ExposureCondition & tinyint \\
\hline
\end{tabular}

Figure 4-13: Table Schema- CADEC_RCMemberLoads

Table-CADEC_FRP: CADEC 14 calculates the composite's properties like tensile strength and ultimate strain but does not allow the user to store these values. CADEC_FRP is a database entity to store the FRP properties required during the calculations.

\begin{tabular}{|l|l|l|}
\hline \multicolumn{2}{|c|}{ CADEC_FRP } \\
\hline PK & Id & uniqueidentifier \\
& Name & varchar(50) \\
& TensileStrengthofFRP & float \\
& PlyThickness & float \\
& UltimateStrain & float \\
& NumberofLayers & int \\
& FiberType & tinyint \\
\hline
\end{tabular}

Figure 4-14: Table Schema- CADEC_FRP

Stored Procedures: A stored procedure is a set of SQL Statements or simply a Structured Query language (SQL) function with a name, which can be stored in a database in a compiled form. Stored procedures improve productivity because statements need to be written only once. They are also helpful in regulating user access. Communicating with the database through the stored procedures prevents the user from modifying the database.

In this application basic CRUD (Create, Retrieve, Update and Delete) operations on above database entities are executed through procedures. The downside of stored procedures is that, a 
single procedure can do only one basic operation. It leads to a minimum of four procedures for each table. Thus, each type of data retrieval query would need a unique procedure to execute. List of stored procedures is included in appendix (Appendix ). 


\section{Chapter 5 Project 'Web'}

The project 'Web' could be termed as the presentation layer of the web application that has been developed in the present effort. This project consists of webpages that are accessible to the user. Webpages are a gateway into the application for any Web Application. The styles for presentation viz. font size, style, background color for rows etc., have been inherited from CADEC 14. Also, webpages have been organized in a way similar to that of CADEC 14. They are organized into two sections; one being the design section termed as 'Chapters' and other being 'My Documents' section. The 'Chapters' section consists of the webpages that facilitate the user in the FRP Strengthening of an RC Member. The webpages in the 'My Documents' section allow user to store relevant design data to be saved into the database which can be retrieved during the designing process. The following section explains how the designing process has been presented in the webpages.

\subsection{My Documents}

Webpages in this section provide services like storing new data, updating and deleting existing data from the database depending on the choice of the user. Here, rows that appear on the web page are corresponding entities from the database table. That means for each table in the database, there is a corresponding web page which communicates with the database (Figure 5-1). The following page allows the user to create a new file or edit an existing file of the data base table `Member Load Configuration’. 


\section{RCMemberLoadConfiguration}

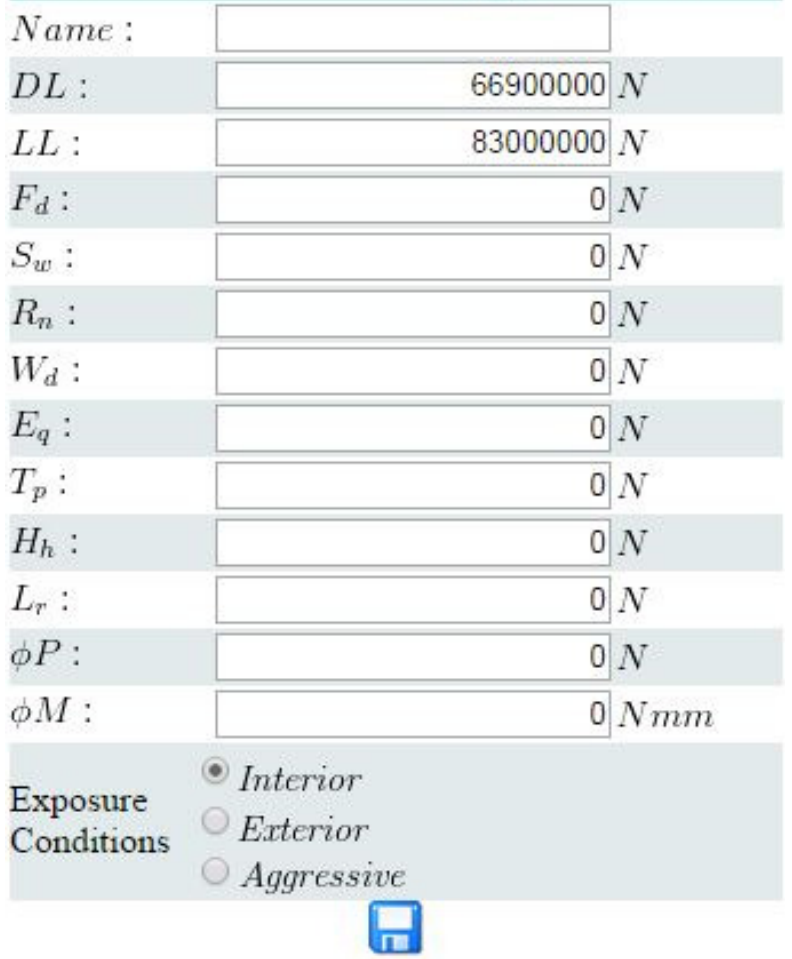

Figure 5-1: Page corresponding to table 'CADEC_RCMemberLoads'

For each type of collection there is a page which shows all the records in the database created by user. Figure 5-2 shows the webpage that views all the records stored by the user in the database table 'CADEC_RCMemberLoads' using the webpage as shown in Figure 5-1. 


\section{Member Loads}

\begin{tabular}{|c|c|c|c|c|c|c|c|c|c|c|c|c|c|}
\hline Name & $N$ & $N$ & $N$ & $N$ & $N$ & $N$ & $N$ & $N$ & $N$ & $N$ & $N$ & $N$ & $N$ \\
\hline ( & 100000000 & 176000000 & 0 & 0 & 0 & 0 & 0 & 0 & 0 & 0 & 0 & 0 & 0 \\
\hline (17) ExampleF-BeamShear & 24400 & 381000 & 0 & 0 & 0 & 0 & 0 & 0 & 0 & 0 & 0 & 0 & 0 \\
\hline (11) ExampleG-BeamShear & 92000 & 130000 & 0 & 0 & 0 & 0 & 0 & 0 & 0 & 0 & 0 & 0 & 0 \\
\hline (111) ExampleE-BeamShear & 72500 & 56700 & 0 & 0 & 0 & 0 & 0 & 0 & 0 & 0 & 0 & 0 & 0 \\
\hline (iii) ExampleB-BeamFlexure & 378000000 & 496000000 & 0 & 0 & 0 & 0 & 0 & 0 & 0 & 0 & 0 & 0 & 1 \\
\hline (iii) ExampleI-Column & 1420000 & 2560000 & 0 & 0 & 0 & 0 & 0 & 0 & 0 & 0 & 0 & 0 & 1 \\
\hline (111) ExampleL-BeamColumn & 0 & 0 & 0 & 0 & 0 & 0 & 0 & 0 & 0 & 0 & 520000 & 45000000 & 0 \\
\hline (i1) ExampleD-BeamFlexure & 90100000 & 205000000 & 0 & 0 & 0 & 0 & 0 & 0 & 0 & 0 & 0 & 0 & 0 \\
\hline (11) ExampleK-BeamColumn & 0 & 0 & 0 & 0 & 0 & 0 & 0 & 0 & 0 & 0 & 5200000 & 450000000 & 1 \\
\hline (1ii) ExampleH-Column & 950000 & 1280000 & 0 & 0 & 0 & 0 & 0 & 0 & 0 & 0 & 0 & 0 & 1 \\
\hline (iil ExampleJ-Column & 567000 & 2780000 & 0 & 0 & 0 & 0 & 0 & 0 & 0 & 0 & 0 & 0 & 1 \\
\hline (i1) ExampleA-BeamFlexure & 66980000 & 83350000 & 0 & 0 & 0 & 0 & 0 & 0 & 0 & 0 & 0 & 0 & 0 \\
\hline
\end{tabular}

Figure 5-2: View of all the records from 'CADEC_RCMemberLoads' in the web page

\subsection{Chapters}

While the styles of the webpages in this section are inherited from CADEC 14, the design procedures involve many more steps and many more properties when compared to the CADEC 14 layout. Hence, a new presentation model is introduced that is more intuitive, better organized with an end goal of making the job of the user easy, in spite of the complex and tedious nature of the methods that are involved. The design of FRP strengthening in all the cases of Beam Flexure, Beam Shear, Column Axial and Beam Column loading is carried out via a three step procedure. The following sections explain how the design procedures have been presented in the web page and discuss the validation and theory behind each step.

\subsubsection{Beam Flexure}

The first step of FRP strengthening of Beam Flexure design involves the input of moment loads acting on the member. A maximum of 13 types of loads could be taken as input [Section 2.2 FRP Load and Strength Design]. The loading configuration of a member can be stored in the 'My Documents' section, the user can retrieve all of the stored load configurations and apply the relevant configuration. The dropdown menu (RCLoad) from Figure 5-3 gives the list of load configurations from the database that can be identified by a given name. The user can also change the value of any load on this page but such changes will not be reflected in the database. The changed values will only effect further calculations. 


\begin{tabular}{|c|c|c|}
\hline \multirow{2}{*}{$\begin{array}{r}\text { Step1-Load Step } \\
\text { RCMemberLoads }\end{array}$} & \multirow[t]{2}{*}{ Step2-Properties Step } & Step3-Results Step \\
\hline & & \\
\hline RCLoad: & ExampleD-BeamFlexure & \\
\hline$D L:$ & 9010000 & $\mathrm{Nmm}$ \\
\hline$L L:$ & 20500000 & $\mathrm{Nmm}$ \\
\hline$F_{d}:$ & & $\mathrm{Nmm}$ \\
\hline$S_{w}:$ & & $\mathrm{Nmm}$ \\
\hline$R_{n}:$ & & $\mathrm{Nmm}$ \\
\hline$W_{d}:$ & & $\mathrm{Nmm}$ \\
\hline$E_{q}:$ & & $\mathrm{Nmm}$ \\
\hline$T_{p}:$ & & $\mathrm{Nmm}$ \\
\hline$H_{h}:$ & & $\mathrm{Nmm}$ \\
\hline$L_{r}:$ & & $\mathrm{Nmm}$ \\
\hline
\end{tabular}

Next

Figure 5-3: Step 1 of Beam Flexure Design

The second step allows the user geometric properties, steel reinforcement properties of concrete and properties of FRP. User can select a concrete member configuration and FRP properties from corresponding dropdowns (RCMember, FRP Properties) as shown in Figure 5-4, which are then retrieved from the data base.

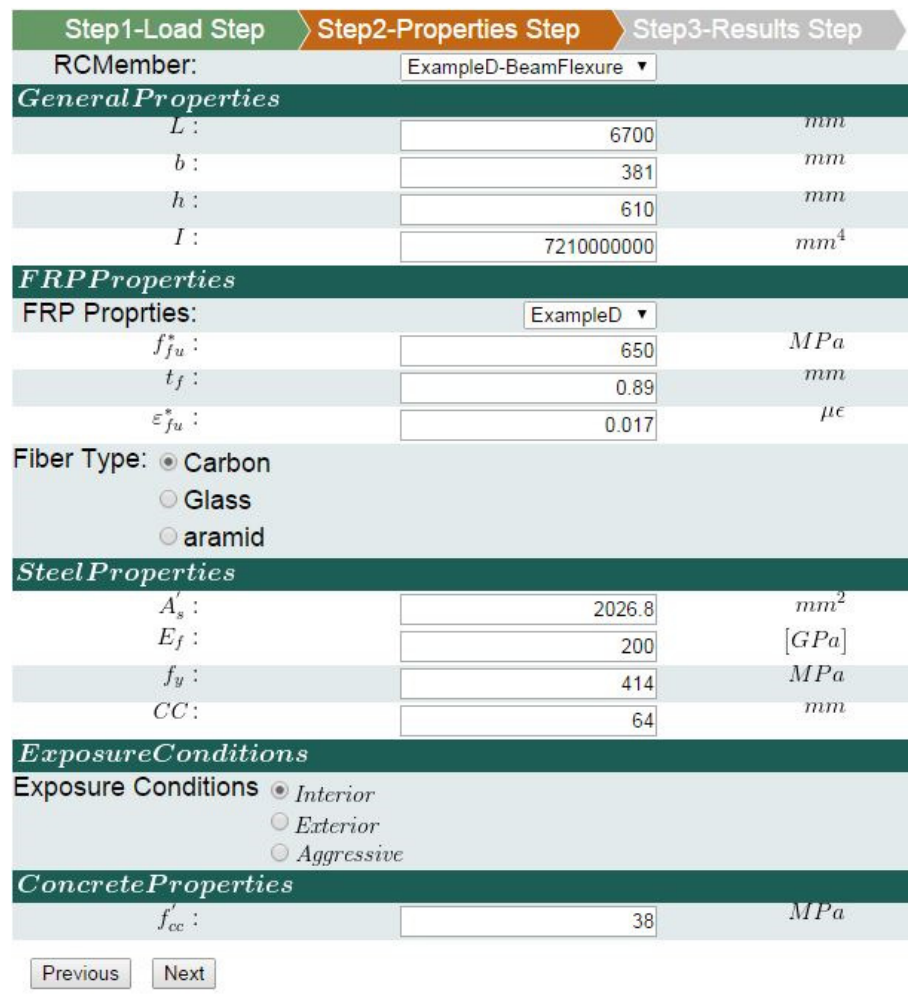

Figure 5-4: $2^{\text {nd }}$ Step of Beam Flexure Strengthening- Defining properties of the member. 
At the end of the second step, initial checks for the design are performed. The program triggers the calculations of factored load capacity and factored moment capacity [Section 2.4 Flexure Strengthening] and checks for reliability condition as in ACI rules [Section 2.2 FRP Load and Strength Design]. The program also calculates strengthening limits [Section 2.4 Flexure Strengthening] and checks for maximum loading condition. It further calculates the minimum number of layers required in Balanced Strengthening Configuration [2.4 Flexure Strengthening].

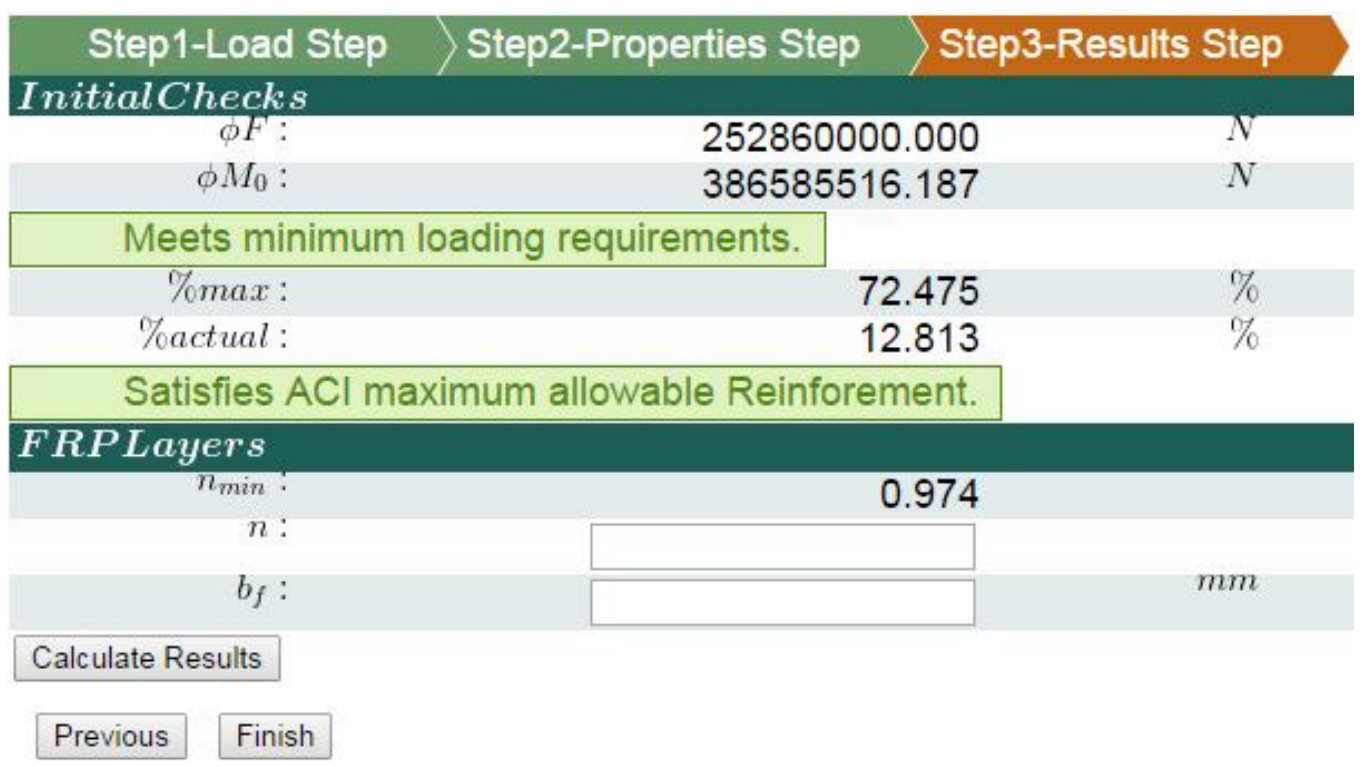

Figure 5-5: $3^{\text {rd }}$ Step of Beam Flexure Strengthening-Initial Checks.

The Serviceability conditions are checked after the user has entered the required number of FRP layers and the width of the ply. While all the entities from the Loads Step and the Properties Step are saved, none of the entities from the Result step are saved in the database. The function of the Result step is to check the design for serviceability conditions with the user defined number of FRP layers. Figure 5-6 shows the complete set of checks performed by the program after user input. 


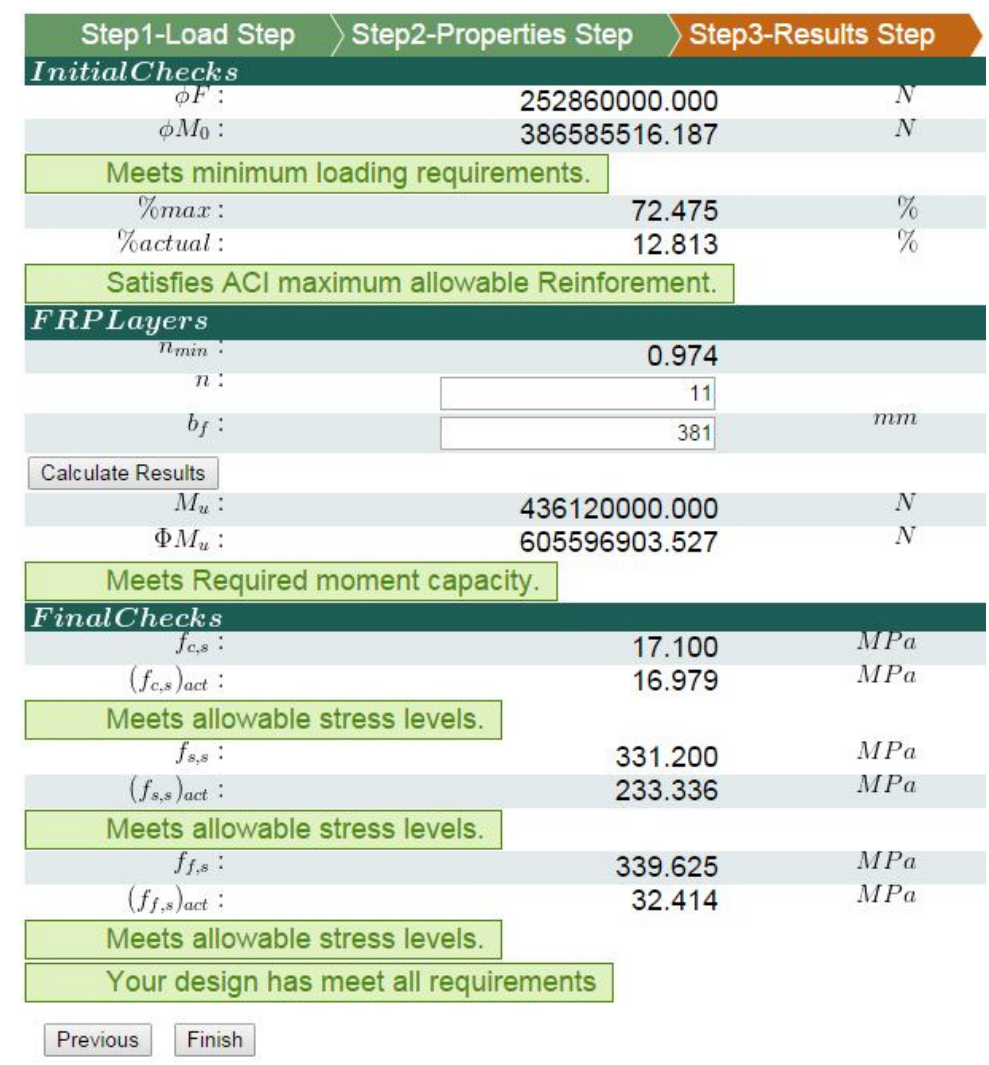

Figure 5-6: $3^{\text {rd }}$ Step of Beam Flexure Strengthening- Serviceability Checks

The user can try and change the number of layers required and the width of the ply to check how the results vary and eventually come up with an optimized model.

\subsubsection{Beam Shear Web Page}

Once a beam is strengthened for its flexure strength, it is then designed for shear strengthening if necessary or vice versa. In the 3 step design process, load configuration is selected from the dropdown menu (RCLoad) as shown in Figure 5-7. For a beam in shear, the loads are in terms of force (Newtons). 


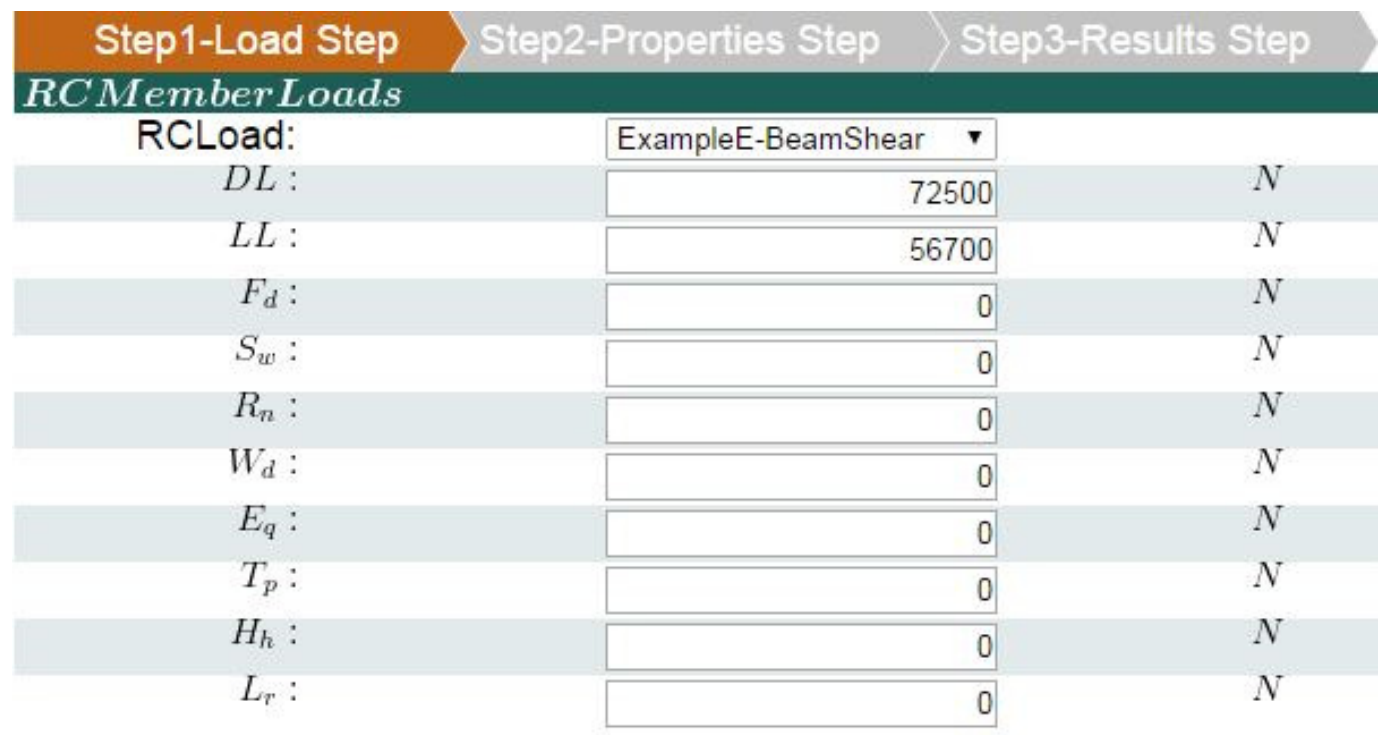

\section{Next}

Figure 5-7: $1^{\text {st }}$ step in Beam Shear Strengthening- Defining load configuration

In the second step, the geometric, steel reinforcement, concrete and FRP properties are defined for the model (Figure 5-8). The geometric properties are required to calculate neutral axis [Section 2.4 Flexure Strengthening]. The Type of FRP and the exposure to the environment determines the reduction of strength due to environmental conditions [Section 2.3 Environmental Reduction Factor, Table 2.2- Environmental Reduction Factor . The steel properties are required for the calculation of the strengthening provided by the steel reinforcement. FRP strengthening finds its use only after the cracks appear i.e. post cracking most of the stresses are taken by the FRP. 


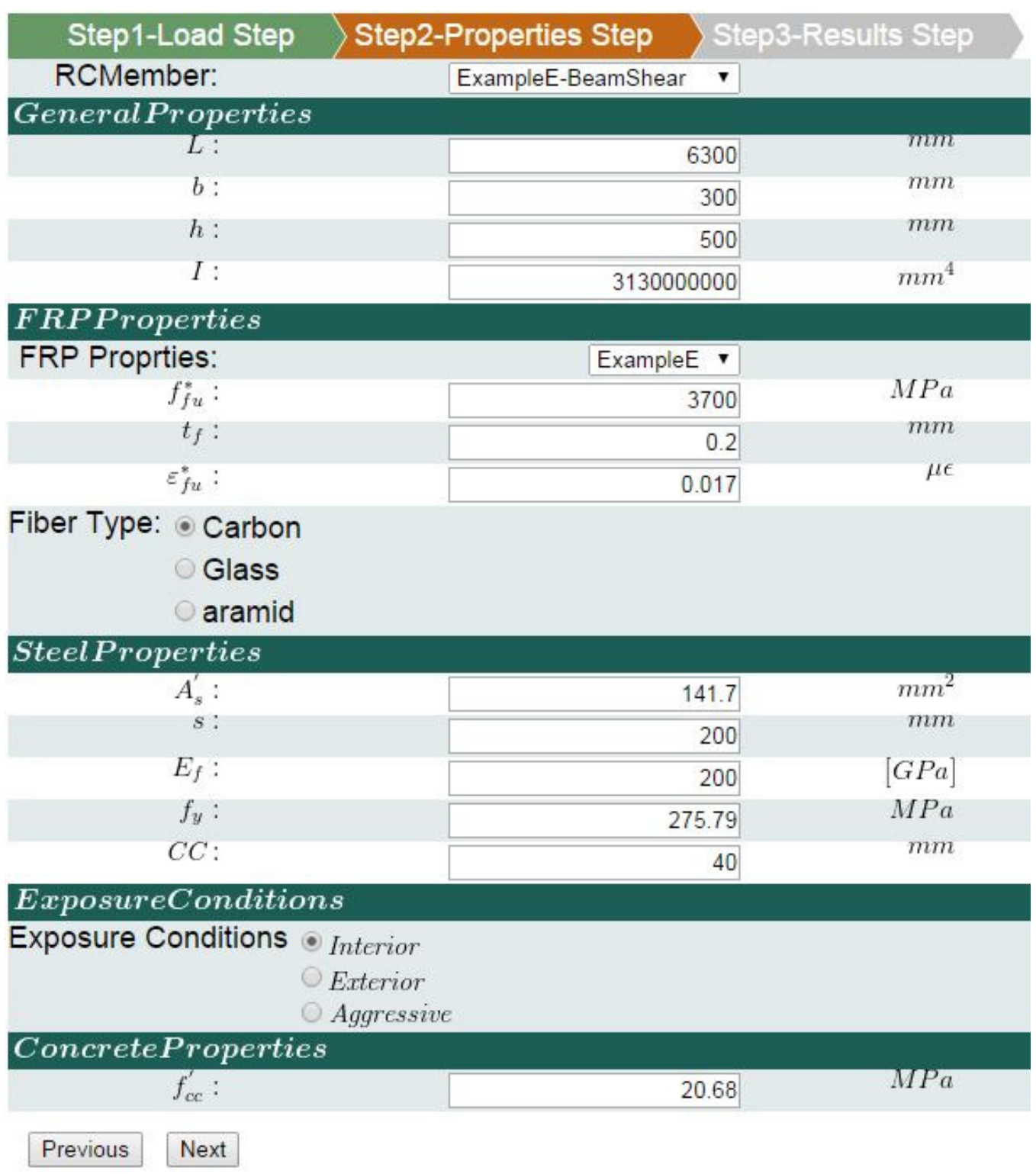

Figure 5-8: $2^{\text {nd }}$ Step in Beam Shear Strengthening - Defining properties of the member

At the end of second step, initial checks are carried out by the program to check whether the design stands good for the minimum requirements of ACI, viz. the design checks for maximum allowable shear strengthening, a limit set by ACI and the factored loading to satisfy LFRD condition [Section 2.2 FRP Load and Strength Design]. For discontinuous wrapping, the user is allowed to change the angle of wrapping, and the spacing between the subsequent wrappings. For any wrapping style other than complete wrapping, the user is allowed to change the width of the wrapping and customize the model to achieve the optimal design. In addition to the basic checks for the design, the program calculates the minimum layers required for the model, based on the 'Style of Wrapping' and 'Wrapping Continuity' (Figure 5-9) options chosen in the member configuration (Step 2). The design is then checked for serviceability. 


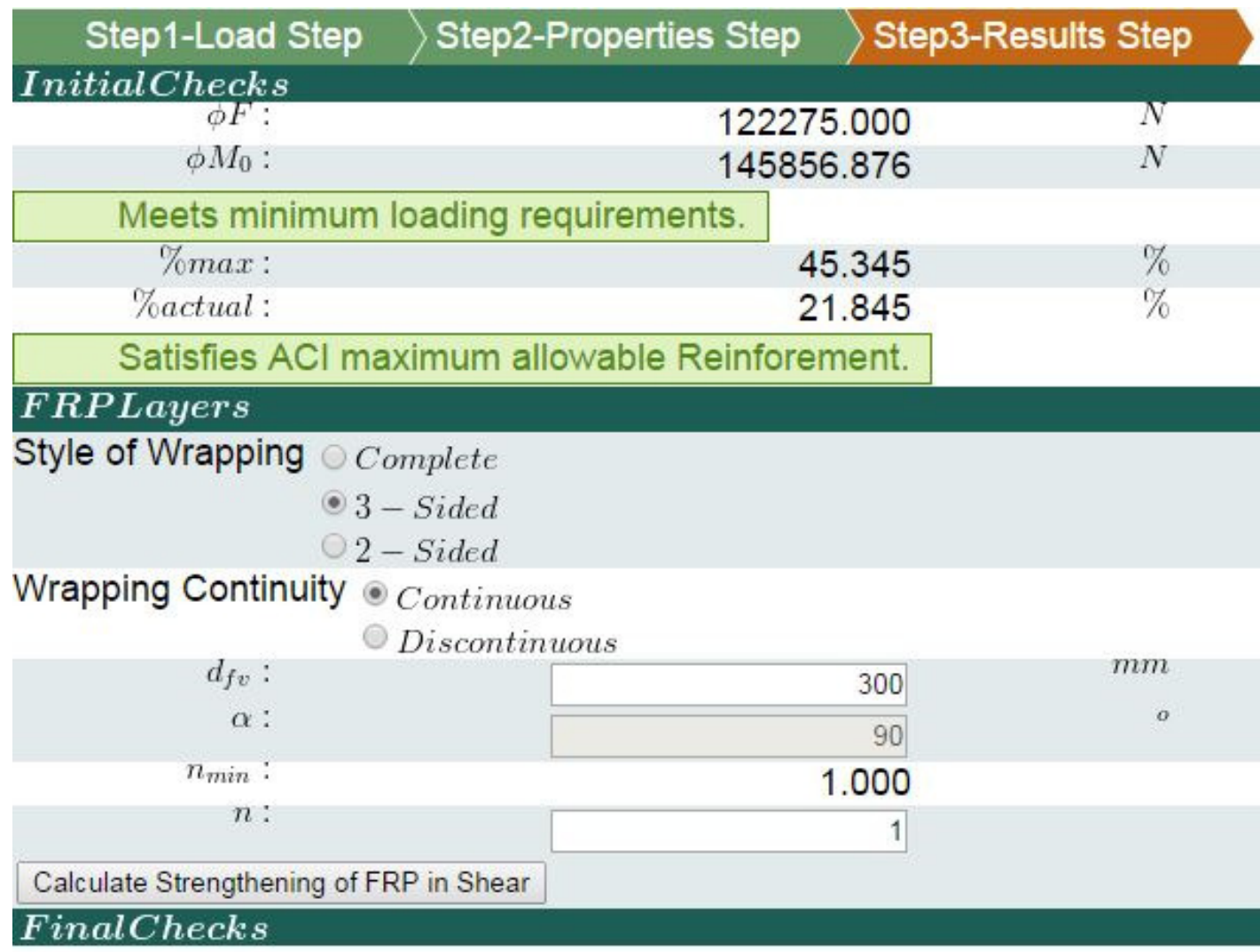

Previous Finish

Figure 5-9: $3^{\text {rd }}$ step in Beam Shear Strengthening - Initial checks

Once the variables are set, on the click of the 'Calculate Strengthening of FRP in Shear' button, the final checks for the design are performed [Section 2.5 Shear Strengthening]. The shear design checks for the maximum FRP shear reinforcement provided, against the FRP shear reinforcement required. Also, for the ACI limit check, the reinforcement strength provided by the steel and FRP must not cross the limit prescribed by ACI [Section 2.5 Shear Strengthening]. If the limits and checks are satisfied, then the design abides by all the ACI requirements and design requirements. The step by step design procedure for shear design is presented in Figure 5-7, Figure 5-8, Figure 5-9, Figure 5-10. 


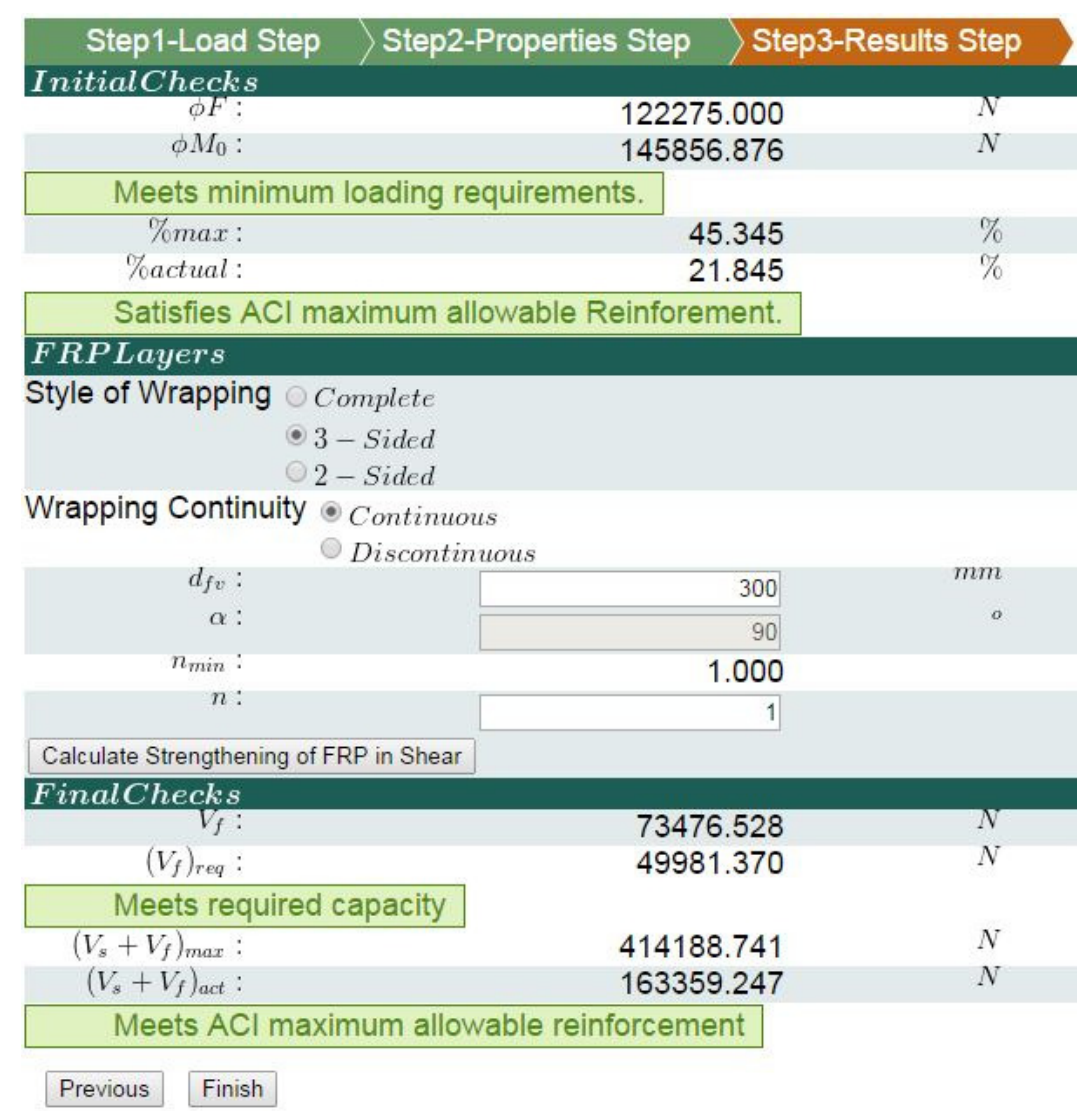

Figure 5-10: $3^{\text {rd }}$ Step in Beam Shear Design and Analysis- Serviceability Checks

\subsubsection{Column Axial Design- Web Page}

In this design, a column is strengthened for only for axial stresses. Loads that are considered in the design process are similar to those for beam. This design considers all the dead and dynamic loads. In the first step, the load configuration is selected for the model (Figure 5-11).

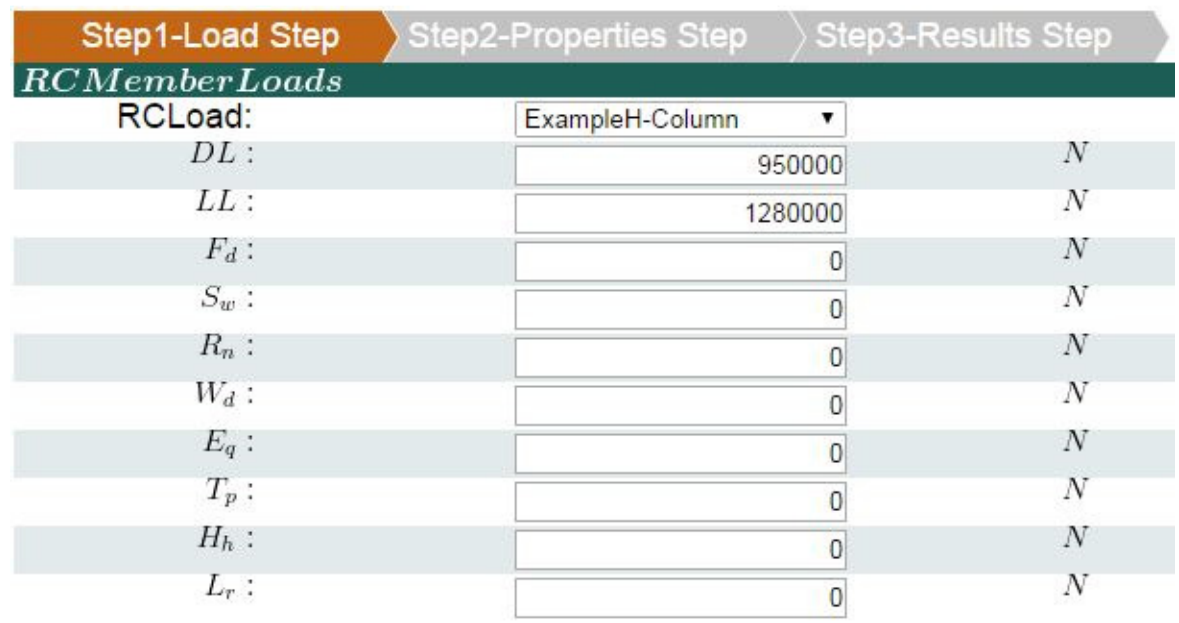

Next

Figure 5-11: $1^{\text {st }}$ step in Column Axial Strengthening- Defining Loading Configuration 
In the second step, the geometric, concrete, steel reinforcement and FRP reinforcement properties for the model are defined by choosing a member configuration and FRP configuration from the dropdown menu (Figure 5-12: $2^{\text {nd }}$ step of Column Axial Load Design - Defining properties of the member.Figure 5-12). The geometric properties required here are different from the ones required for beam strengthening.

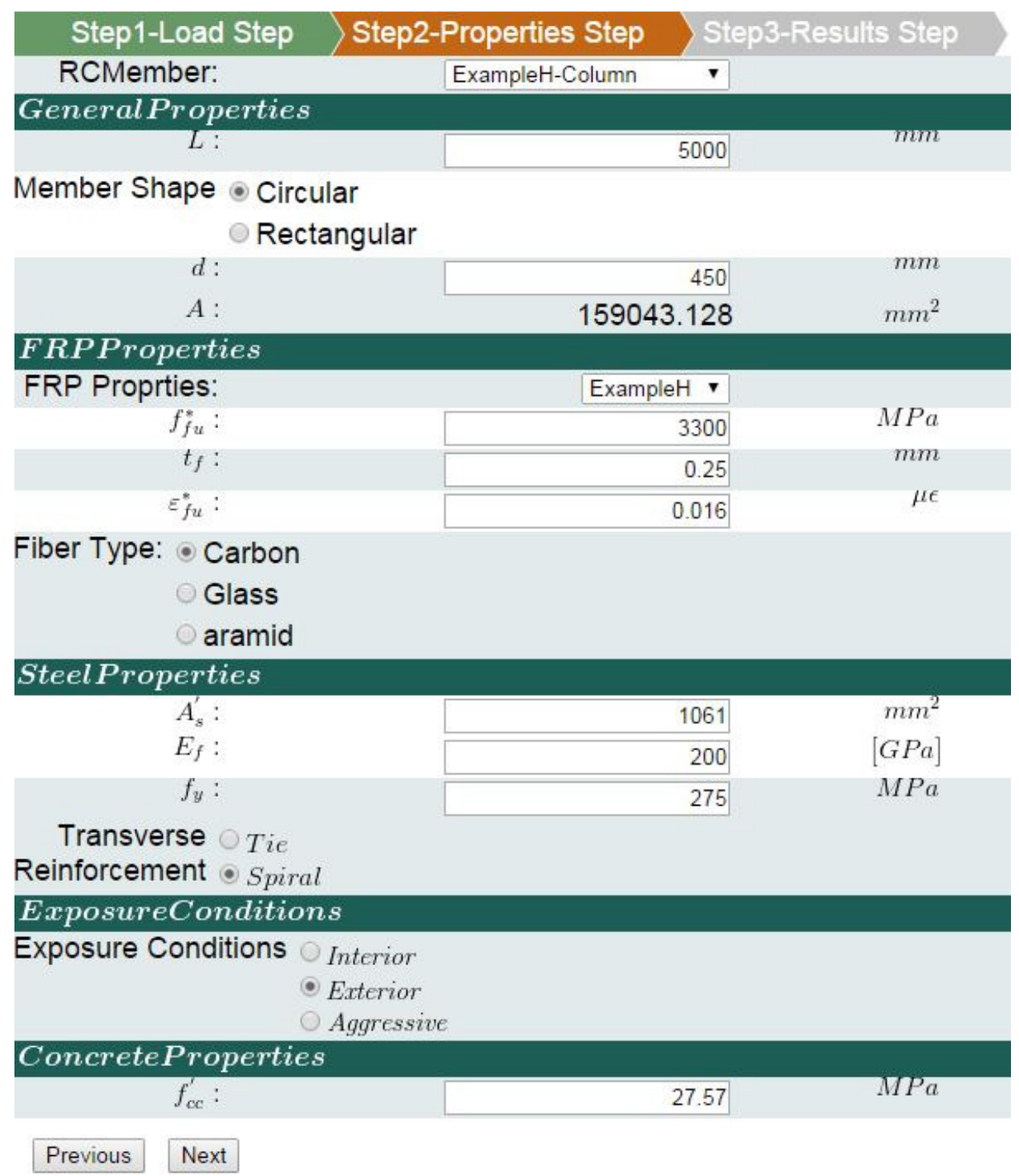

Figure 5-12: $2^{\text {nd }}$ step of Column Axial Load Design - Defining properties of the member.

At the end of step 2, initial checks are performed (Figure 5-13). The factored initial capacity must be greater than the factored load. The maximum strengthening must be greater than the actual strengthening. Also the program calculates the minimum number of layers required, while the user can customize the required number of layers for the system. Later, the design is checked for serviceability conditions. 


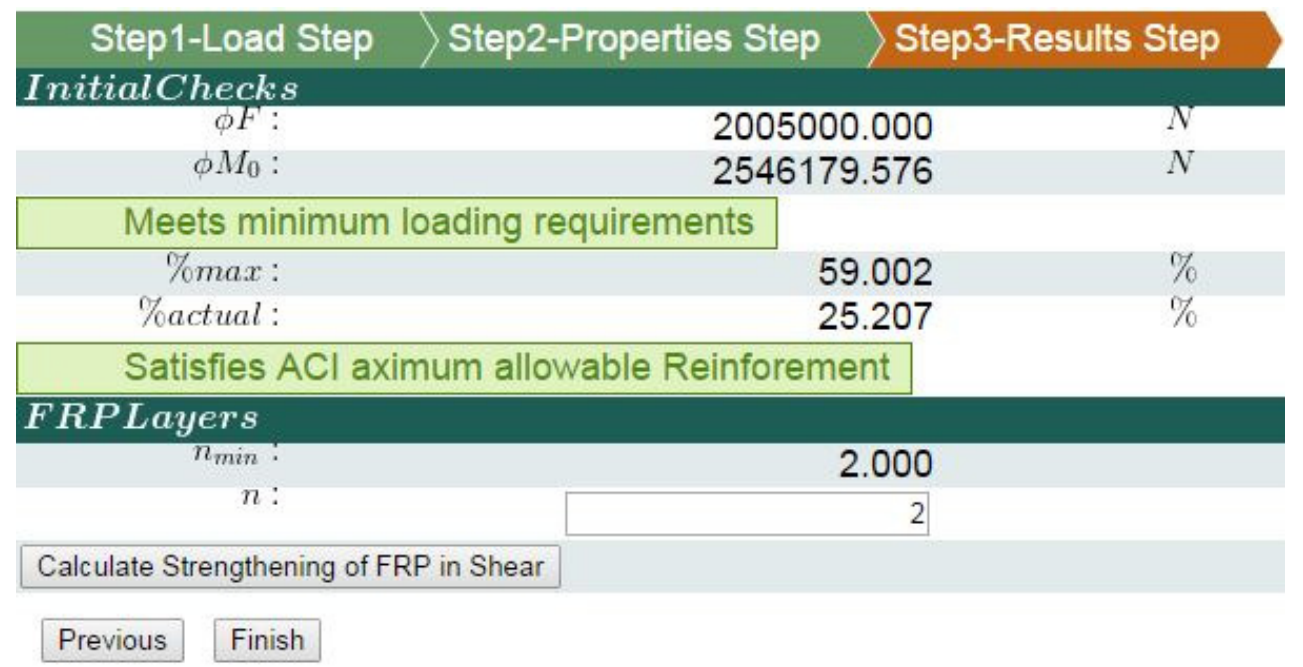

Figure 5-13: $3^{\text {rd }}$ Step in Column Axial Strengthening Design- Initial Checks

The serviceability conditions (Figure 5-14) for the column under axial stress include the conditions that satisfy the load capacity check [Section Error! Reference source not found.], the maximum compressive strain check and the confinement ratio check. The maximum compressive strain in concrete and steel is limited to prevent the premature cracking in the FRP. ACI limits the FRP strength in shear which is the function of confinement ratio [Section Error! Reference source not found.]. 


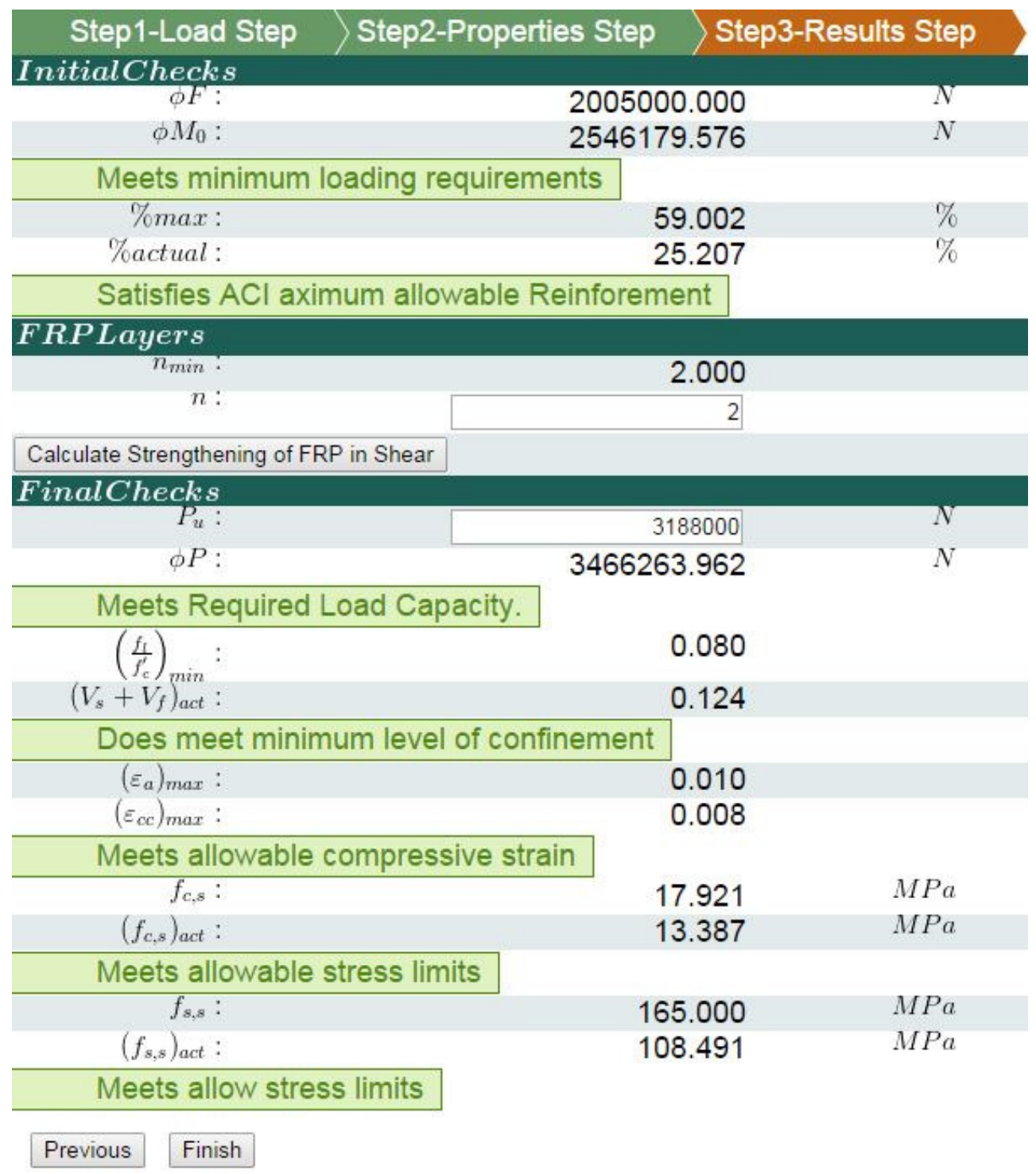

Figure 5-14: $3^{\text {rd }}$ step in Column Axial Strengthening- Serviceability checks

\subsubsection{Beam-Column Axial Design- Web Page}

In this design, a column is strengthened for only for bending and axial stresses. This design considers factored axial load and factored moment load. In the first step, the load configuration is selected for the model (Figure 5-11).

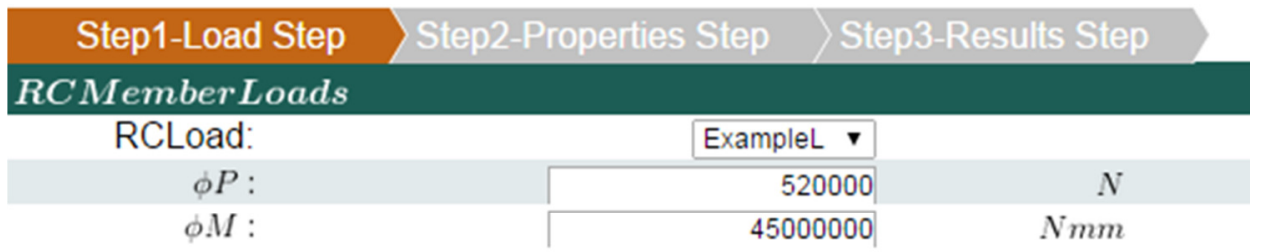

Next

Figure 5-15: 1st step in Beam-Column Strengthening- Defining Loading Configuration 
Beam-Column strengthening is verification procedure. In this method, procedure does not suggest required number of FRP layers. This procedure verifies if specified number of FRP layers sustains the design or not.

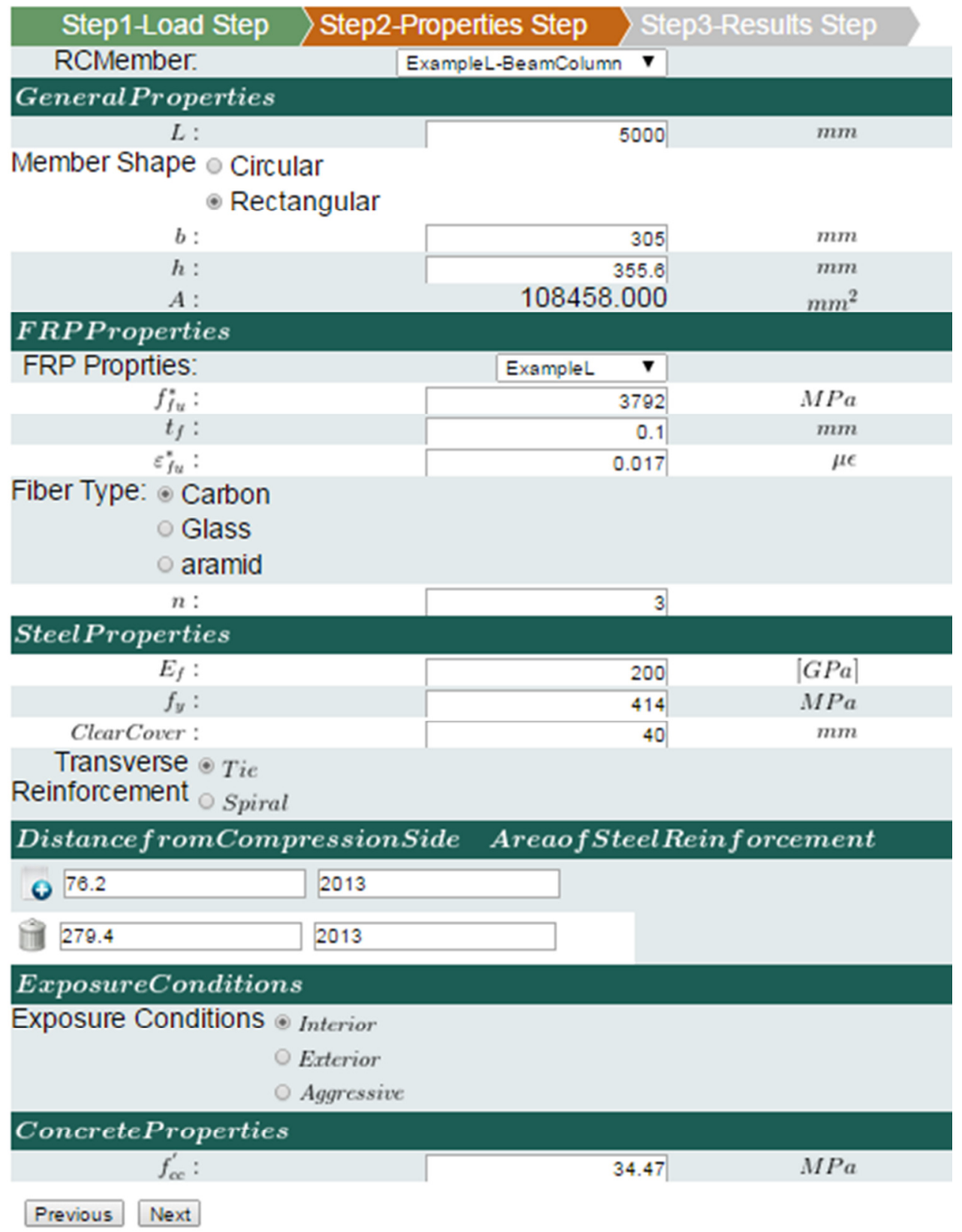

Figure 5-16: 2nd step in Beam-Column Strengthening- Defining Member Configuration

The results page is basically a plot showing where the design stands from the standards. (Figure 5-117) shows the example of verification procedure. 


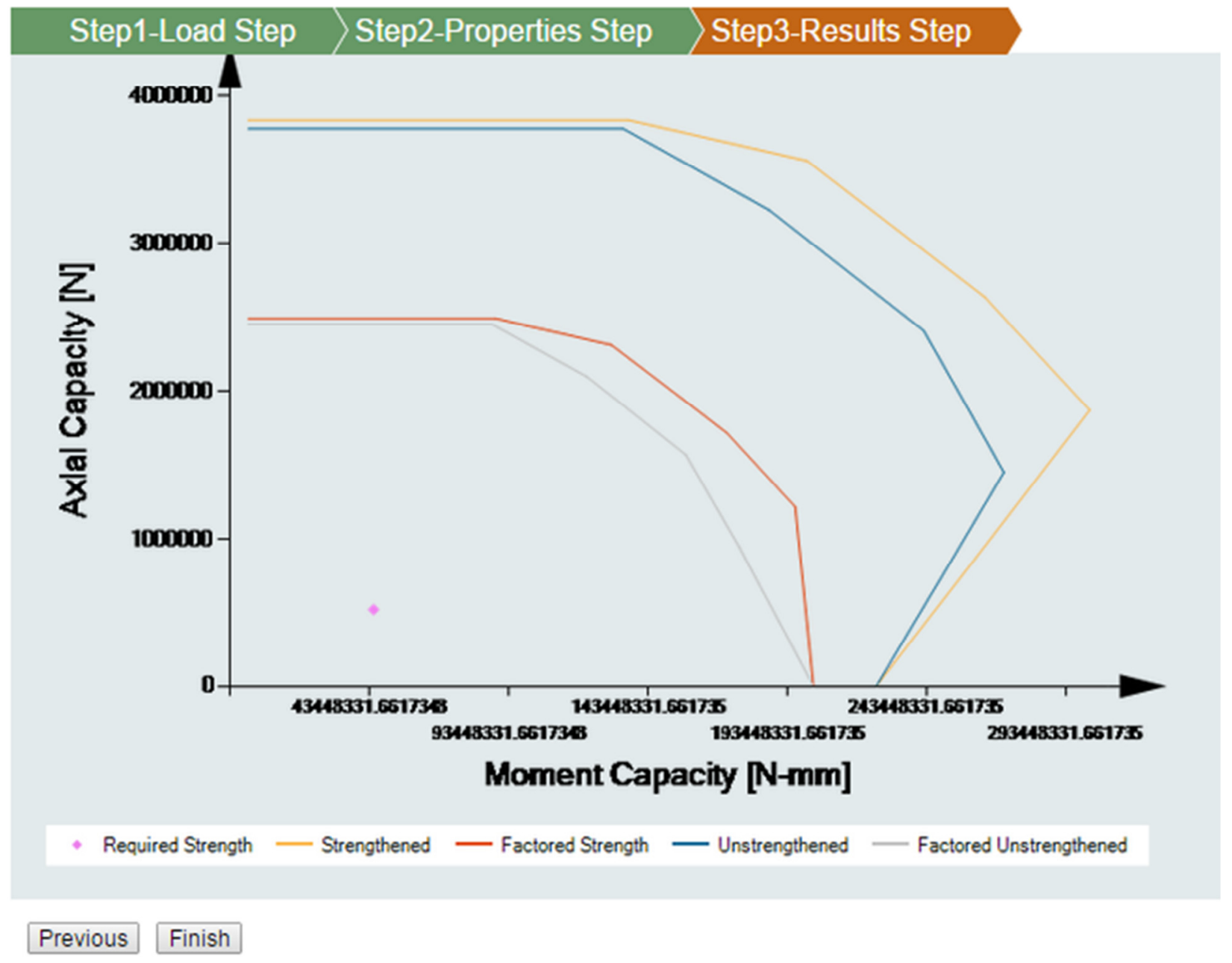

Figure 5-17: 3rd step in Beam-Column Strengthening- Verification 


\section{Chapter 6 : Verification}

Examples from (Saas, 2013) are used to verify the web application. Following figures illustrate the use of the WebApp to analyze several examples taken from (Saas, 2013). Each example concludes with results that coincide with those obtained by (Saas, 2013). The final results are listed at the end of each example to highlight the conclusion of each example.

\subsection{Examples for Beam in Flexure:}

Beam Example 1:

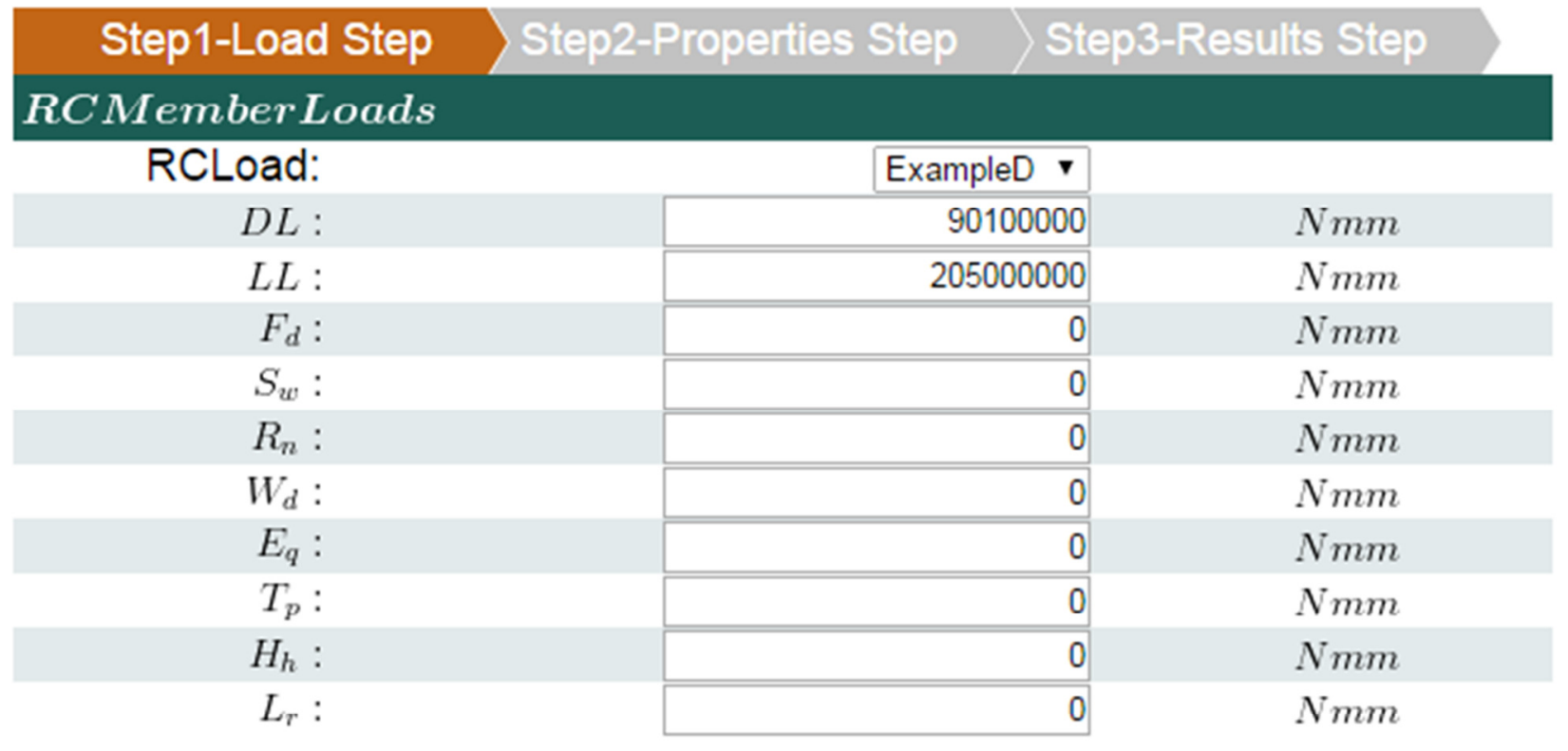

Next

Figure 6-1: Beam Flexure- Step 1. 


\section{Step1-Load Step >Step2-Properties Step $>$ Step3-Results Step}

RCMember:

General Properties

$L:$
$b:$
$h:$
$I:$
$f_{f u}^{*}:$
$t_{f}:$
$f_{f u}^{*}:$

Fiber Type: Carbon

\section{FRPProperties}

FRP Proprties:

$f_{f u}^{*}$ :

$t_{f}:$

fu
ExampleD-Flexure $\mathbf{}$

\section{Glass \\ aramid}

\section{SteelProperties}

\begin{tabular}{r|r|r|}
\hline$A_{s}^{\prime}:$ & 2026.83 & $\mathrm{~mm}^{2}$ \\
$E_{f}:$ & 200 & {$[G P a]$} \\
\cline { 2 - 3 }$f_{y}:$ & 414 & $M P a$ \\
\hline ClearCover $:$ & 64 & $\mathrm{~mm}$ \\
\hline
\end{tabular}

ClearCover :

\begin{tabular}{|r|r|}
\hline ExampleD & \\
\hline 620.55 & $M P a$ \\
\hline 0.89 & $m m$ \\
\hline \hline 0.017 & $\mu \epsilon$
\end{tabular}

$m m$

$m m$

$m m$

$m m^{4}$

ExposureConditions

Exposure Conditions Interior

Exterior

Aggressive

ConcreteProperties

$f_{c c}^{\prime}$ :

38

$M P a$

Previous Next

Figure 6-2: Beam Flexure- Step 2. 


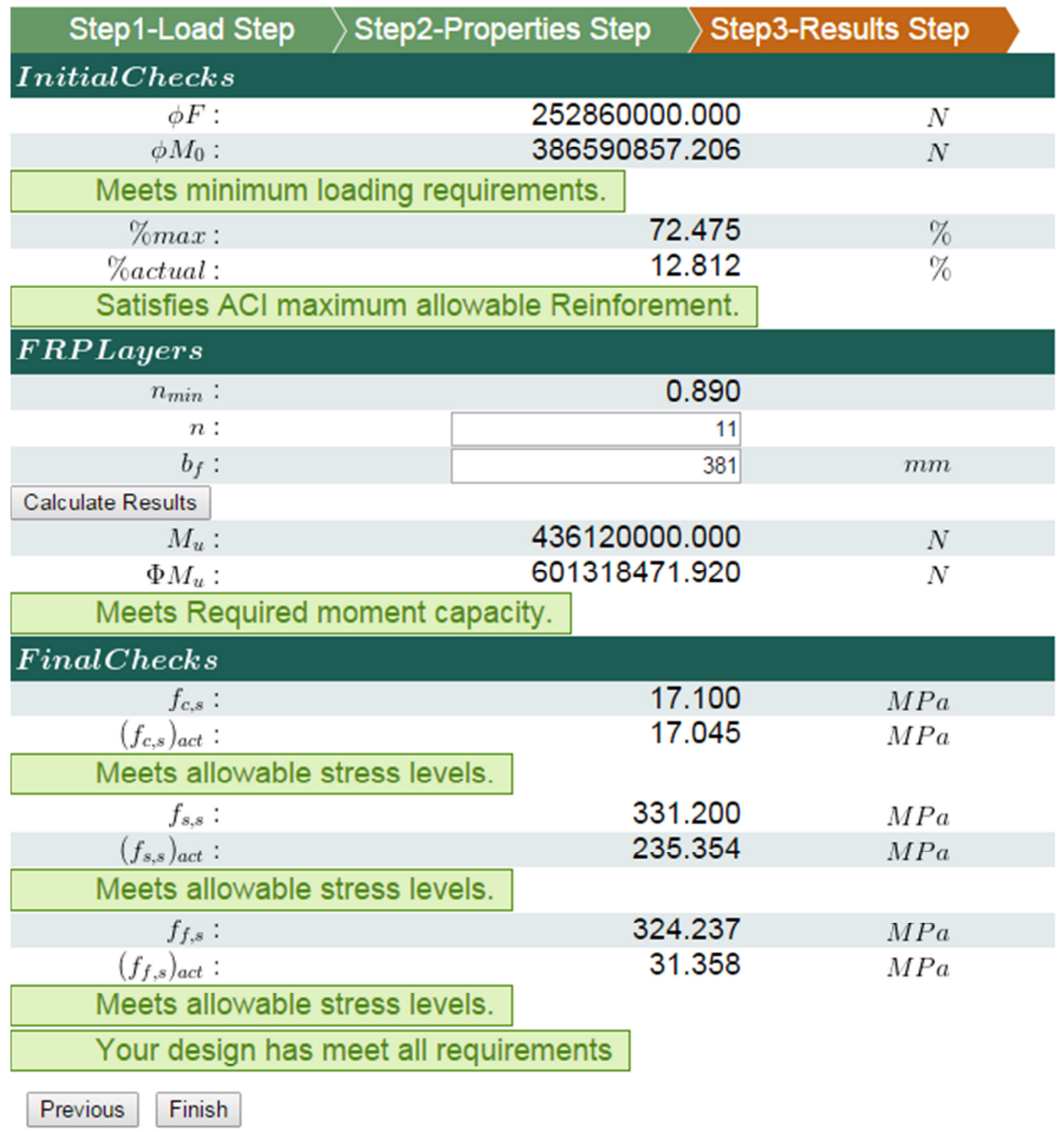

Figure 6-3: Beam Flexure- Step 3. 
Examples for Beam in Shear:

Example 1:

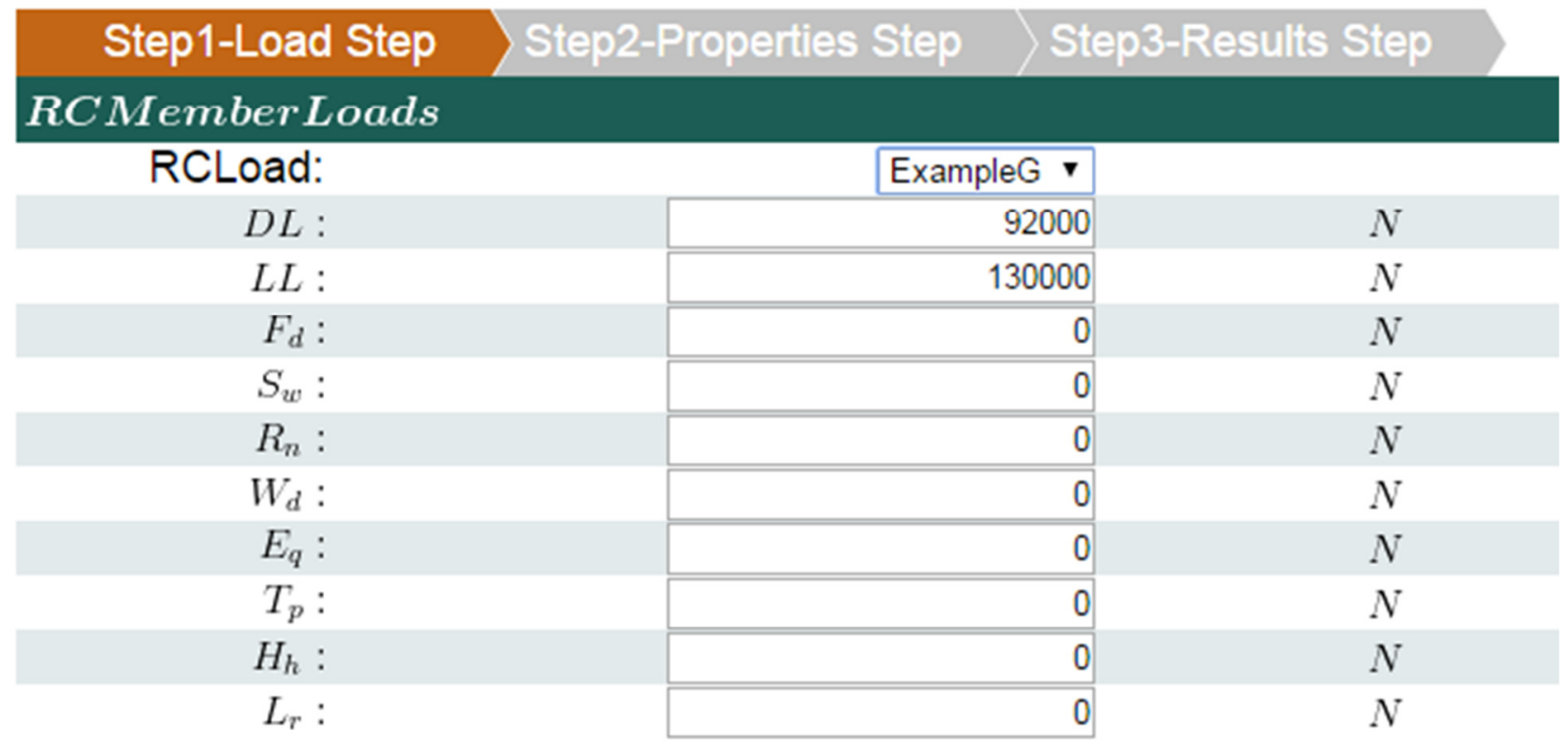

Next

Figure 6-4: Beam Shear- Step 1. 


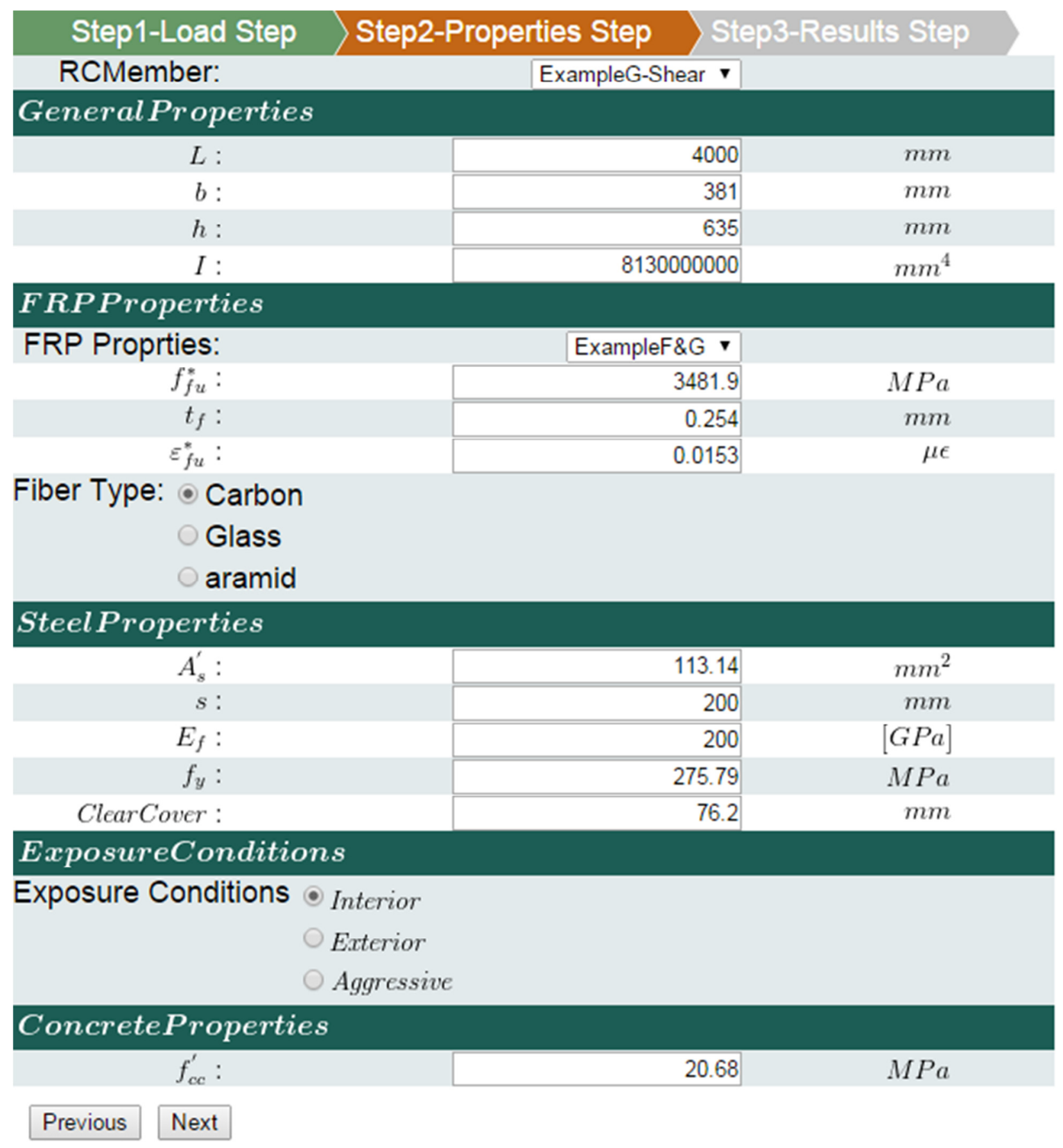

Figure 6-5: Beam Shear- Step 2. 


\begin{tabular}{|c|c|c|}
\hline Step1-Load Step & Step2-Properties Step & Step3-Results Step \\
\hline \multicolumn{3}{|l|}{ InitialChecks } \\
\hline$\phi F:$ & 19870 & 000 \\
\hline$\phi M_{0}:$ & 18640 & 285 \\
\hline \multicolumn{3}{|c|}{ Does not meet minimum loading requirements. } \\
\hline$\% \max :$ & & 242 \\
\hline \%actual: & & 808 \\
\hline Too much reinfor & ment needed. & \\
\hline
\end{tabular}

\section{FRPLayers}

Style of Wrapping Complete

$$
\begin{aligned}
& \text { 3-Sided } \\
& \text { - } 2-\text { Sided }
\end{aligned}
$$

Wrapping Continuity $\bigcirc$ Continuous

\begin{tabular}{|c|c|c|}
\hline$d_{f v}:$ & 406.4 & $m m$ \\
\hline$b_{f}:$ & 305 & $\mathrm{~mm}$ \\
\hline$\alpha:$ & 90 & o \\
\hline$s_{f}^{\max }:$ & 341.609 & $m m$ \\
\hline$s_{f}:$ & 500 & $m m$ \\
\hline$n_{\min }:$ & 3.000 & \\
\hline$n:$ & 3 & \\
\hline \multicolumn{3}{|c|}{ Calculate Strengthening of FRP in Shear } \\
\hline \multicolumn{3}{|l|}{ FinalChecks } \\
\hline$V_{f}:$ & 141457.248 & $N$ \\
\hline$\left(V_{f}\right)_{\mathrm{req}}:$ & 207045.828 & $N$ \\
\hline \multicolumn{3}{|c|}{ Does not meet required capacity } \\
\hline$\left(V_{s}+V_{f}\right)_{\max }:$ & 638999.585 & $N$ \\
\hline$\left(V_{s}+V_{f}\right)_{\text {act }}:$ & 228638.096 & $N$ \\
\hline \multicolumn{2}{|c|}{ Meets $\mathrm{ACl}$ maximum allowable reinforcement } & \\
\hline Previous Finish & & \\
\hline
\end{tabular}

- Discontinuous

Figure 6-6: Beam Shear- Step 3. 
Examples of Colum in Axial Loading:

Example 1:

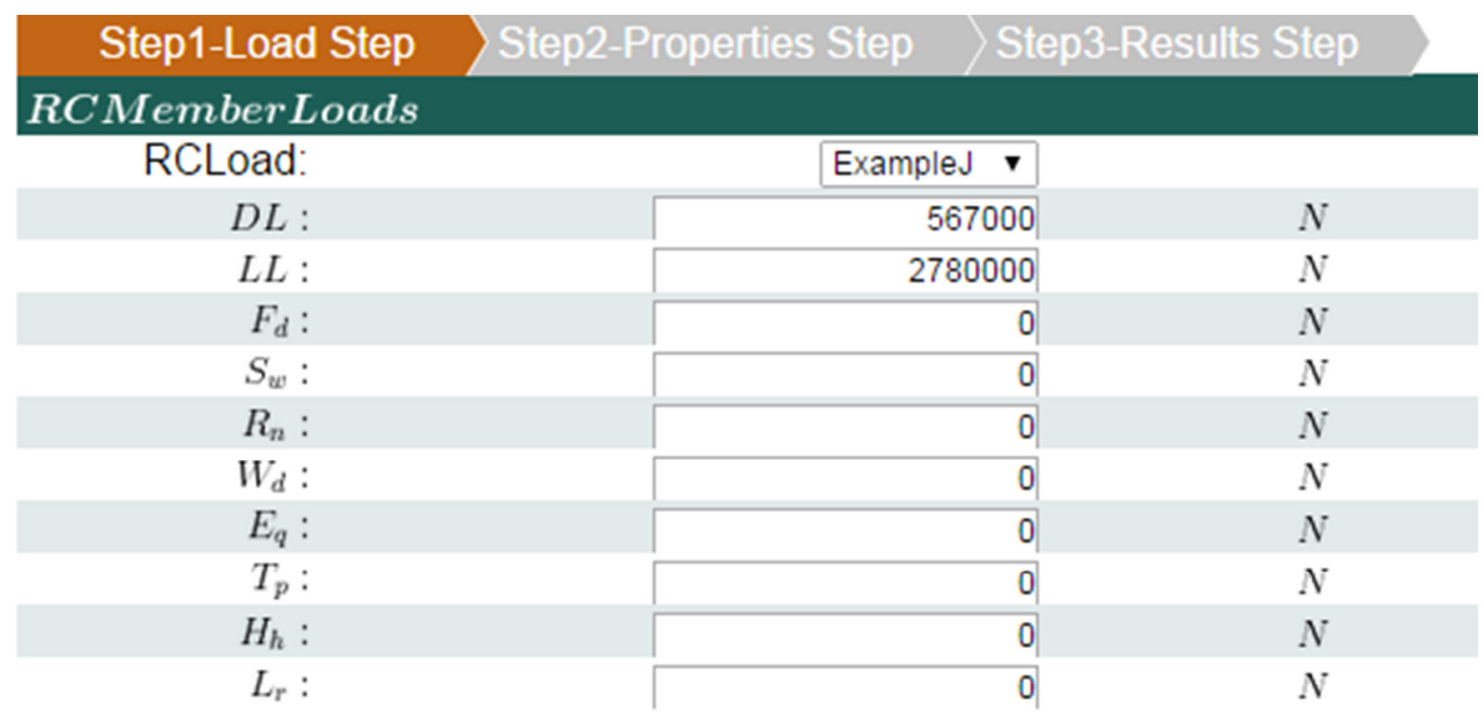

Next

Figure 6-7: Column- Step 1. 


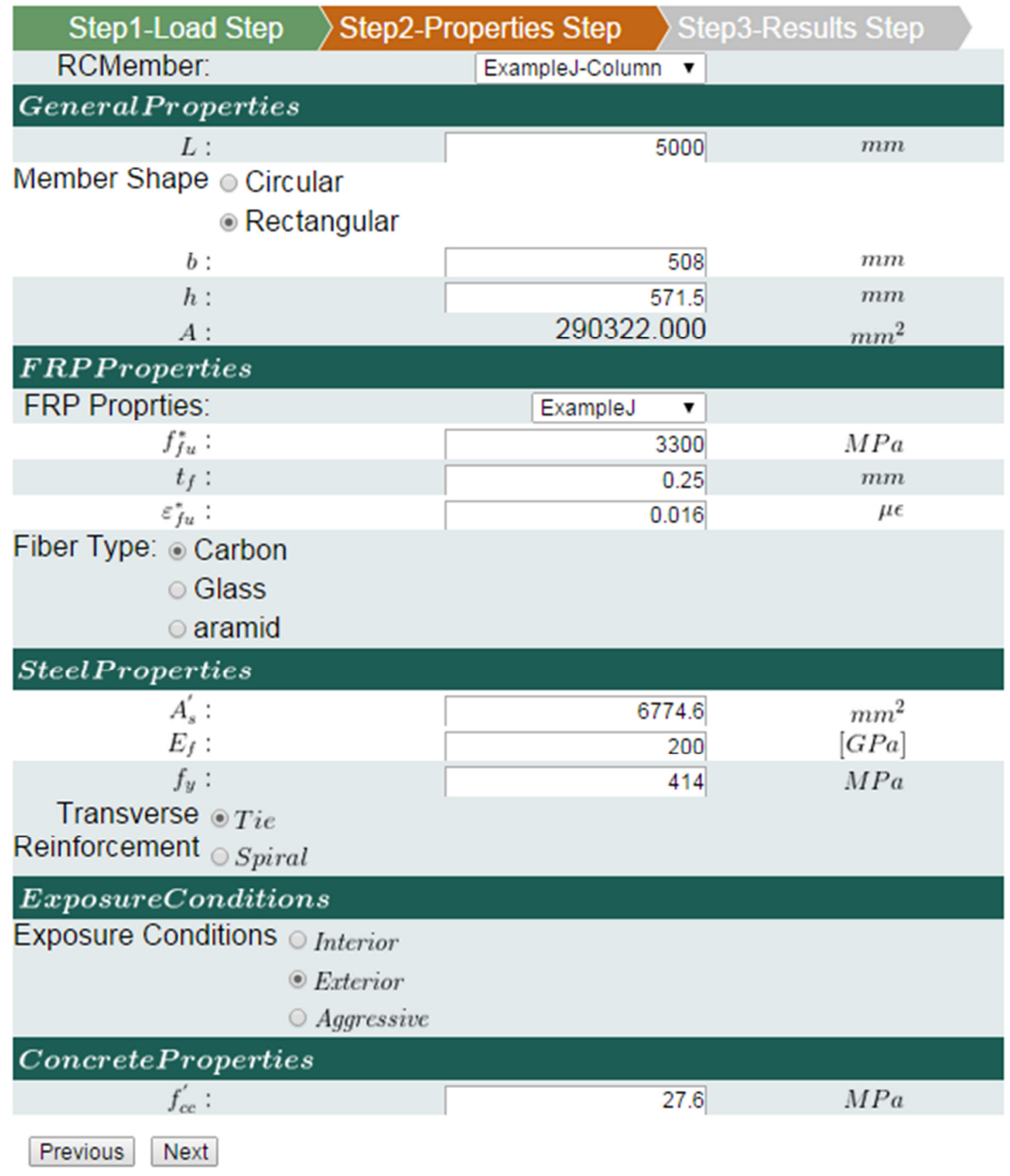

Figure 6-8: Column- Step 2. 


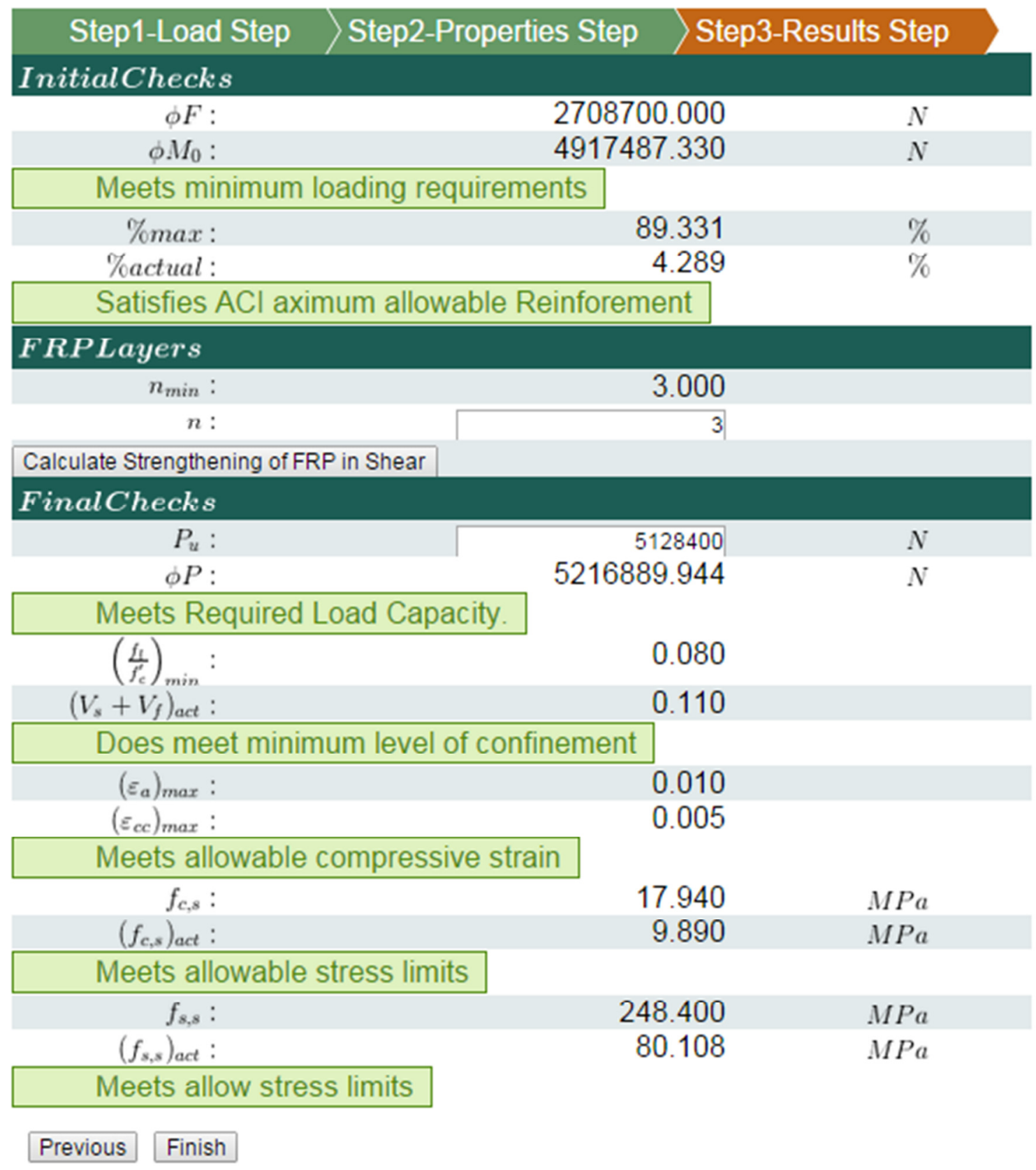

Figure 6-9: Colum- Step 3. 
Examples of Colum in Axial and Bending Loading:

Example 1:

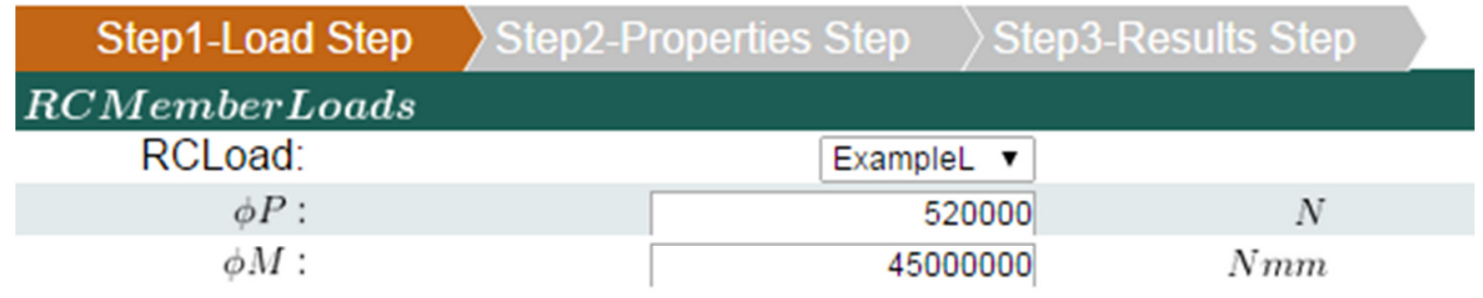

Next

Figure 6-10: Beam-Colum- Step 1.

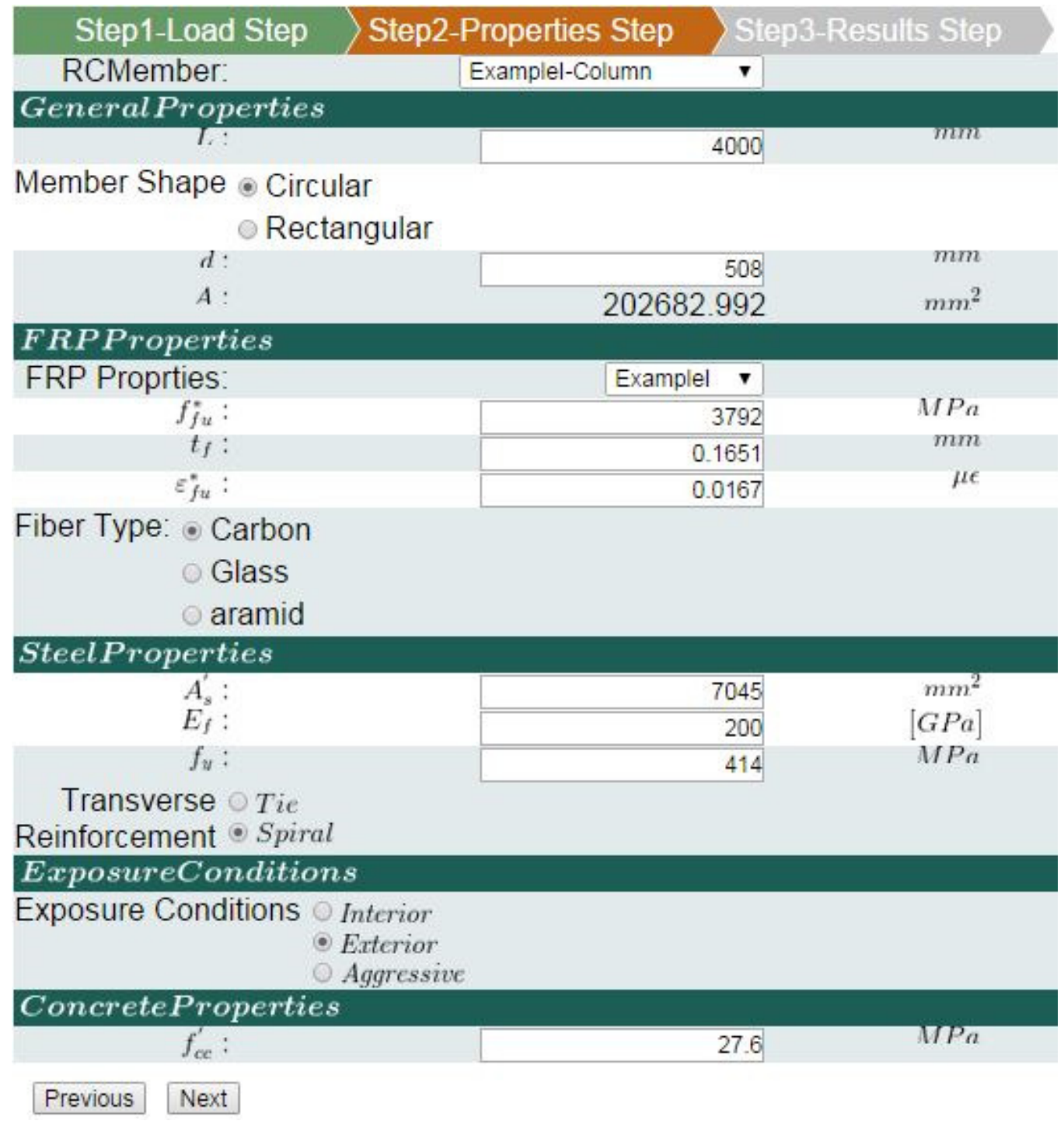

Figure 6-11: Beam-Colum- Step 2. 


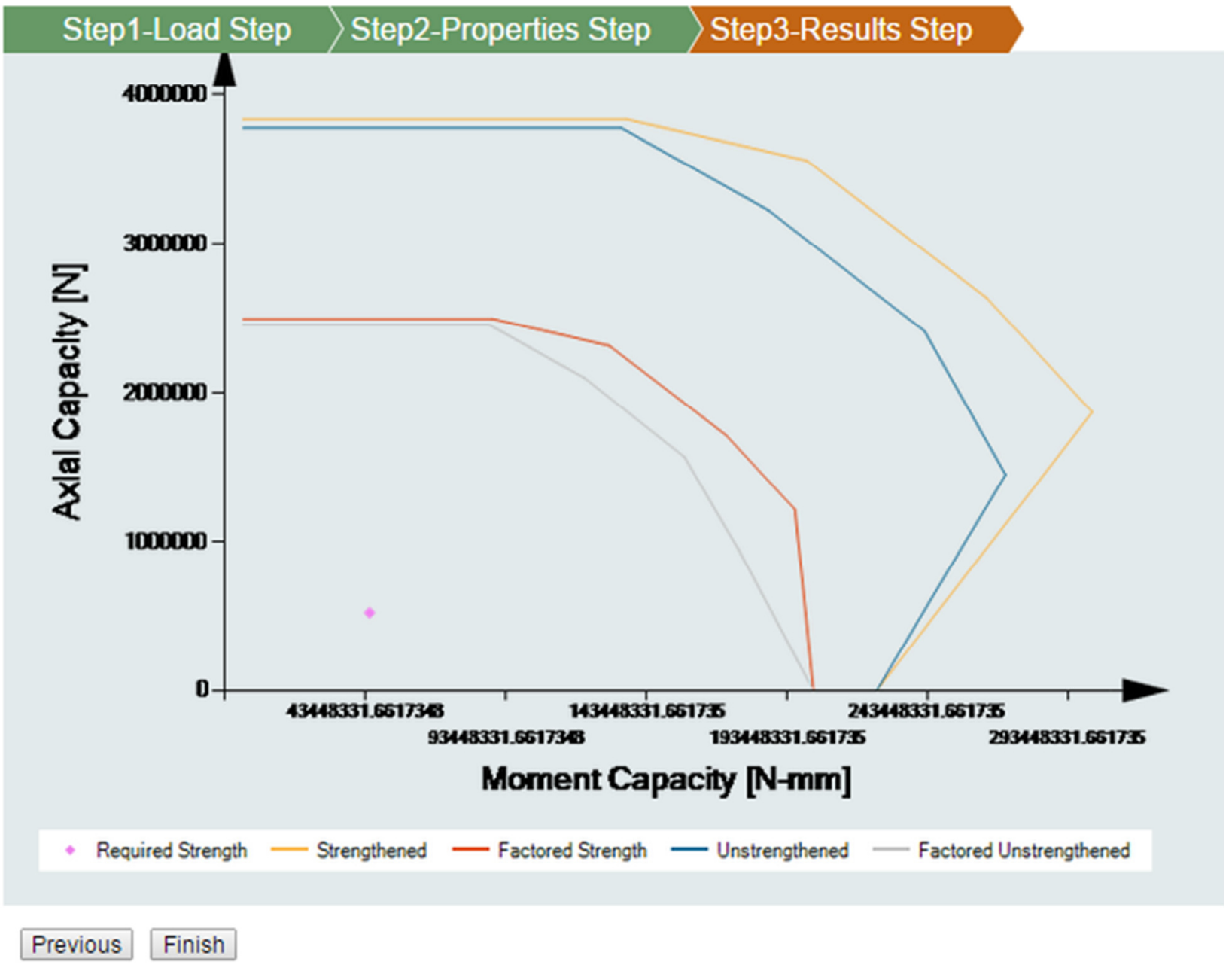

Figure 6-12: Beam-Colum- Step 3. 


\section{Chapter 7 : Conclusions and Future Work}

\subsection{Conclusions}

Through the present work, a web application is developed as a standalone 'add-on' module to the web application CADEC. The application facilitates the designing of FRP strengthening of a RC member with procedures described in the Chapter 'Strengthening of Reinforced Concrete' from book Introduction to Composite Materials. The software methods which implement the design procedures have been developed by (Saas, 2013). The application is built by inheriting the existing CADEC architecture.

The front-end architecture or the presentation layer for CADEC is redesigned to implement object oriented methods in the front end design. The new architecture is based on Server Control Concepts. While, the look of the webpages is retained, the code that generates the web page has been revised. The new serve control architecture makes the web page render much faster in the web browser than the previous version. Coding standards have been improved, which has made the code more readable and by extension more manageable. The major achievement in the new front end architecture is code reusability. By creating custom server controls using Object Oriented Programming concepts this front end design forms the standard for reusable code in CADEC. With a new design, expanding the features CADEC or implementing new chapters into CADEC has now been made easier for the developer. The developer can now invest more time in developing business logic or methods that implement newer procedures.

The web application that has been developed, adopts its structure from CADEC (CADEC Online, cadec-online.com) because of integration considerations. The software methods developed by (Saas, 2013) for design procedures have been placed in the 'Kernel' Project. Changes that have been made to implement them in the application are described in [appendix]. A new database has been created for the application which accommodates new entities. The presentation layer is designed as a three step process for each of the cases: Beam in Flexure, Beam in Shear, and Column Axial Loads. New step by step process is introduced to accommodate long design procedures. Also this step by step process lets the user navigate with ease to different step and analyze the model.

To validate the application, it is tested with examples of the designs mentioned in (Saas, 2013). The application produces same results when compared to windows application by (Saas, 2013) from which the software methods are adopted. 


\subsection{Future Work}

This sole purpose of developing this application is to make the chapter 13 'Strengthening of Reinforced Concrete' available at (CADEC Online, cadec-online.com). The present work accomplishes all the requirements to implement Chapter 13 abiding by (CADEC Online, cadeconline.com) architecture.

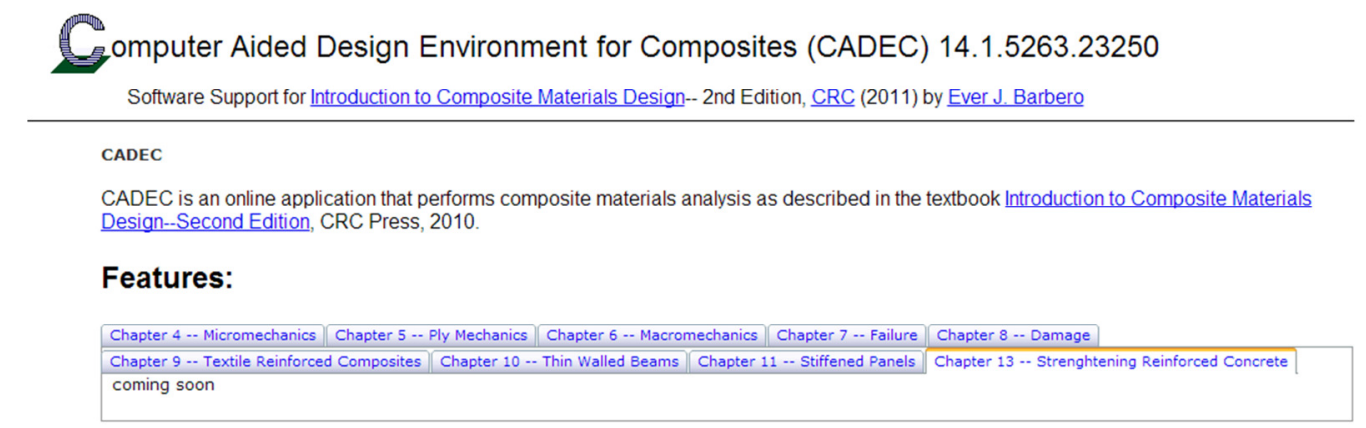

Figure 7-1: Picture of $\underline{w w w . c a d e c-o n l i n e . c o m}$ home page showing status of Chapter 13-

'Strengthening Reinforced Concrete'

Following section explains files those need to be added into CADEC.

Database: The database entities created in for the application are new and completely independent of the entities present in the CADEC database. Hence, all the entities and corresponding stored procedures have to be added to the CADEC database.

Kernel: Design procedures and classes that communicate with database present in 'Kernel' 'Strengthening of RC' application have to be added 'Kernel' project CADEC.

Web- Chapters: CADEC is organized in such a way that all the webpages that execute functionalities belonging to a chapter are put under corresponding section. So to add the new chapter, a section or folder has to be created for 'Strengthening of RC', to which all the webpages under Chapters in 'Strengthening of RC' application must be added.

Web- My Documents: The pages those perform adding, editing and deleting files from database are present under 'My Documents' in CADEC and also in 'Strengthening of RC' application. All the files from 'Strengthening of RC' application must be added to 'My Documents' in CADEC.

Web- Themes: Though styles in 'Strengthening of RC' application are inherited from CADEC, new style sheet 'Wizard.css' is defined for styles new control 'wizard' which shows the process step by step. Hence, this file should be added to 'Default Themes' in Web folder of CADEC. 


\section{Bibiligraphy}

ACI 318-08. (2008). Building Code Requirements for structural Concrete and Commentary. Farmington Hills, MI: American Concrete Institute.

ACI 440.2R-08. (2008). Guide for the Design and Construction of Externally Bonded FRP Systems for Strengthening Concrete Structures. Farmington Hills, MI: American Concrete Institute.

Bank, L. C. (2006). Composites for Construction: Structural Design with FRP Materials. John Wiley \& Sons.

Barbero, E. J. (2011). Introduction to Composite Material Design- 2nd edition. Boca Raton, FL: CRC Press.

(cadec-online.com). Retrieved from CADEC Online: www.cadec-online.com

Cosso, F. (2011). Diseño Asistido por Computadora de Materiales Compuestos. UNIVERSIDAD NACIONAL DE GENERAL SAN MARTÍN .

Hota, G. V., Taly, N., \& P.V., V. (2006). Reinforced Concrete Design with FRP Composites. Taylor \& Francis.

Kothari, N., \& Datye, V. (2003). ASP.NET Server Controls and Components. Microsoft Press.

Saas, T. D. (2013). Design Software Methods for Strengthening of Reinforced Concrete Beams and Columns. West Virginia University.

Computer Aided Design Environment for Composites (CADEC): www.cadec-online.com 


\section{Appendix A}

\section{Microsoft SQL Scripts}

Database Table: CADEC_RCMemberConfiguration

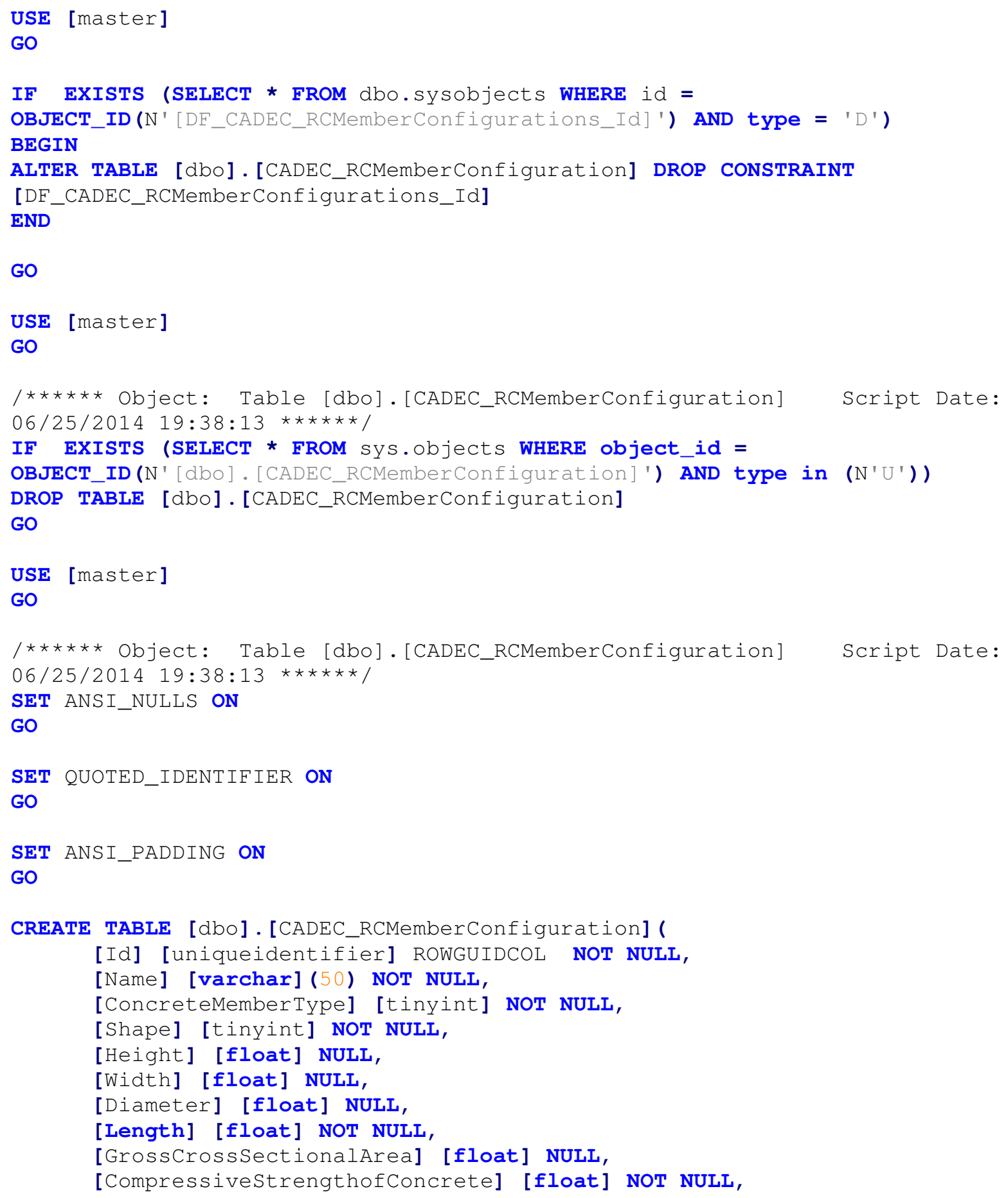




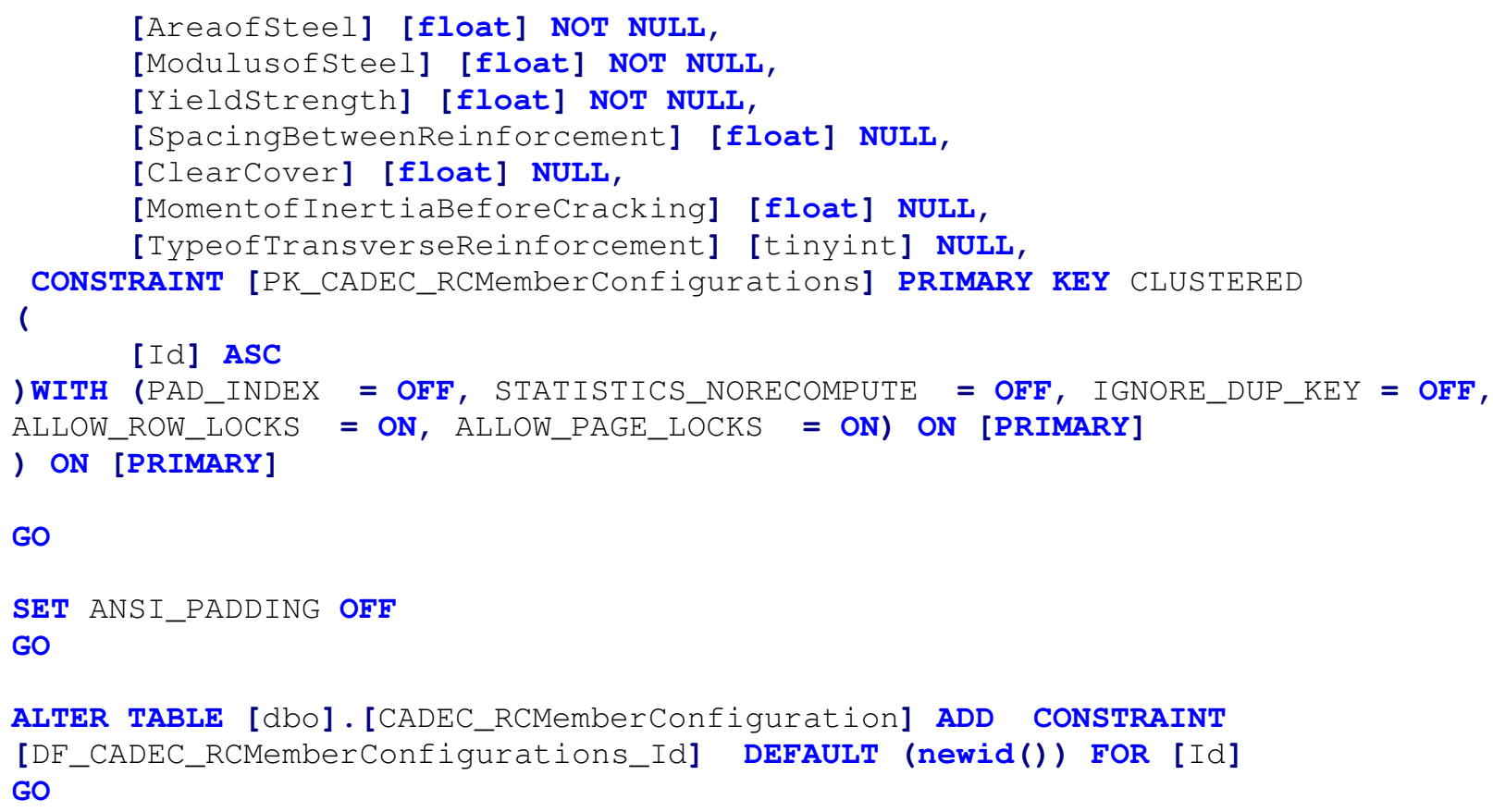

Database Table: CADEC_RCMemberLoads

USE [master]

GO

IF EXISTS (SELECT * FROM dbo.sysobjects WHERE id = 


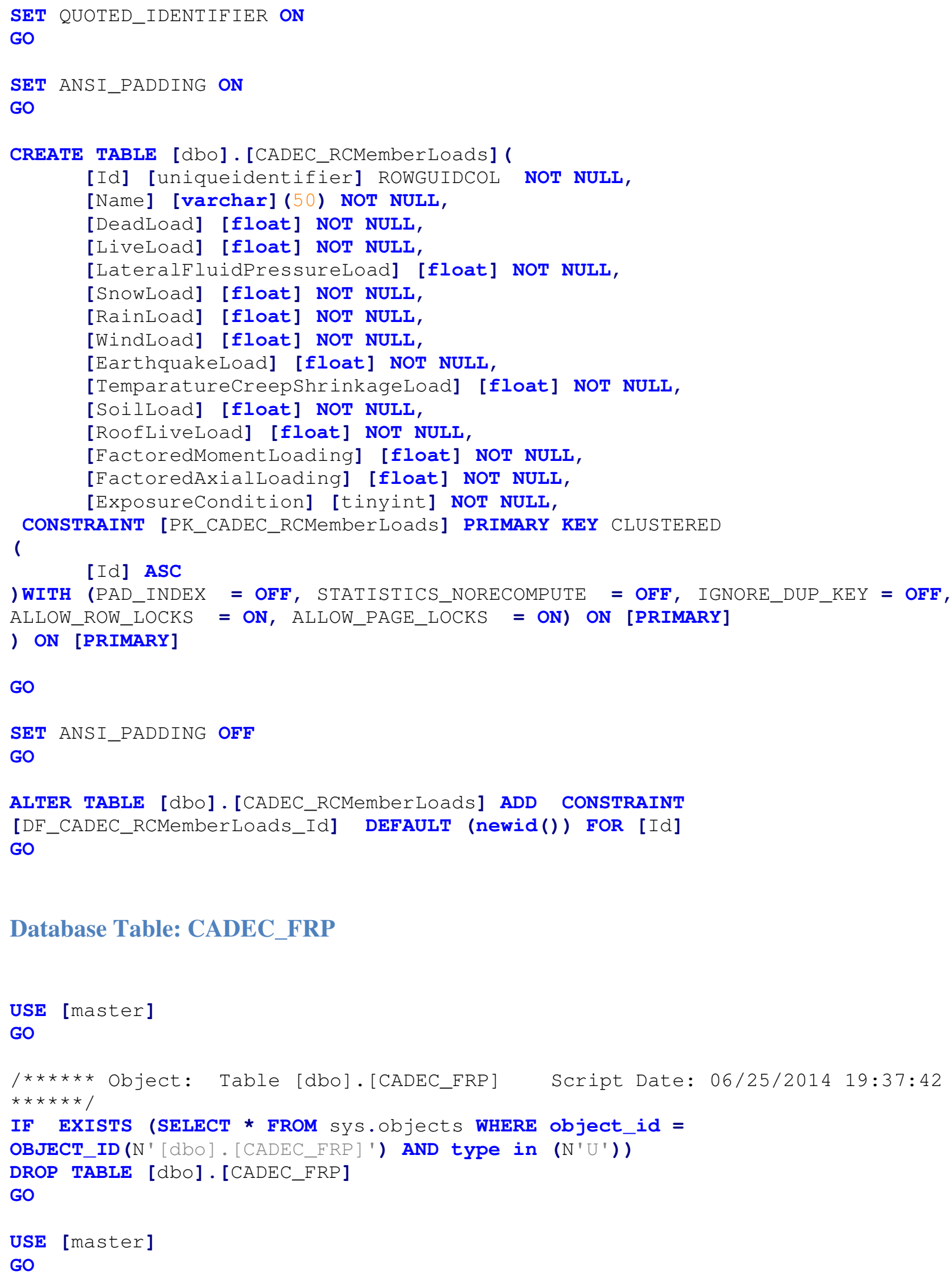




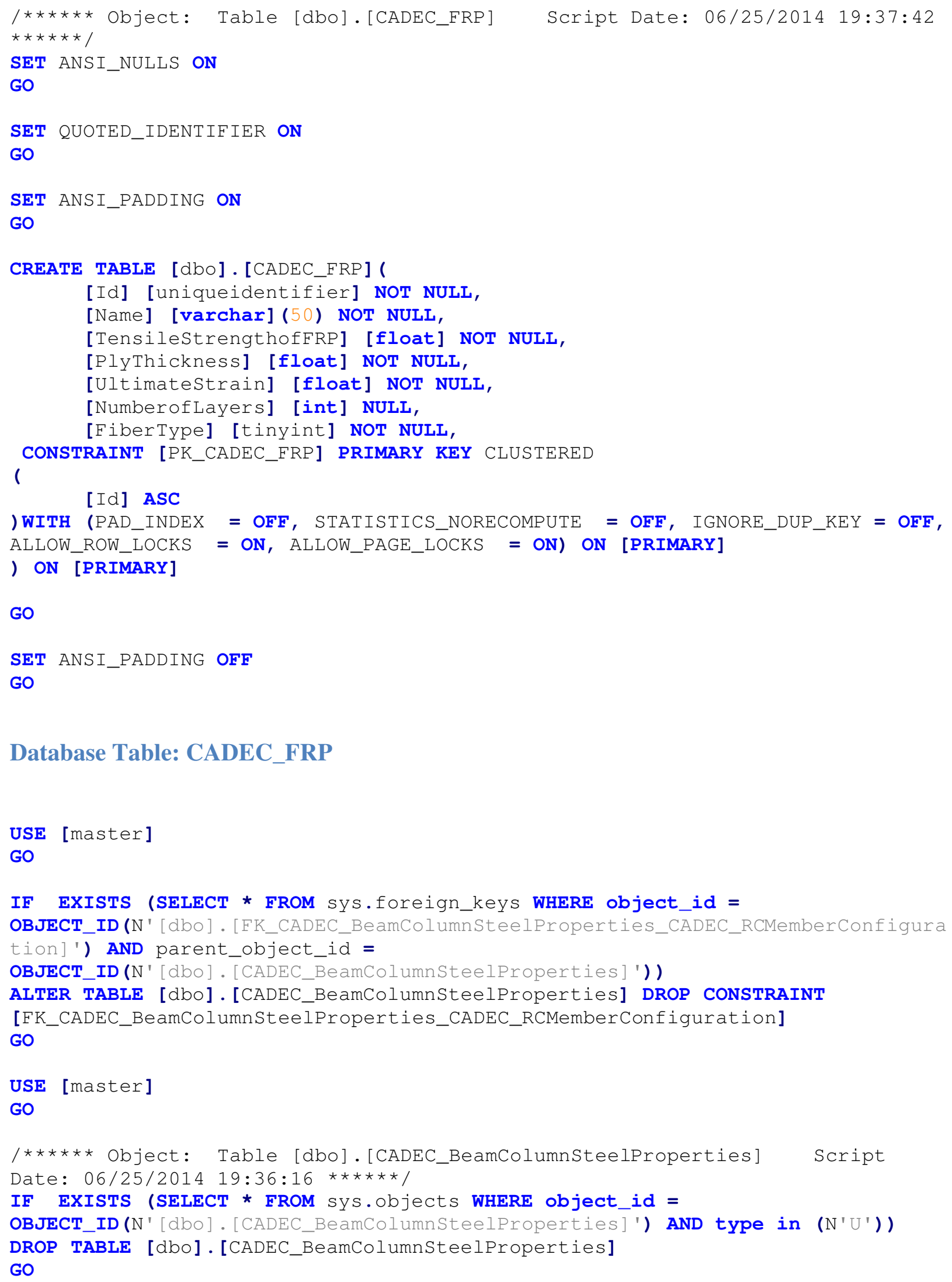




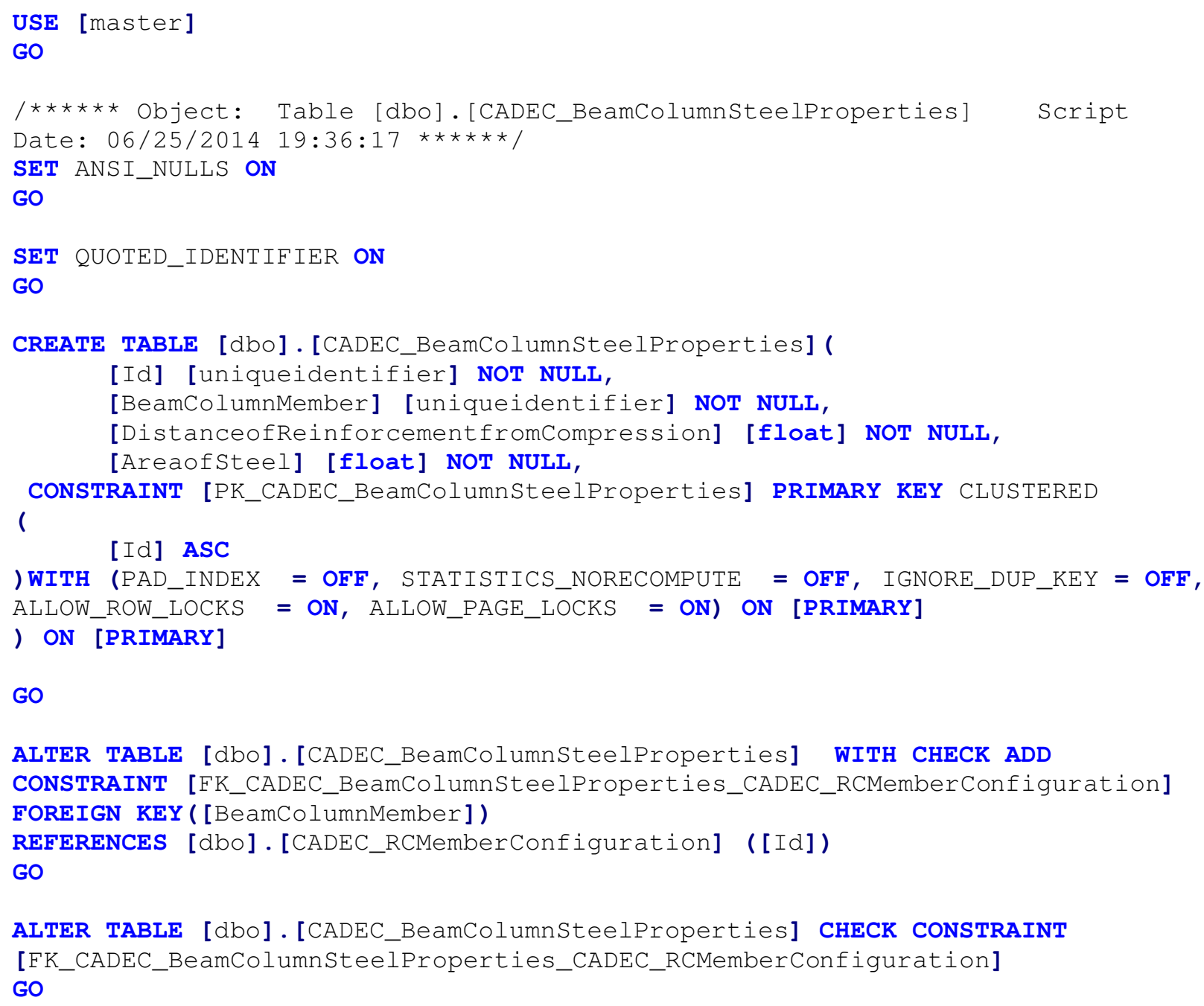


GO

SET QUOTED_IDENTIFIER ON

GO

-- Batch submitted through debugger:

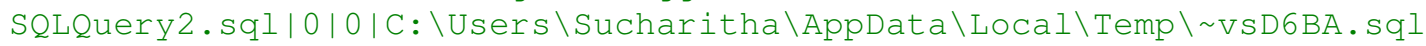

CREATE PROCEDURE [dbo].[CADEC_RCMemberConfiguration_Insert]

(ad uniqueidentifier output,

@Name varchar (50),

@ConcreteMemberType tinyint,

eShape tinyint,

@Height float,

QWidth float,

QDiameter float,

eLength float,

@GrossCrossSectionalArea float,

@CompressiveStrengthofConcrete float,

@Areaofsteel float,

@Modulusofsteel float,

QYieldStrength float,

aSpacingBetweenReinforcement float,

@ClearCover float,

@MomentofInertiaBeforeCracking float,

@TypeoftransverseReinforcement tinyint

AS

SELECT @Id = NEWID ()

INSERT INTO CADEC_RCMemberConfiguration

(Id, Name, ConcreteMemberType, Shape, Height, Width,

Diameter, Length, GrosscrossSectionalArea, CompressivestrengthofConcrete,

Areaofsteel, Modulusofsteel, YieldStrength,

SpacingBetweenReinforcement, ClearCover, MomentofInertiaBeforeCracking, TypeofTransverseReinforcement) )

VALUES (@Id, @Name, @ConcreteMemberType, @Shape, @Height, @Width, eDiameter, elength,

@GrosscrosssectionalArea, @CompressivestrengthofConcrete, @Areaofsteel, aModulusofsteel,

@YieldStrength, @SpacingBetweenReinforcement, @clearcover,

aMomentofInertiaBeforeCracking,

@TypeoftransverseReinforcement )

RETURN

GO

Stored Procedure: CADEC_RCMemberConfiguration_Update

USE [master]

GO

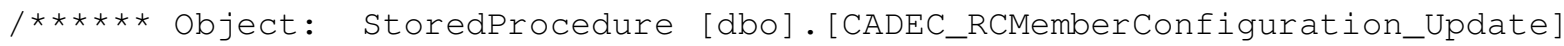

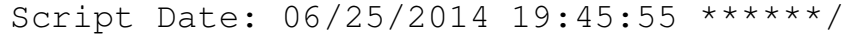




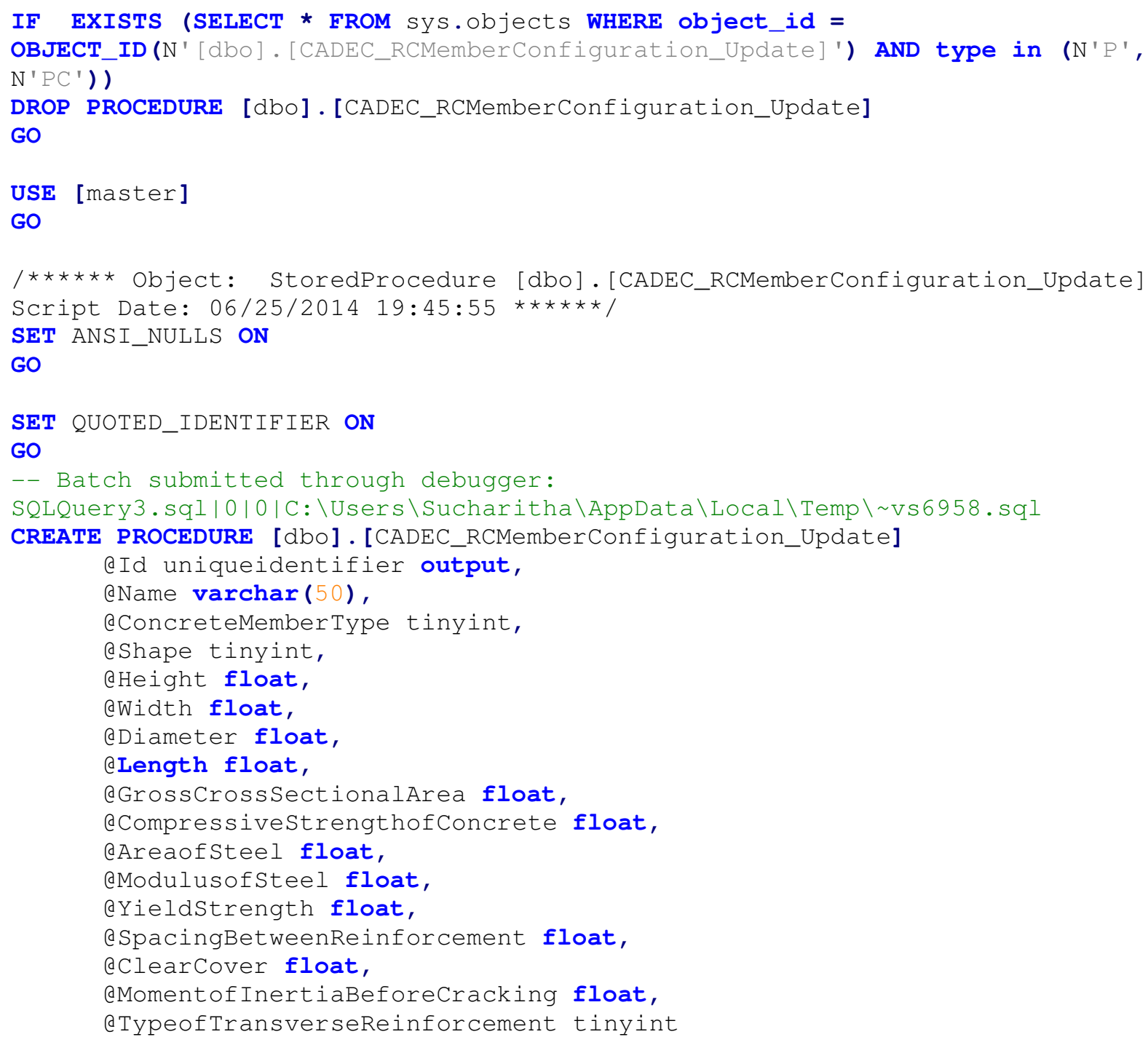

AS

UPDATE CADEC_RCMemberConfiguration

SET Name=@Name,

ConcreteMemberType=@ConcreteMemberType,

Shape=@Shape,

Height=@Height,

Width=@Width,

Diameter=@Diameter,

Length $=@$ Length,

GrossCross SectionalArea=@Grosscross SectionalArea, Compressivestrengthof Concrete= CompressivestrengthofConcrete, Areaofsteel=@Areaof Steel, Modulusof Steel=@Modulusofsteel, YieldStrength=@YieldStrength, SpacingBetweenReinforcement=@SpacingBetweenReinforcement, ClearCover $=$ clearCover, MomentofInertiaBeforeCracking=@Momentof InertiaBeforeCracking, TypeofTransverseReinforcement=@TypeofTransverseReinforcement $(I d=(I d)$ 


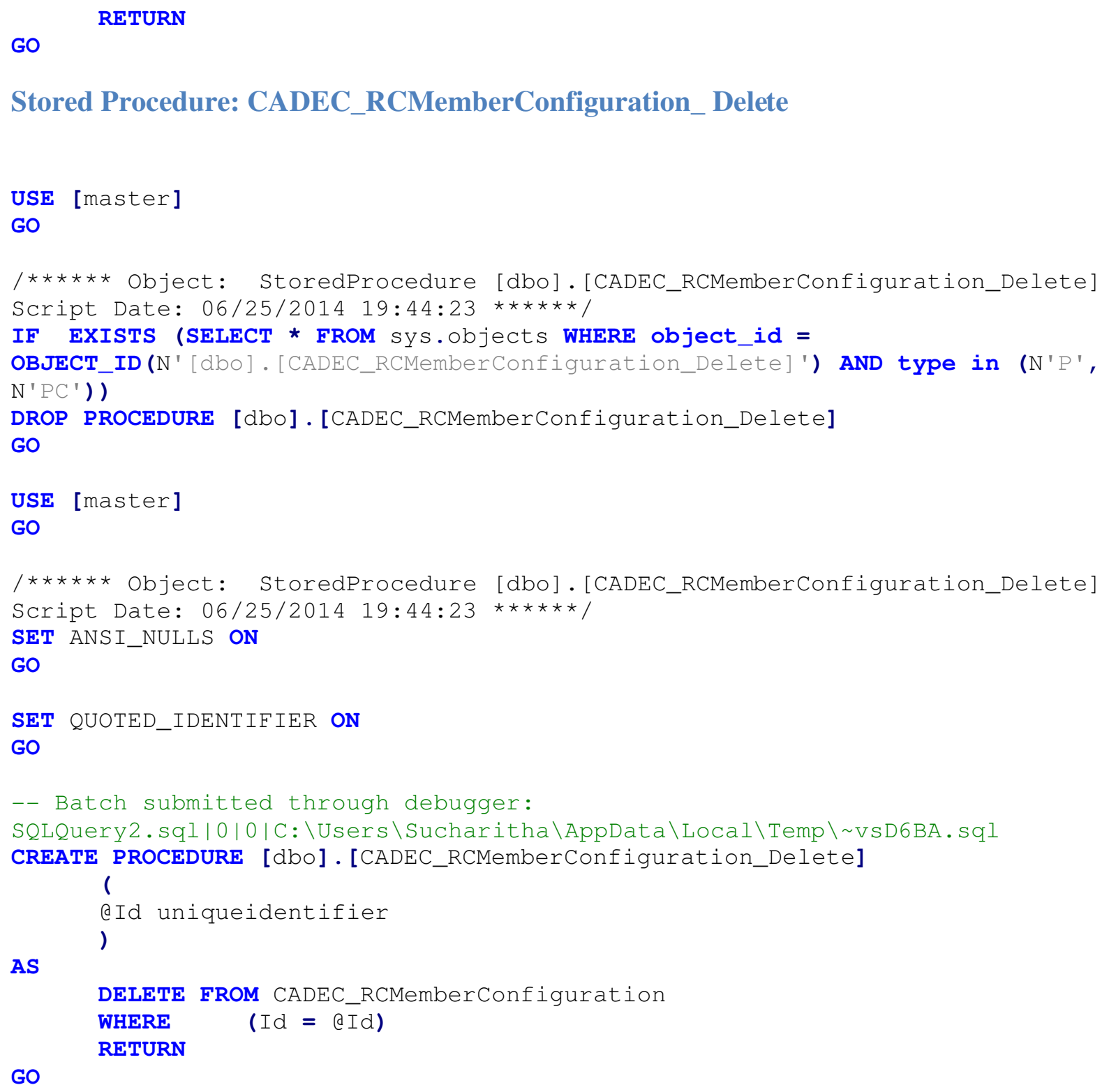


GO

/****** Object: StoredProcedure [dbo].[CADEC_RCMemberConfiguration_Get_One]

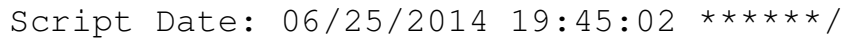

SET ANSI_NULLS ON

GO

SET QUOTED_IDENTIFIER ON

GO

-- Batch submitted through debugger:

SQLQuery2 .sql | 0 | 0 | C: \Users \Sucharitha \AppData \Local \Temp \ vsD6BA.sql

CREATE PROCEDURE [dbo].[CADEC_RCMemberConfiguration_Get_One]

(

(a) uniqueidentifier

)

AS

SELECT Id, Name, ConcreteMemberType, Shape, Height, Width, Diameter, Length, GrossCrossSectionalArea, CompressivestrengthofConcrete, Areaofsteel, ModulusofSteel, YieldStrength, SpacingBetweenReinforcement, ClearCover, MomentofInertiaBeforeCracking, TypeofTransverseReinforcement

FROM CADEC_RCMemberConfiguration

WHERE (Id $=$ (Id)

RETURN

GO

Stored Procedure: CADEC_RCMemberConfiguration_Get_One

USE [master]

GO

/****** Object: StoredProcedure [dbo].[CADEC_RCMemberConfiguration_All] Script Date: 06/25/2014 19:43:48******/

IF EXISTS (SELECT * FROM sys.objects WHERE object_id =

OBJECT_ID(N'[dbo].[CADEC_RCMemberConfiguration_All]') AND type in (N'P', $\left.\mathrm{N}^{\prime} \mathrm{P} \mathrm{C}^{\prime}\right)$ )

DROP PROCEDURE [dbo].[CADEC_RCMemberConfiguration_All]

GO

USE [master]

GO

/****** Object: StoredProcedure [dbo].[CADEC_RCMemberConfiguration_All] Script Date: 06/25/2014 19:43:48******/

SET ANSI_NULLS ON

GO

SET QUOTED_IDENTIFIER ON

GO 
CREATE PROCEDURE [dbo].[CADEC_RCMemberConfiguration_All]

AS

SELECT Id, Name, ConcreteMemberType, Shape, Height, Width, Diameter, Length, GrosscrossSectionalArea, CompressiveStrengthofConcrete, AreaofSteel, ModulusofSteel, YieldStrength, SpacingBetweenReinforcement, ClearCover, MomentofInertiaBeforeCracking, TypeofTransverseReinforcement

FROM CADEC_RCMemberConfiguration

RETURN

GO

Stored Procedure: CADEC_RCMemberConfiguration_Get_One

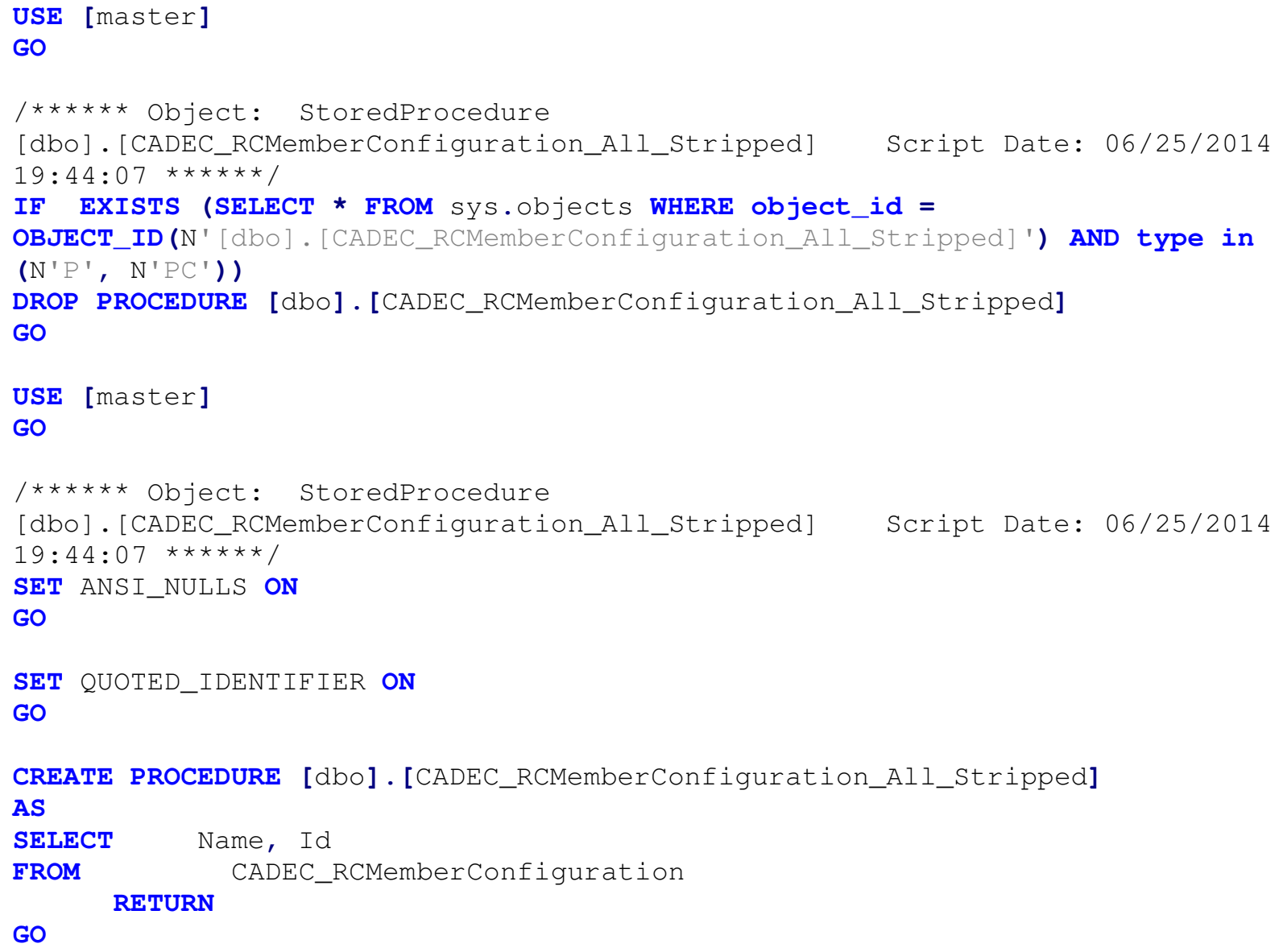




\section{Appendix B}

\section{Software installation.}

a. Software Required

- Microsoft SQL Server 2008, Express Edition

- Microsoft SQL Server 2008 SP4, Express Edition

- Microsoft SQL Server management Studio 2008, Express Edition

b. Installation Procedure for Microsoft SQL Server 2008

Following are step by step instructions to install database provider, which is necessary to run Web app.

- Download executable files following below links

http://www.microsoft.com/en-us/download/details.aspx?id=1695

http://www.microsoft.com/en-us/download/details.aspx?id=44278

- Run the file SQLEXPR_x64_ENU.exe

- Click on Installation from the menu on left side of the window as shown.

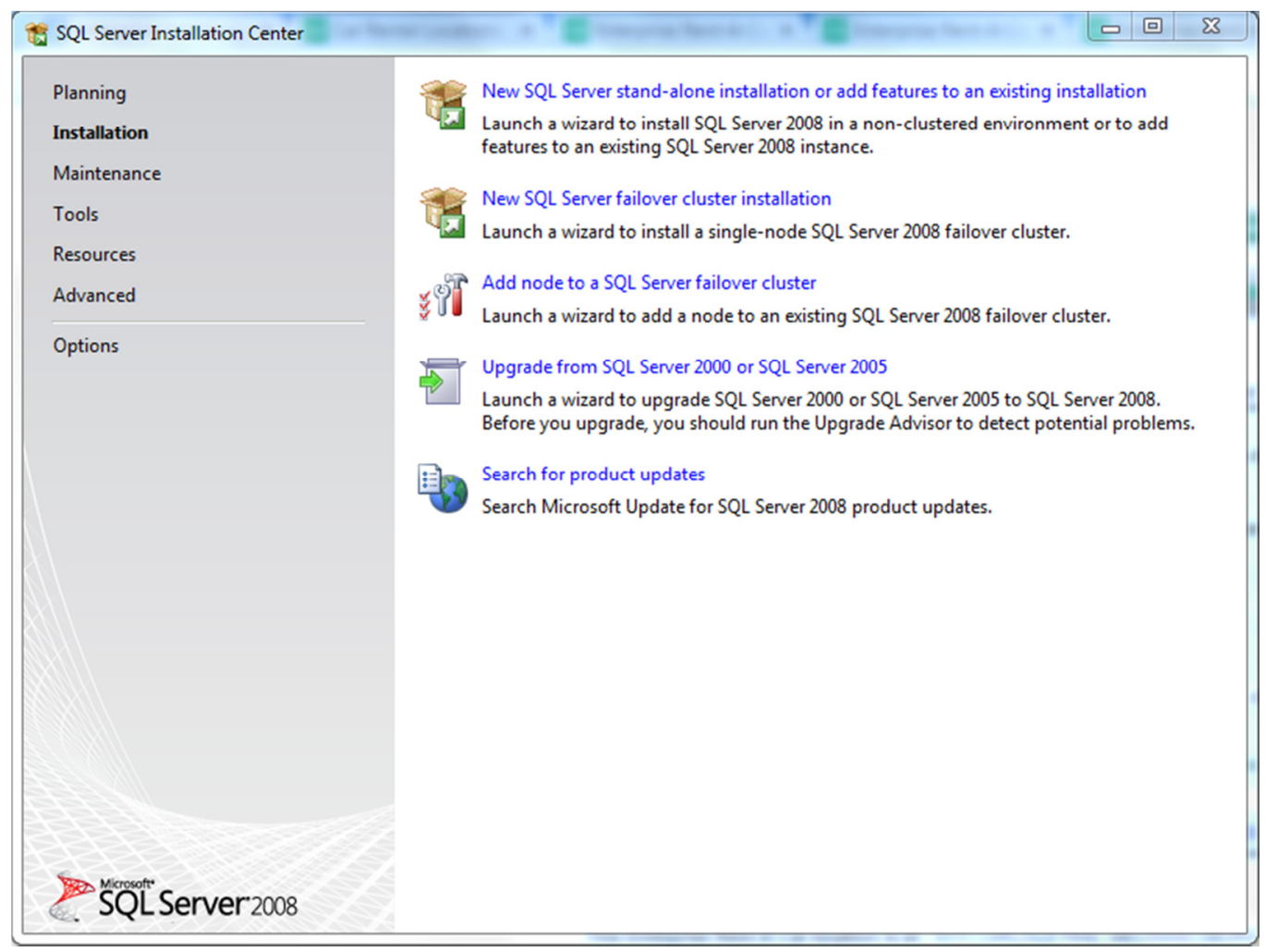

- Then select the first option from the list that appears on left side that says 'New SQL Server stand-alone installation or add features to an existing installation'. 
- It is followed by check for setup support as shown. Click on 'OK' to install Setup Support.

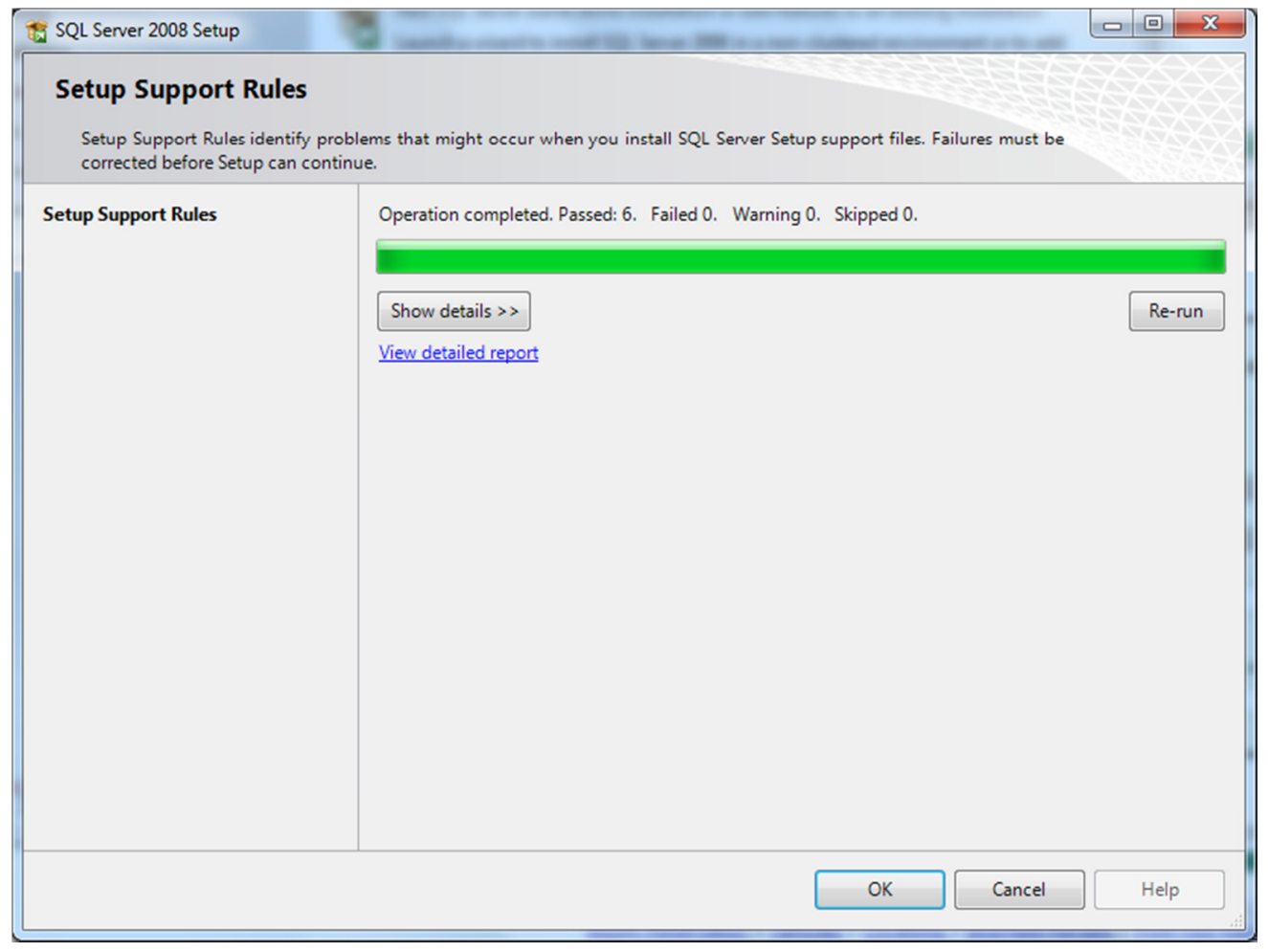

- Click on 'Install' and wait till installation completes successfully. After Completion click 'Next'

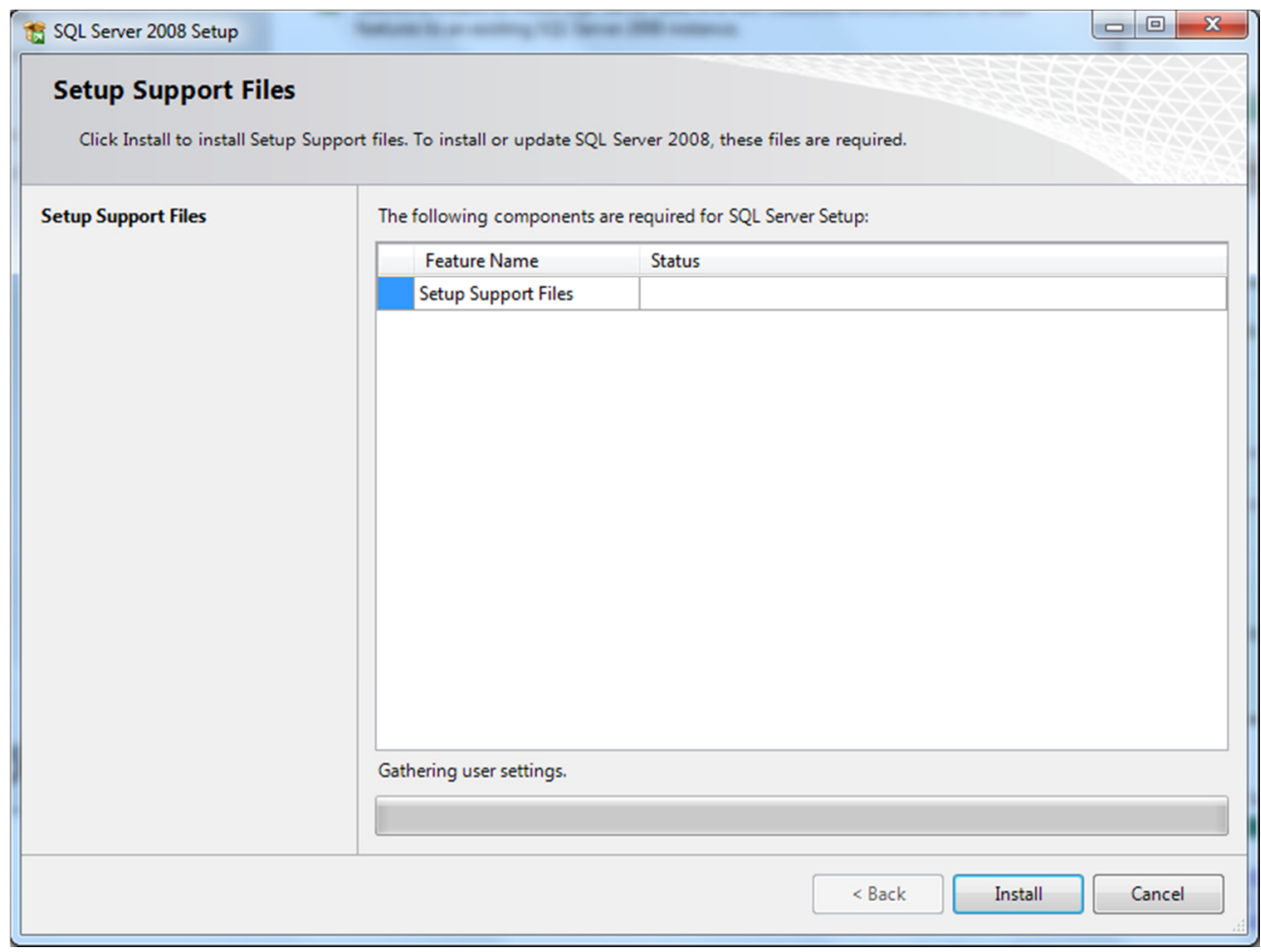




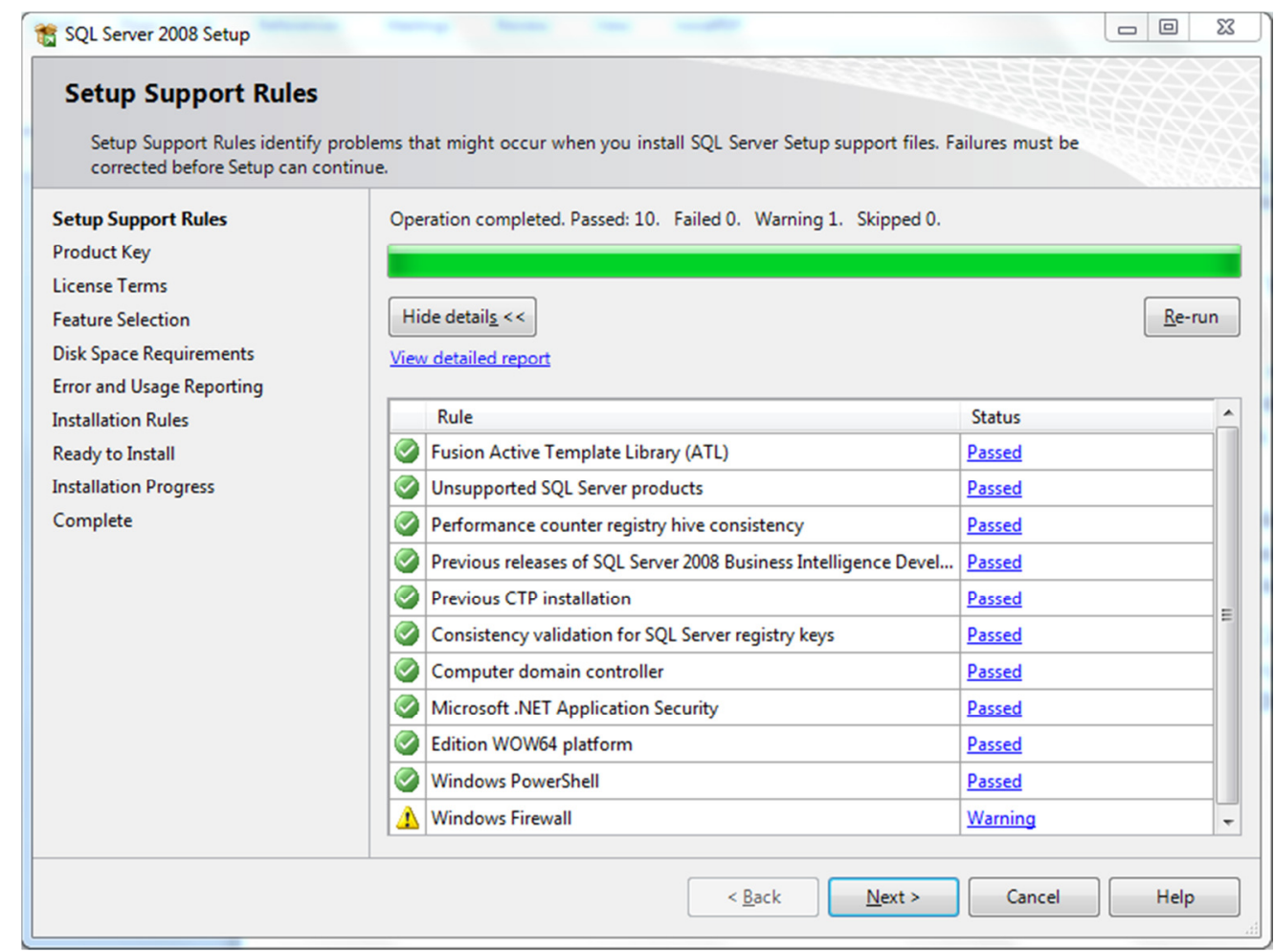

- Since express edition is freeware provided by Microsoft, no product key is required.

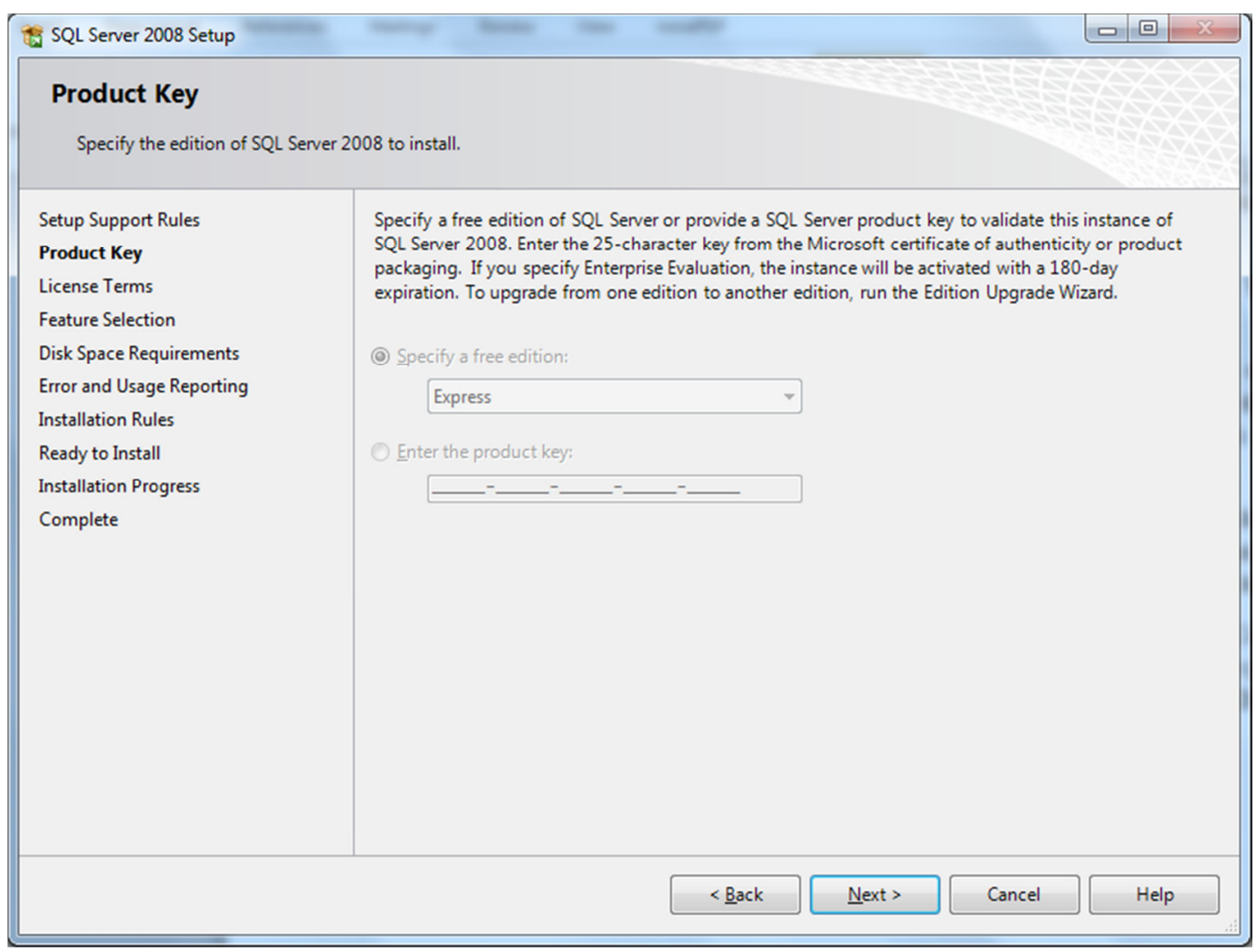


- Accept License Terms and click 'Next' to proceed.

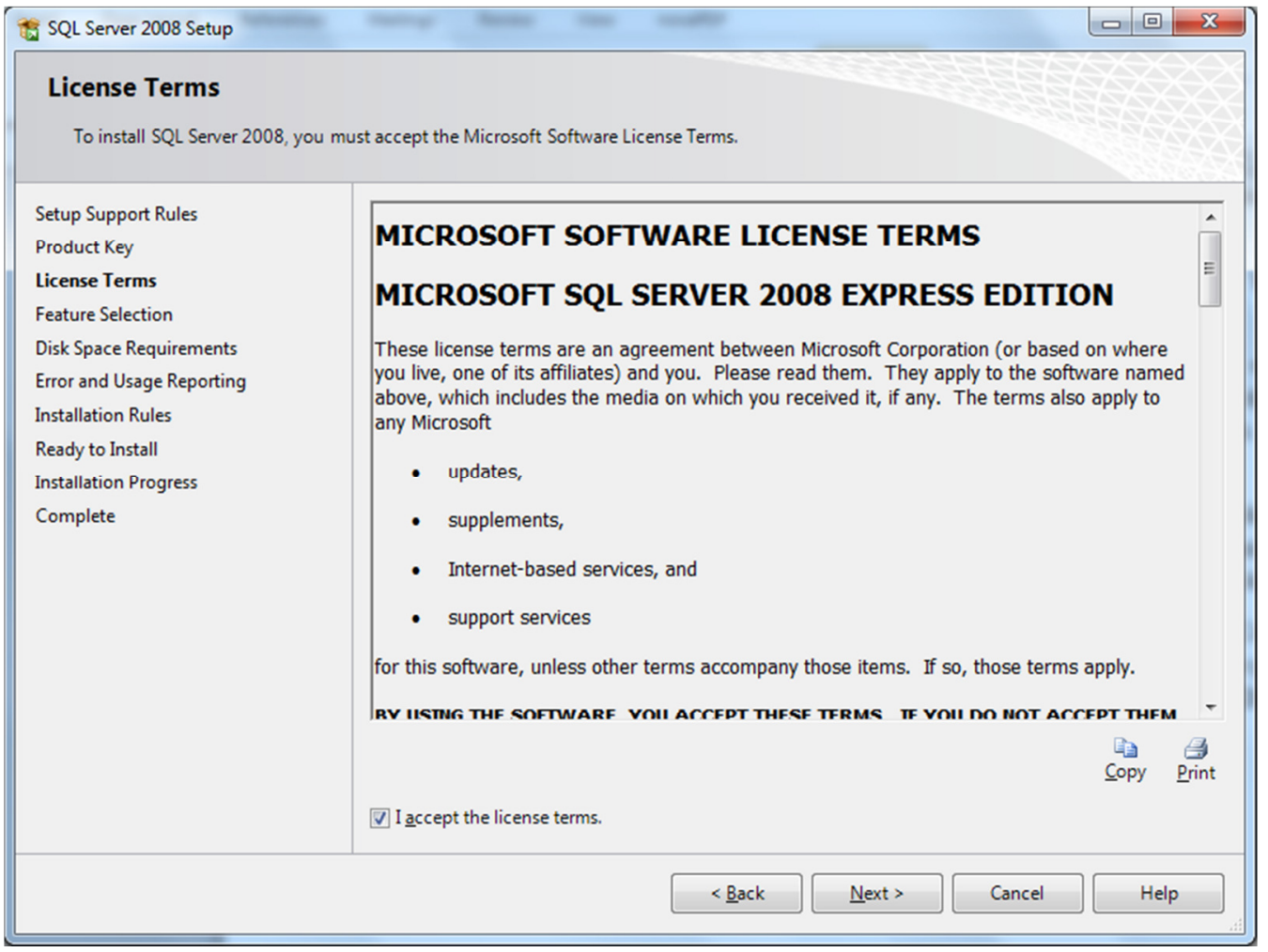

- In Feature Selection, select all the features. Click 'Next' to proceed.

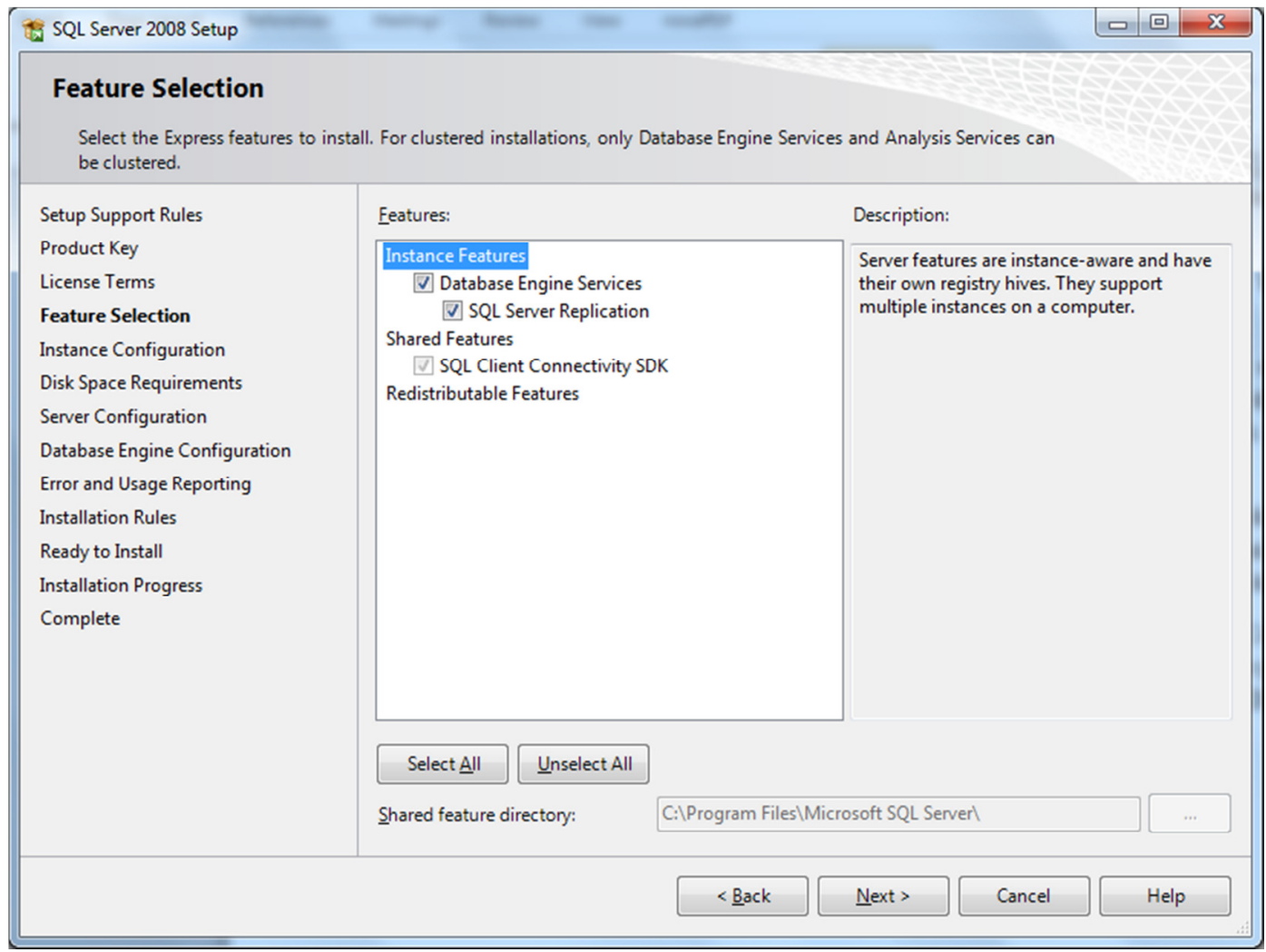


- In Instance Configuration, one can either choose default instance or can create new instance. Instance is a set of memory that is used as database. New database can be created by installing new instance. List of existing databases can be seen under 'Installed instances'. Database directory can also be chosen from 'Instance root Directory'. Enter preferences and click next to proceed.

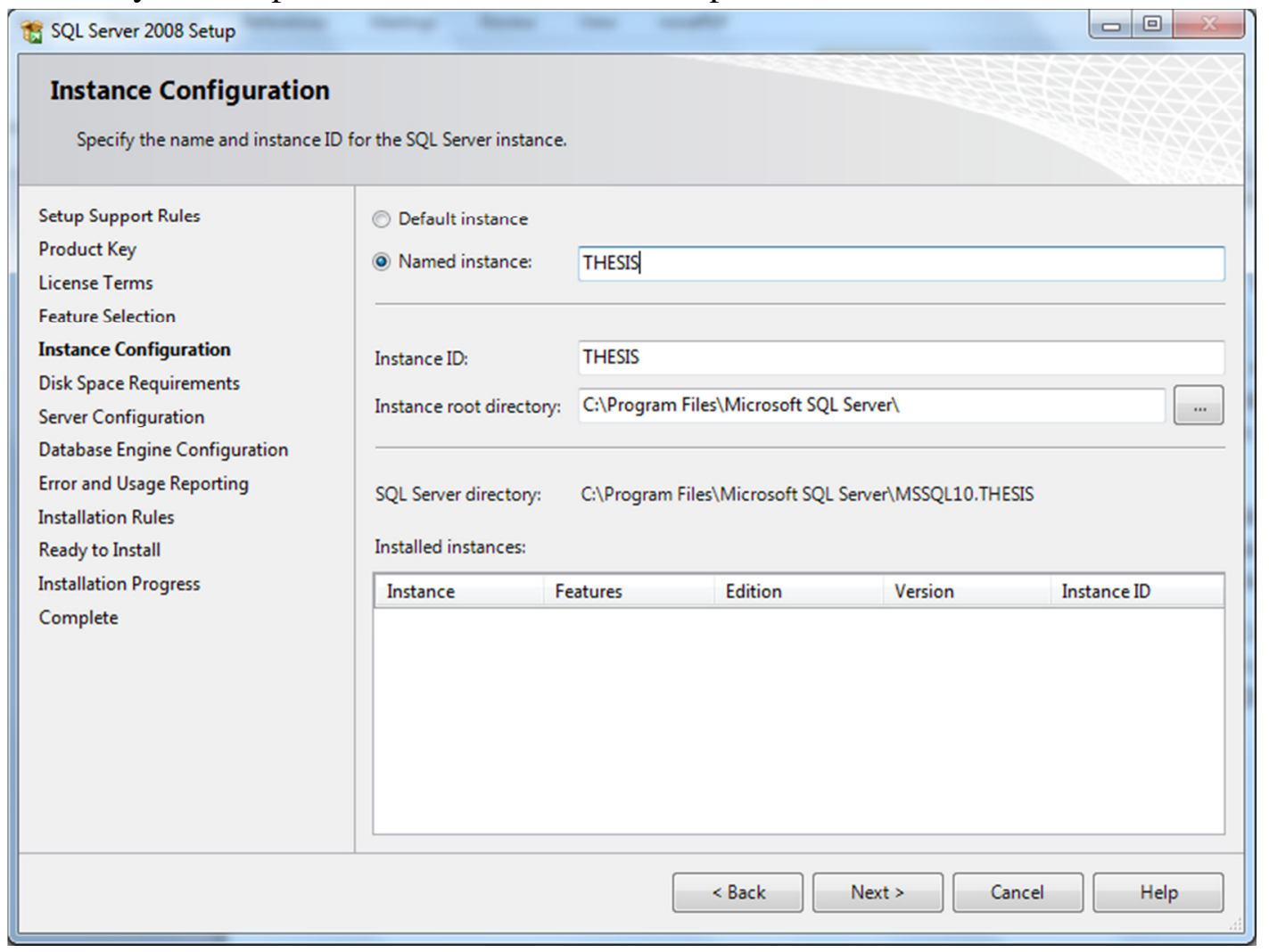


- Check for required disk space. If disk space is not available, press 'Back' and change the respective directory location.

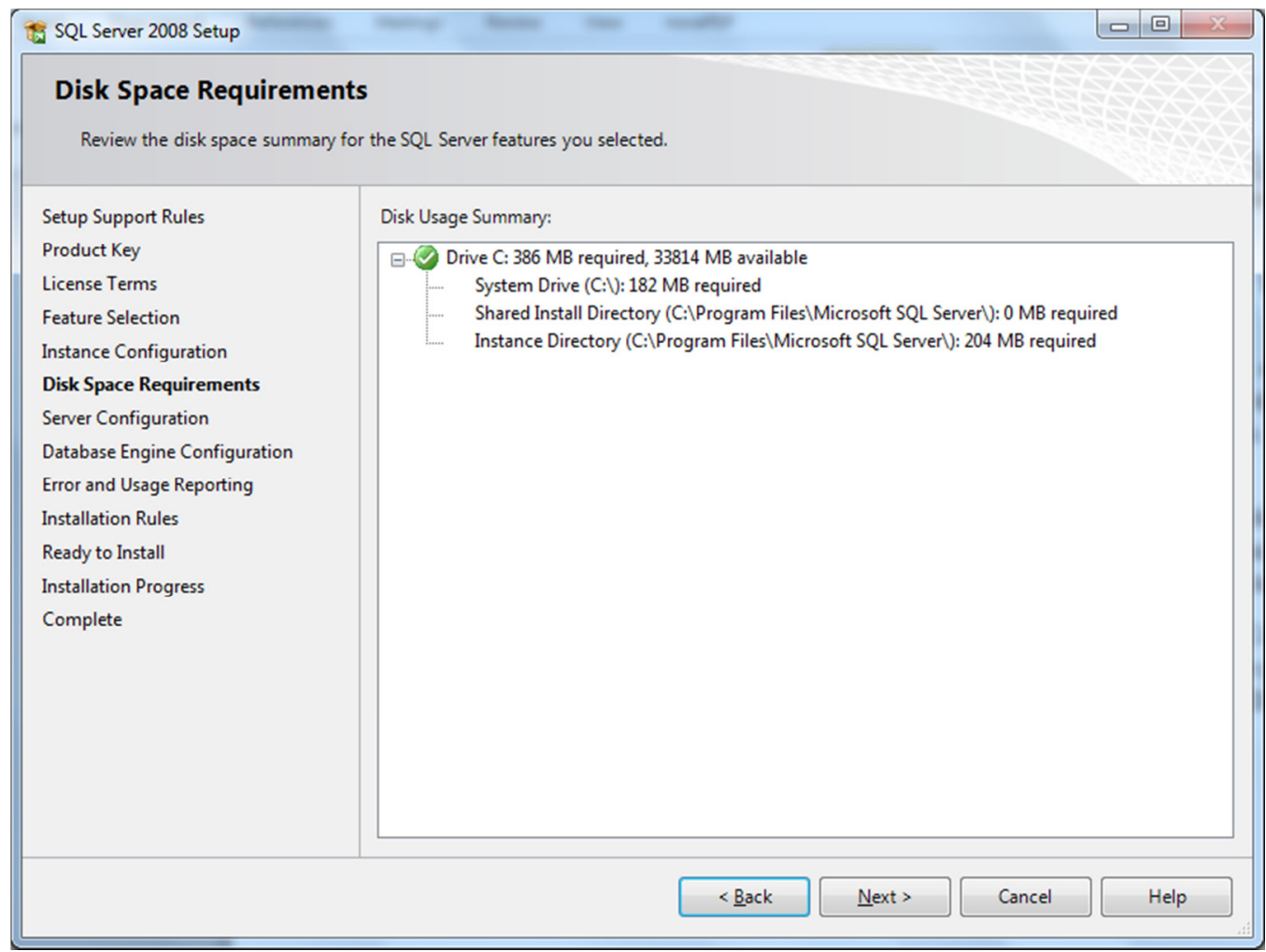


- In Sever Configuration step, configure both SQL Server Database Engine and SQL Server Browser carefully. Choose 'NT AuthoritylSystem' for SQL Server Database Engine and 'NT AuthoritylLocal' for SQL Server Browser. Choose start up type 'Automatic' for both services.

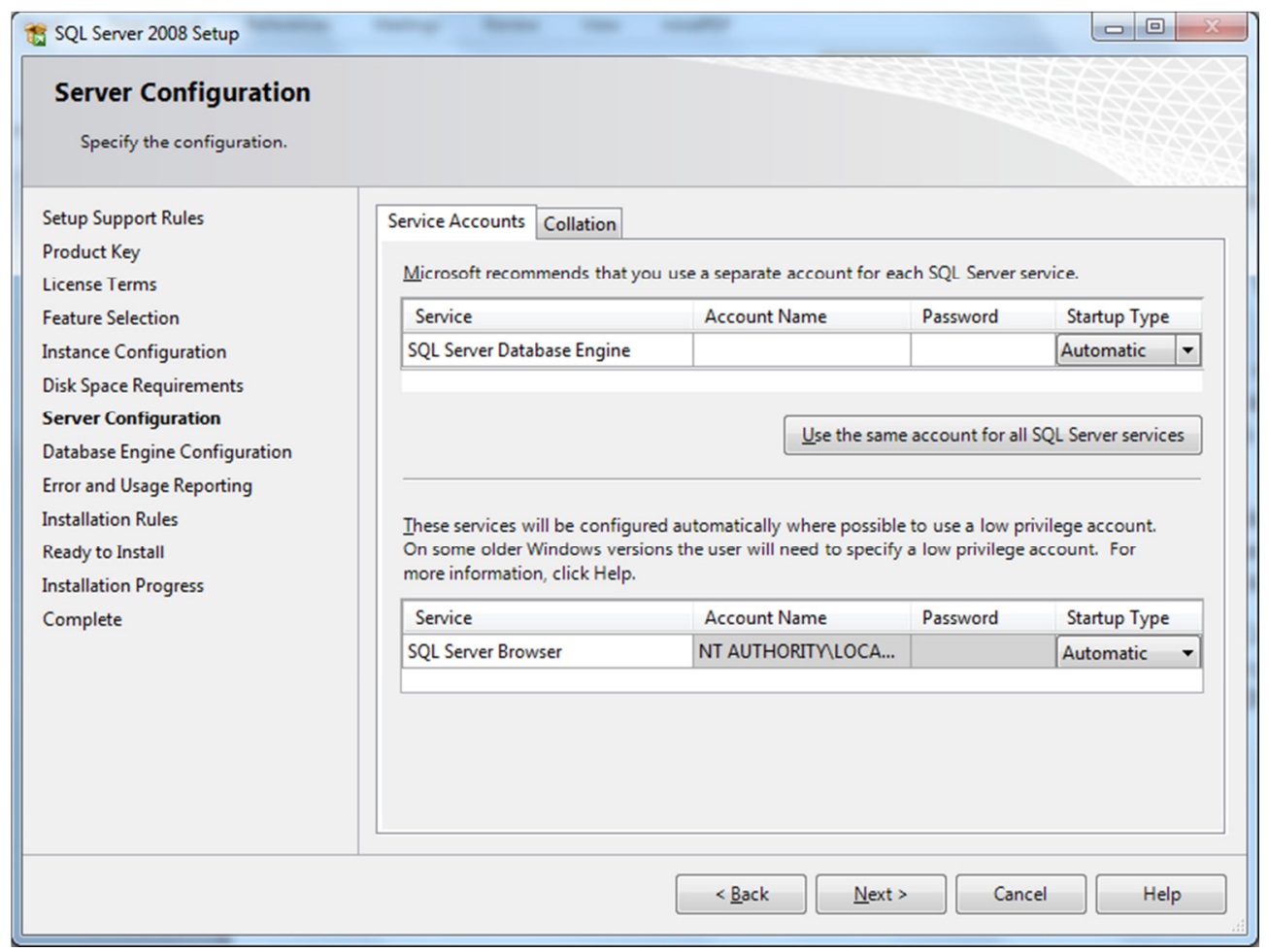

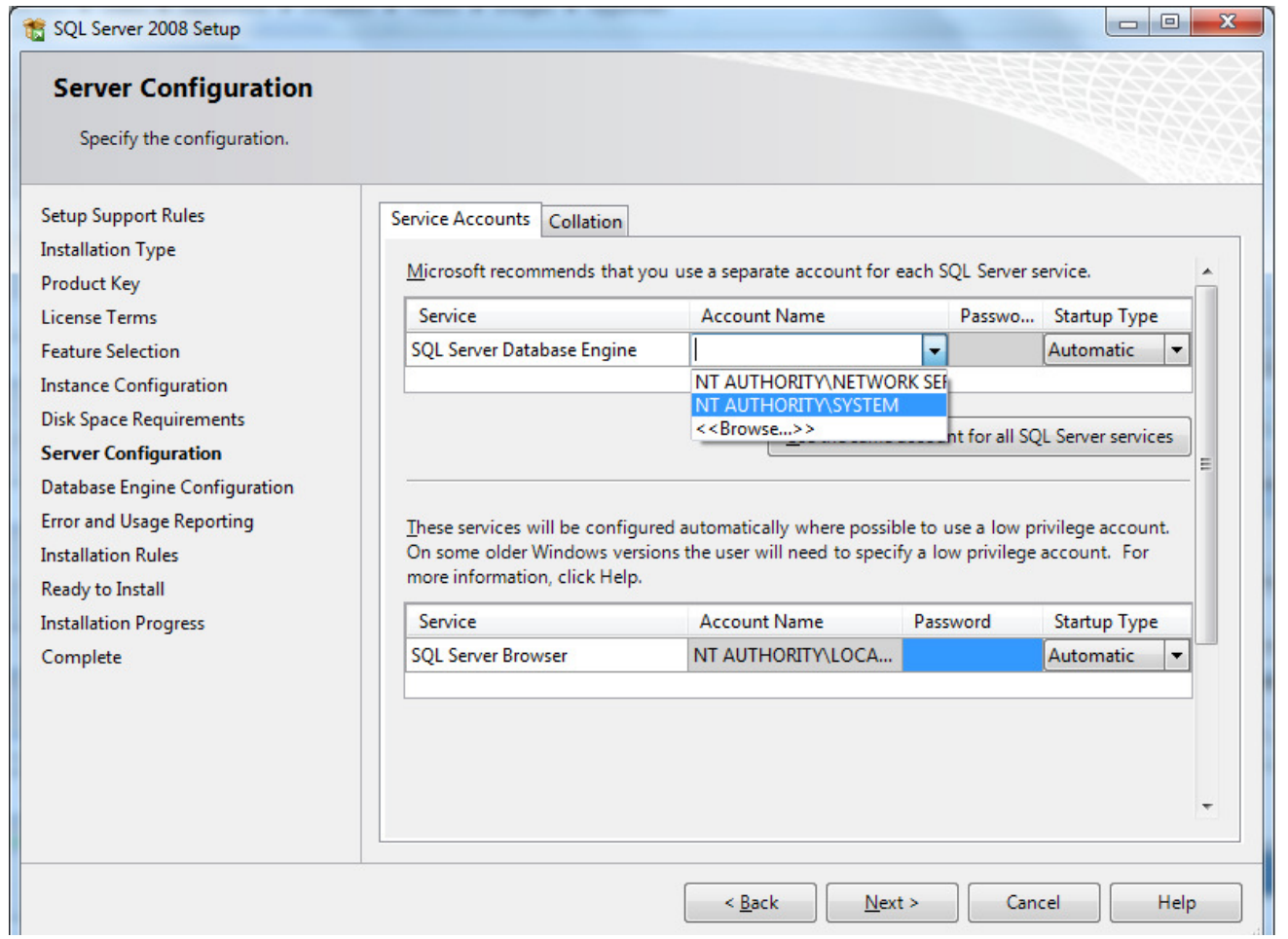


- In Database Engine Configuration step choose the type of authentication required and add all the users that should be given access. In this instruction only current user is given access.
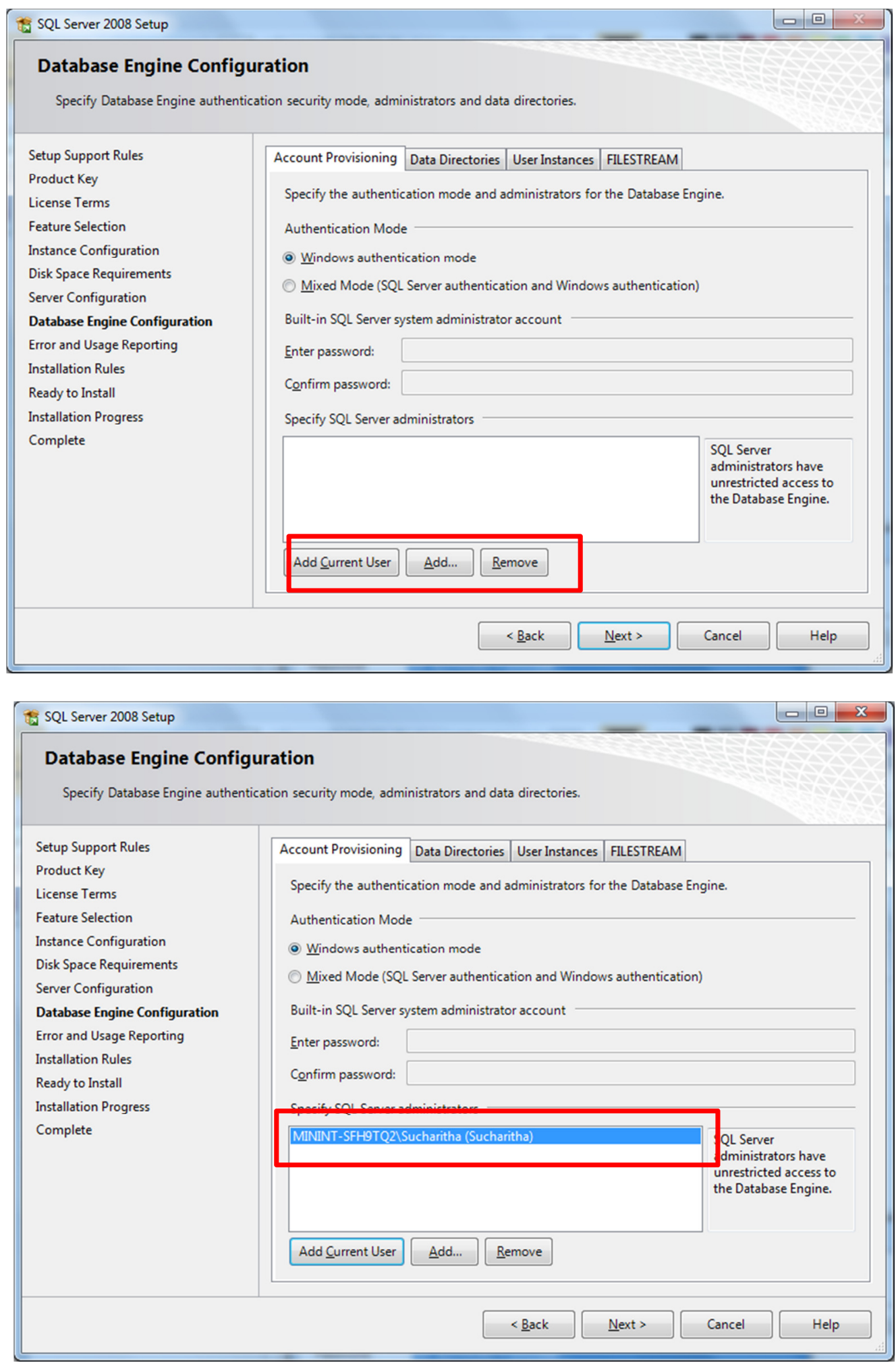
- Choose error reporting preferences in next step. Click next to proceed.

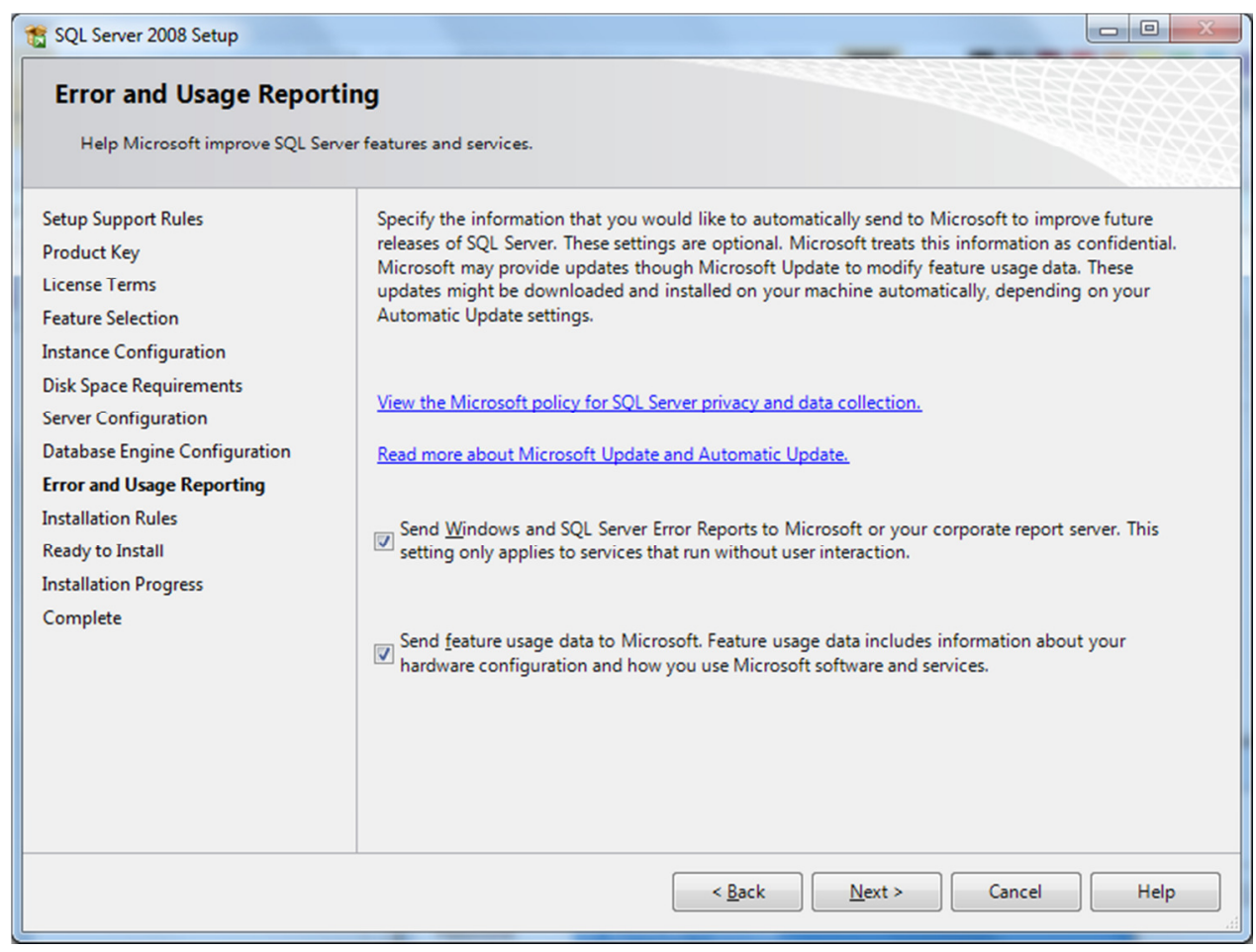

- Software checks for installation requirements here. After completion, click next to install.

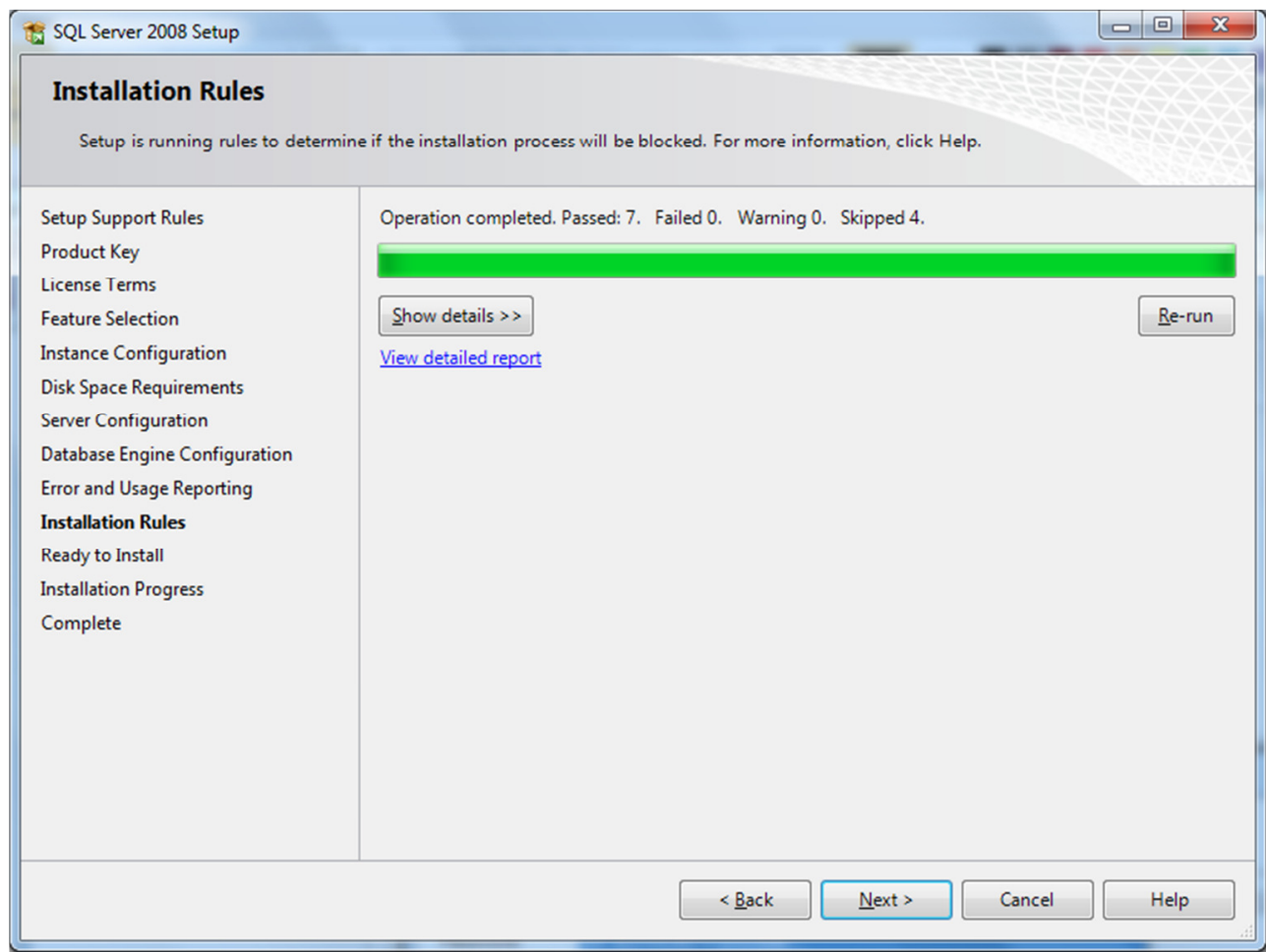


- Click on install.

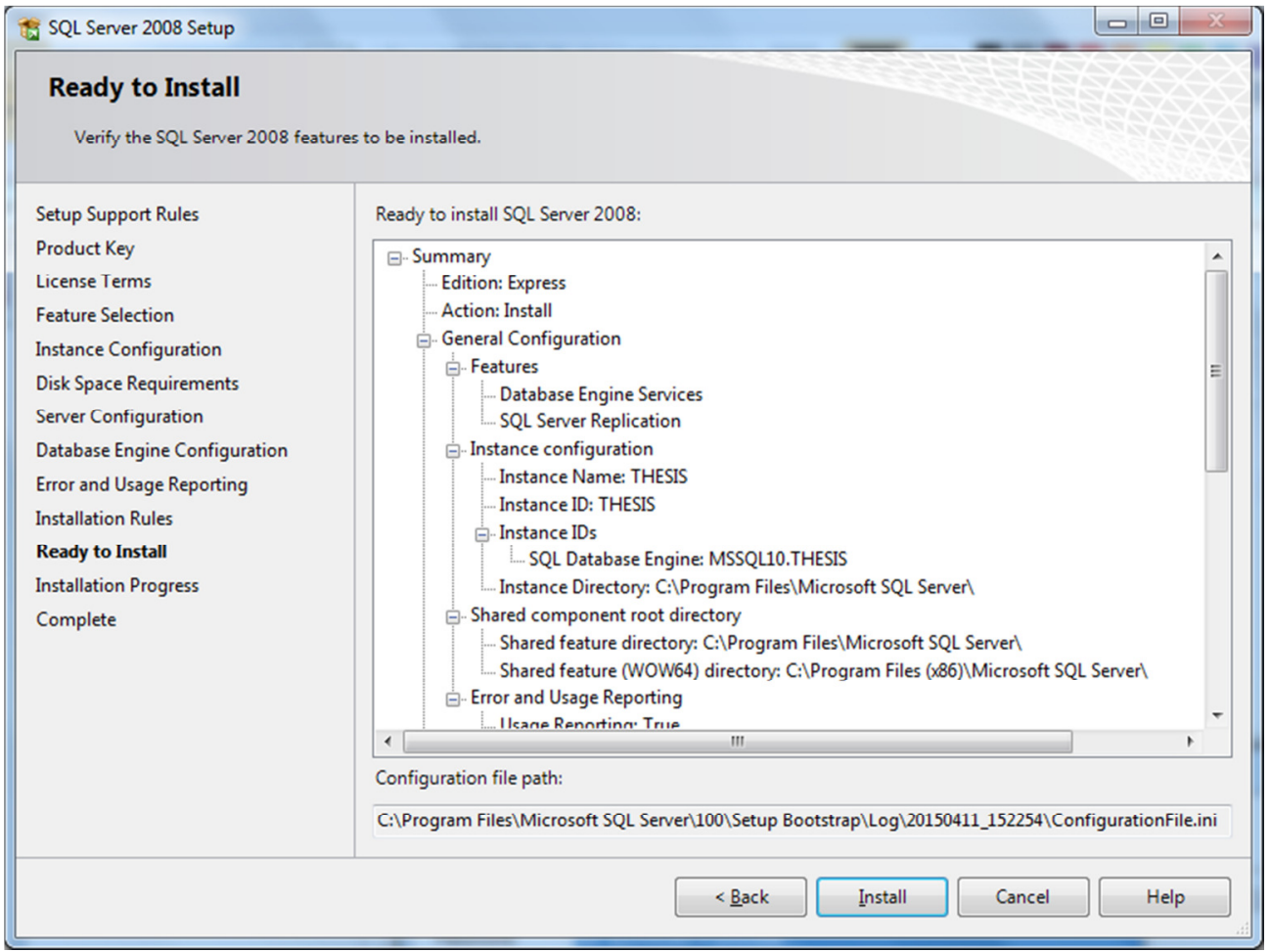

\section{- Wait for set to complete.}

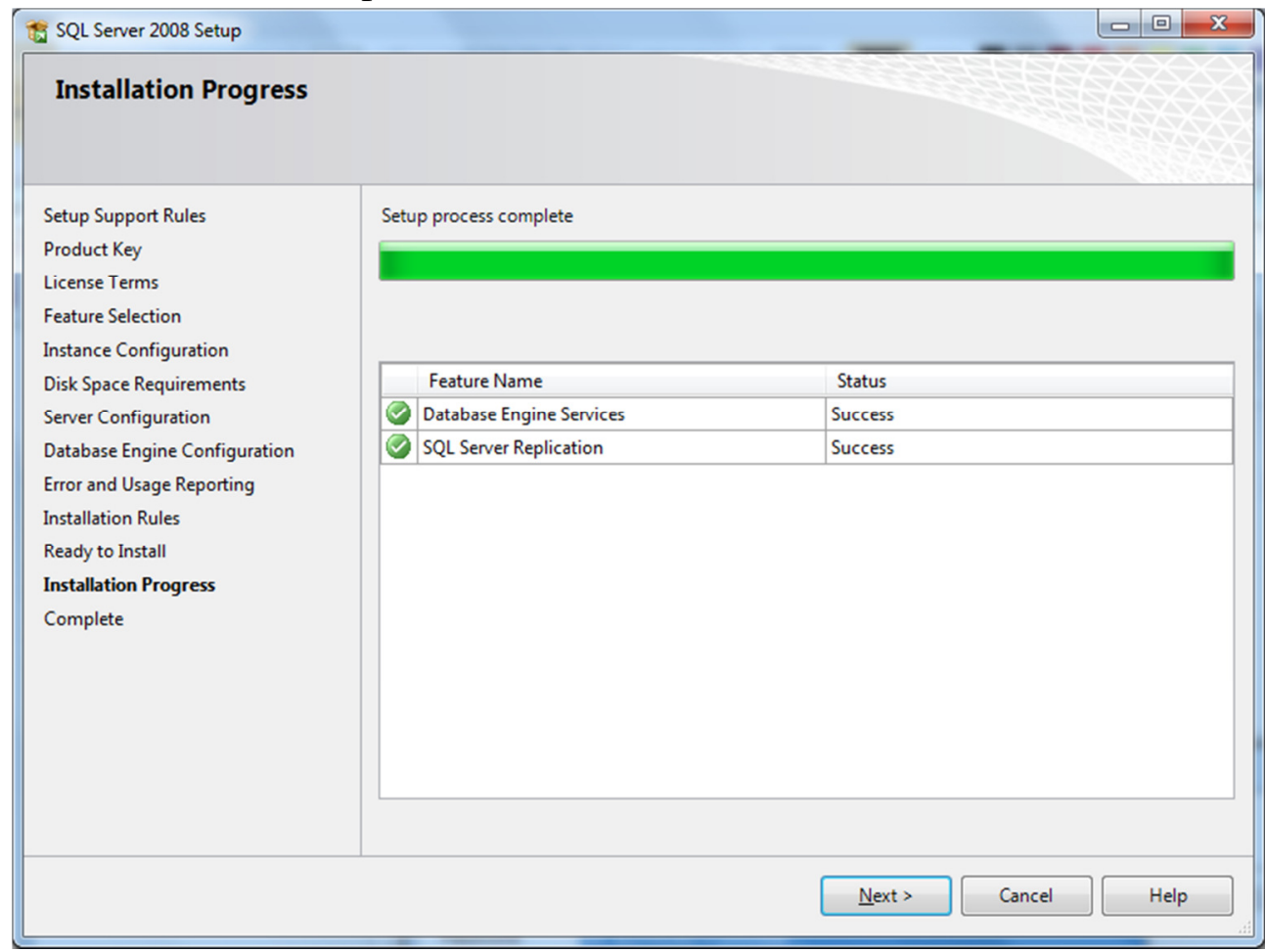


- Check for completion message in last step.

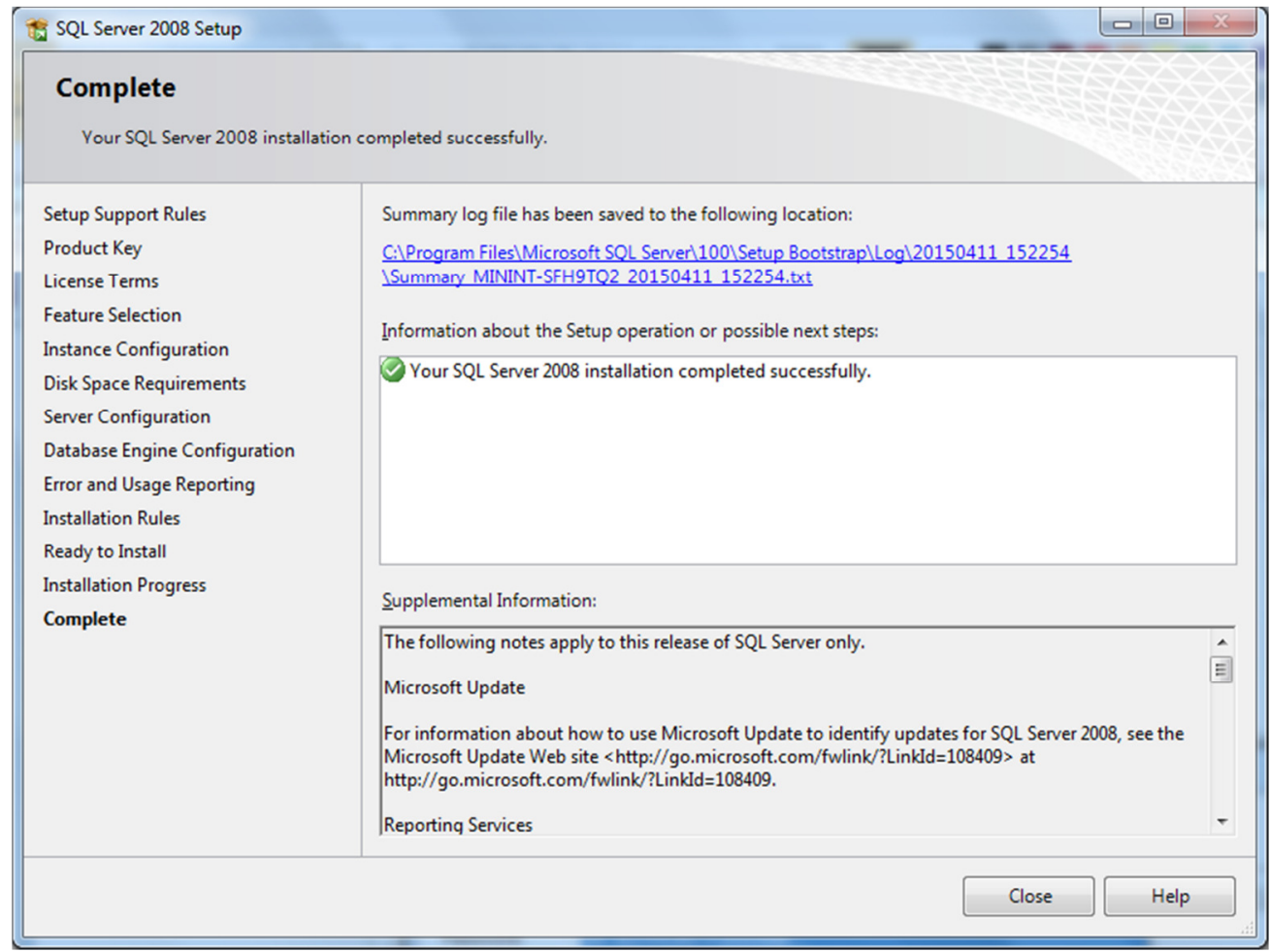

\section{c. Installation Procedure for Microsoft SQL Server 2008 SP4}

SP4 is an update to an existing SQL Server instance on a machine. Following are step by step instructions to update SQL server 2008.

- Click on SQLServer2008SP4-KB2979596-x64-ENU.exe to start set up.

- Wait for software to complete initial checks.

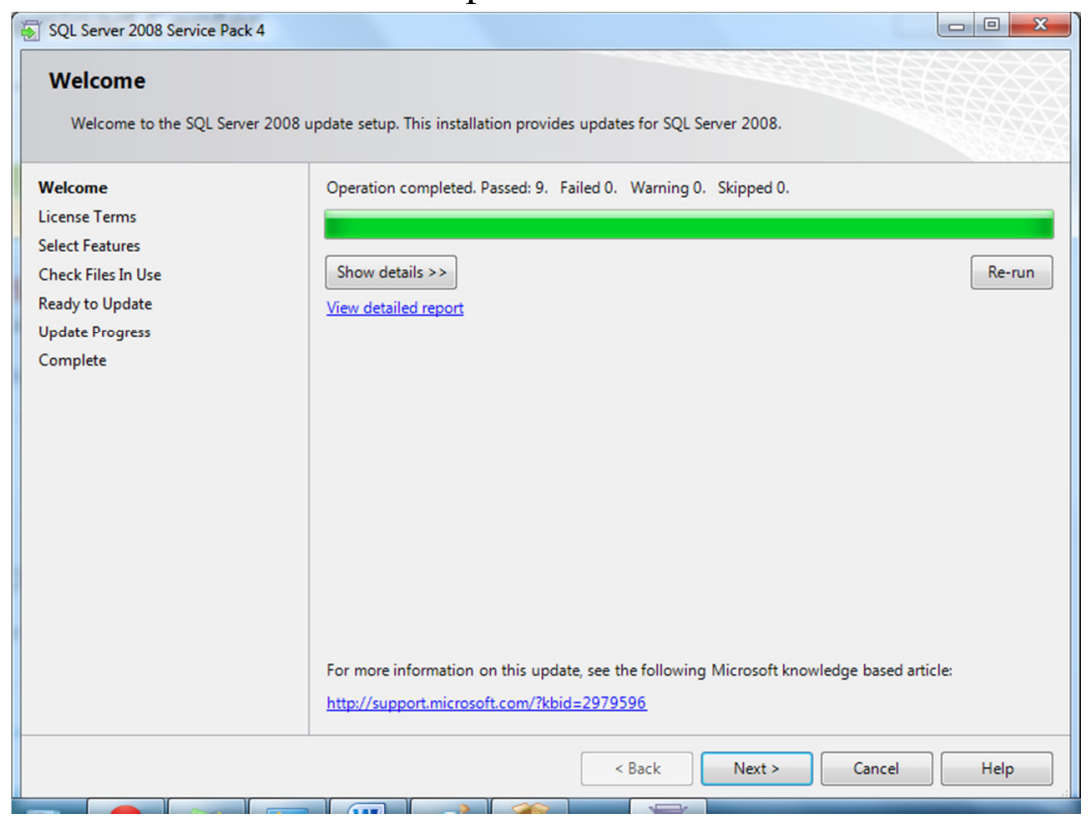


- Accept License terms. Then click 'Next'.

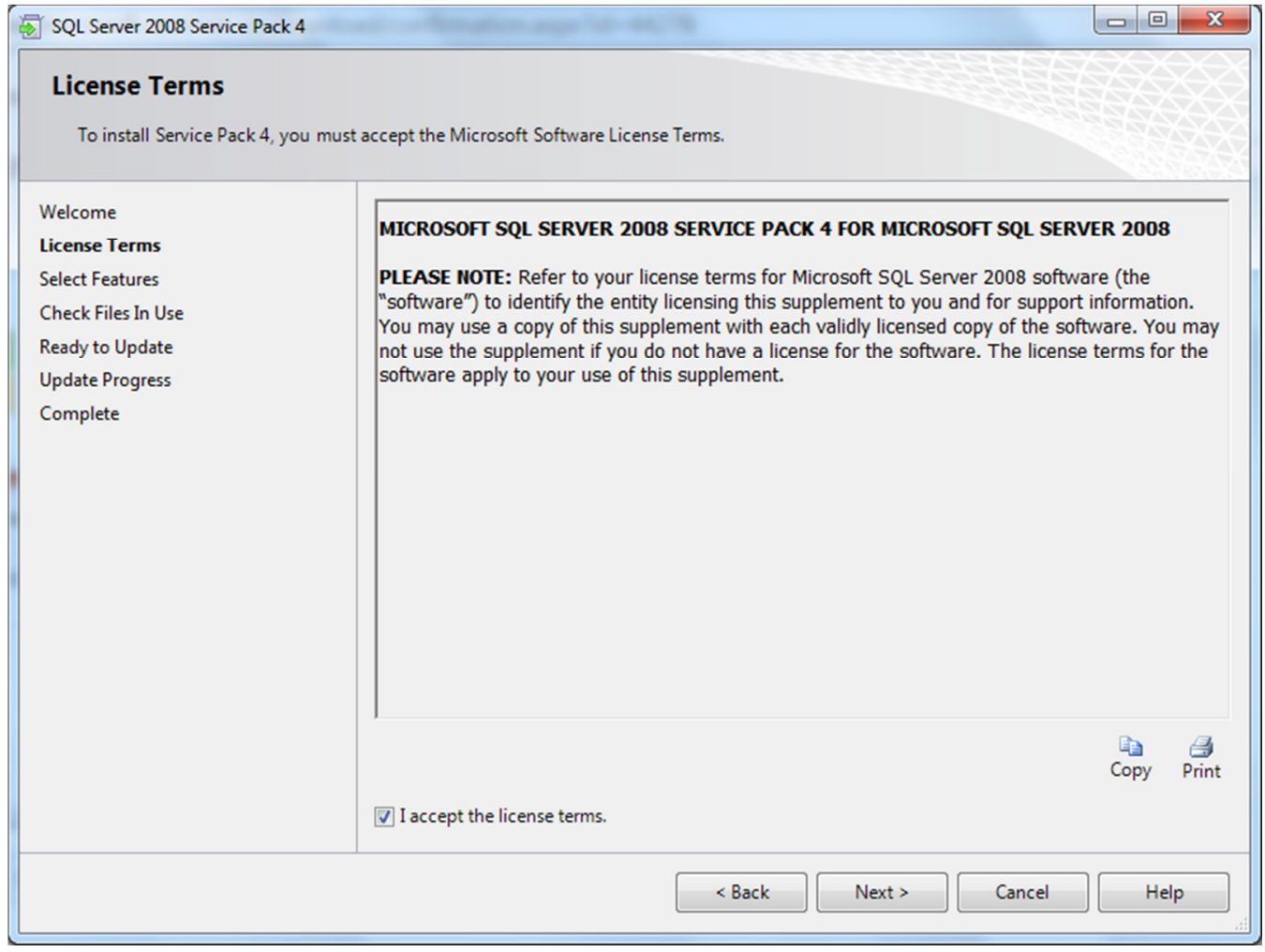

- Select all features to update.

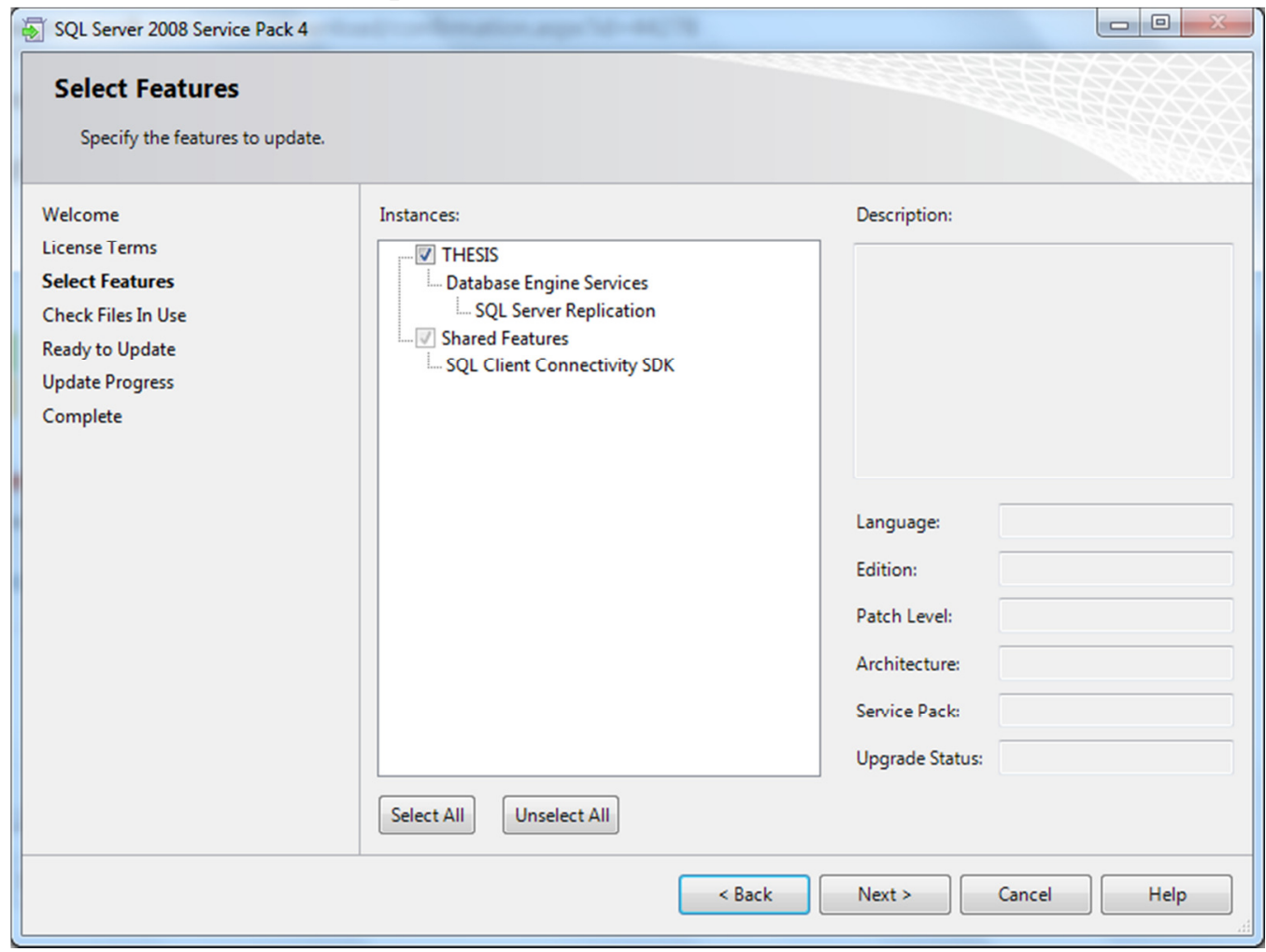


- Check files in use that may interrupt installation.

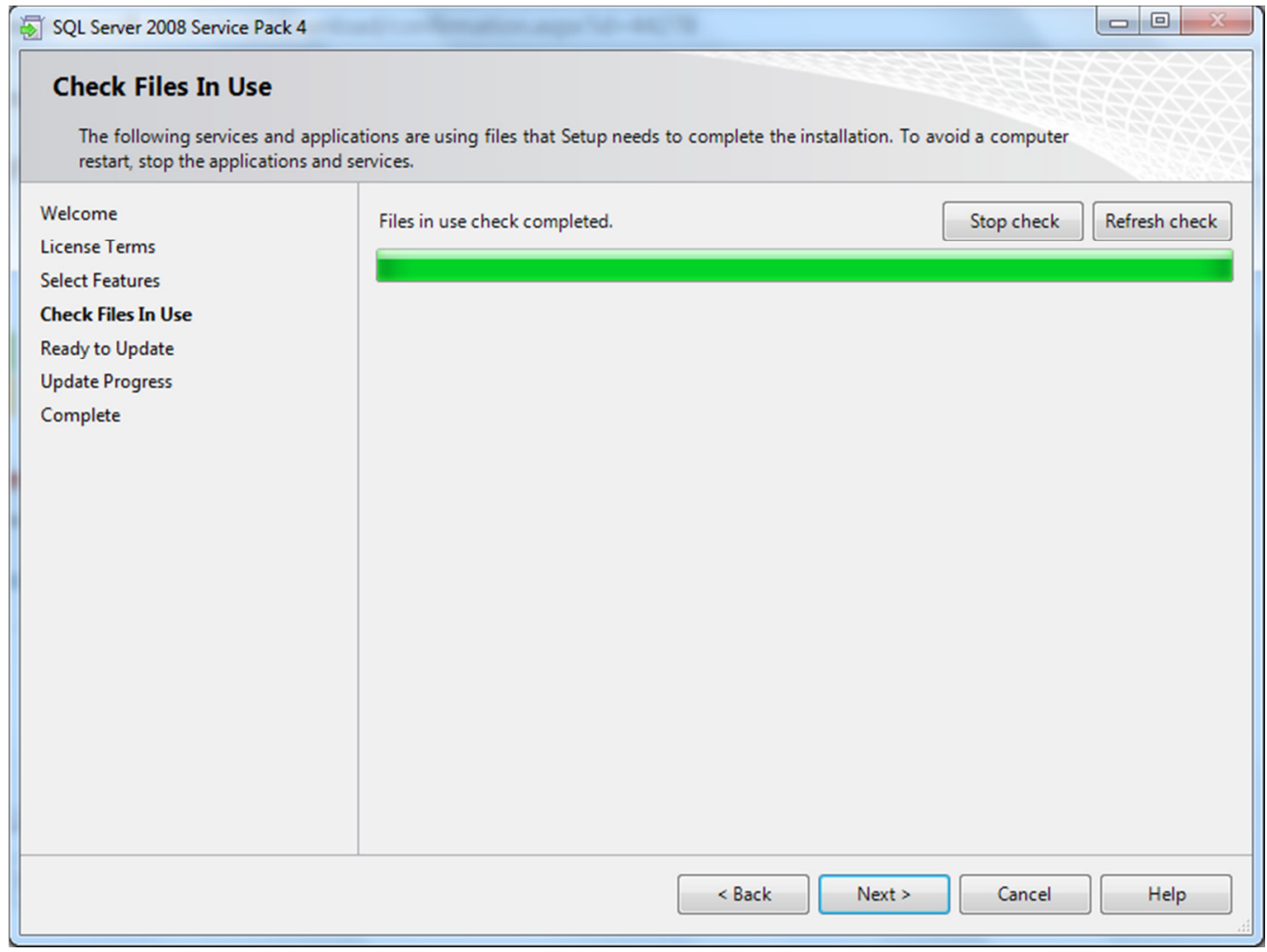

- Click Update

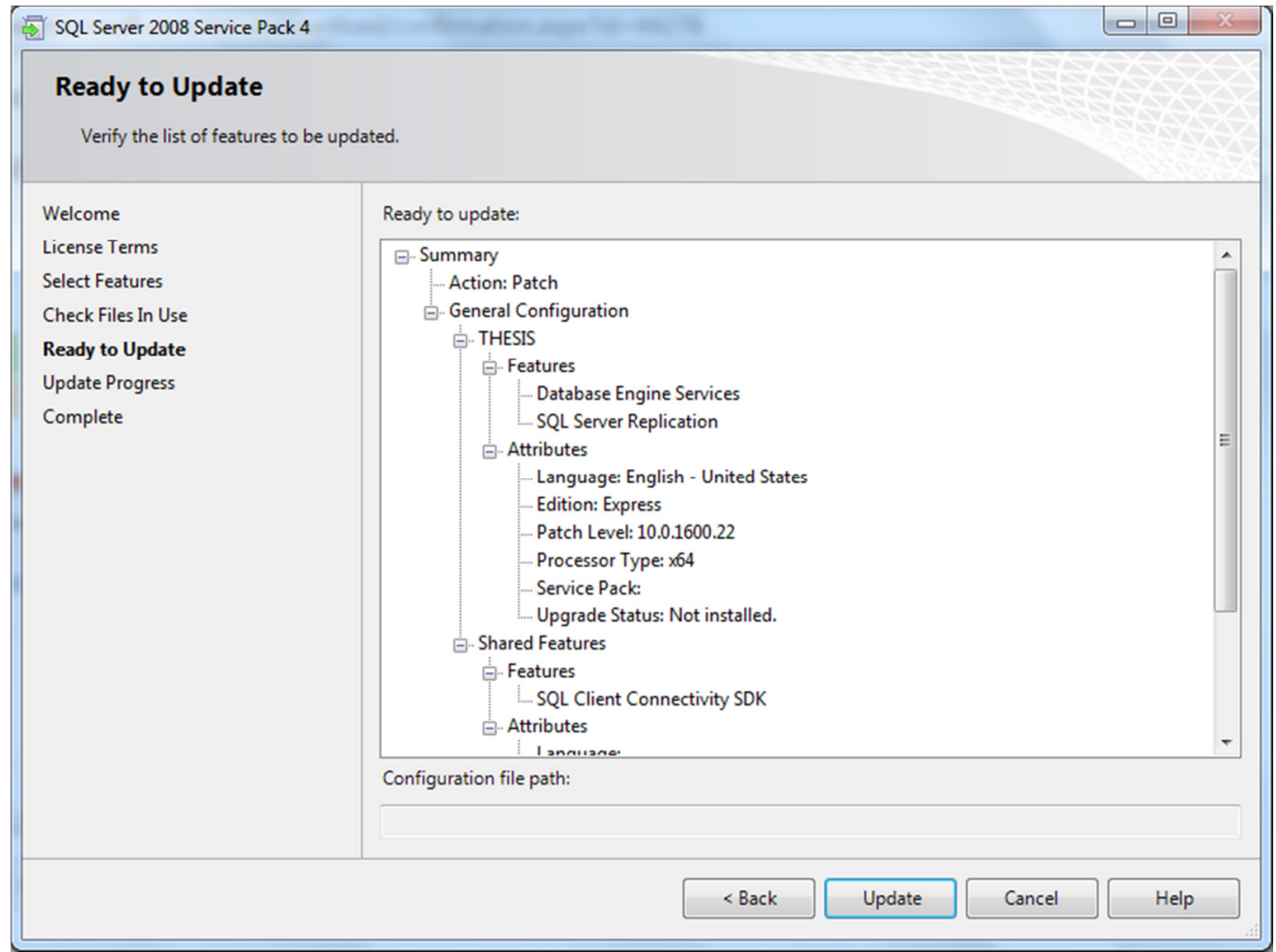


-Wait for the update process to complete and then check for completion message.
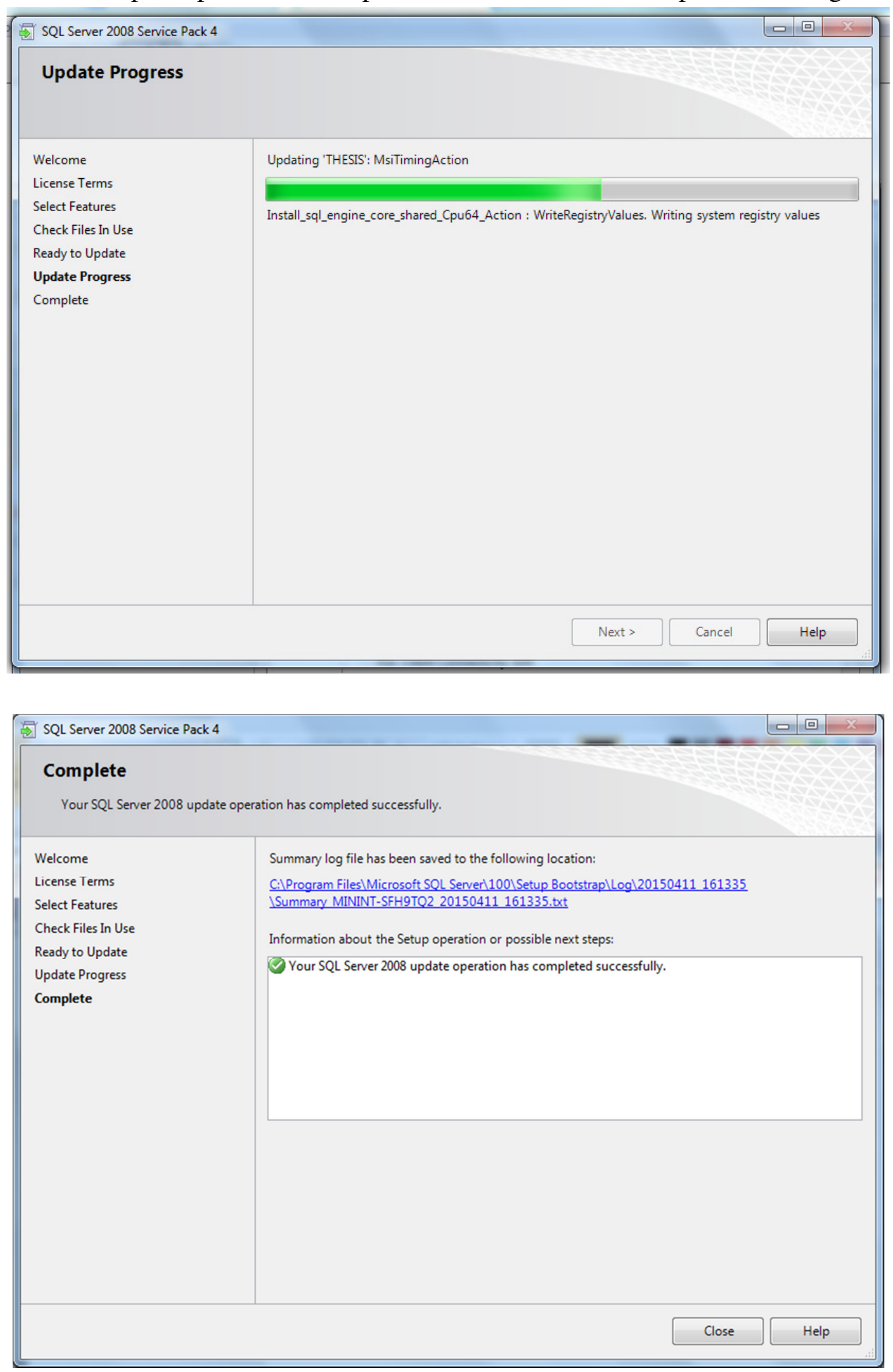


\section{d. Installation Procedure for Microsoft SQL Management Studio 2008}

Follow step by step instructions to install SQL Management studio.

- Click on SQLManagementStudio_x64_ENU.exe to start installation.

- If Compatibility message pops up, if SP4 is already installed as per instructions click on 'Run program' else click on 'Cancel' and install SQL Server 2008 SP4 to install management studio.

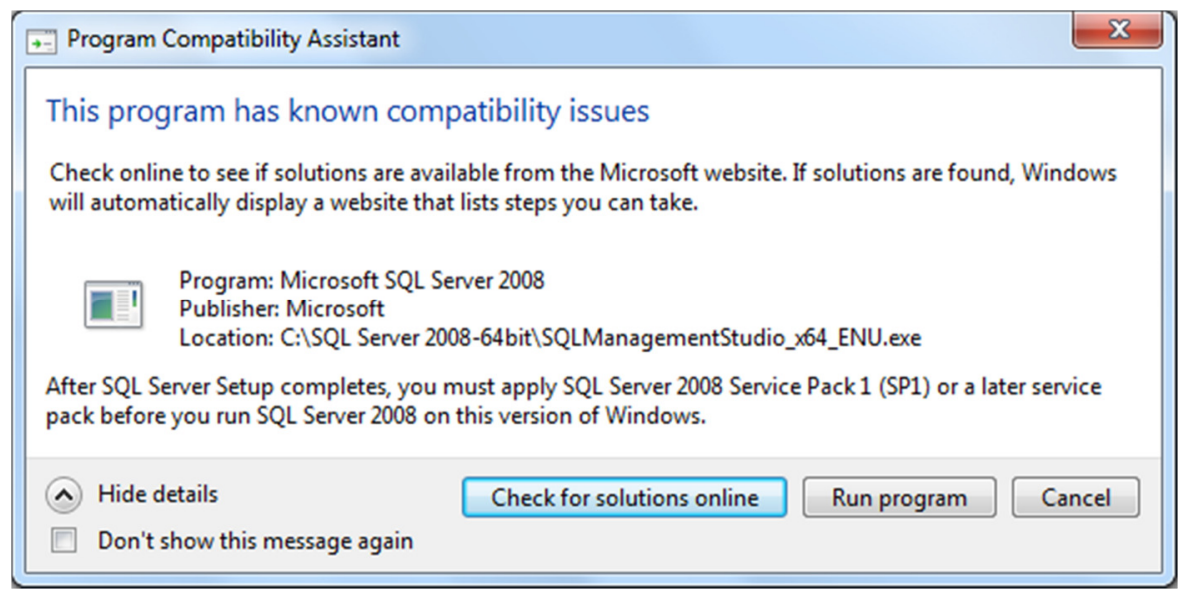

- Check for Setup Support requirements and then click on install Setup Support files.

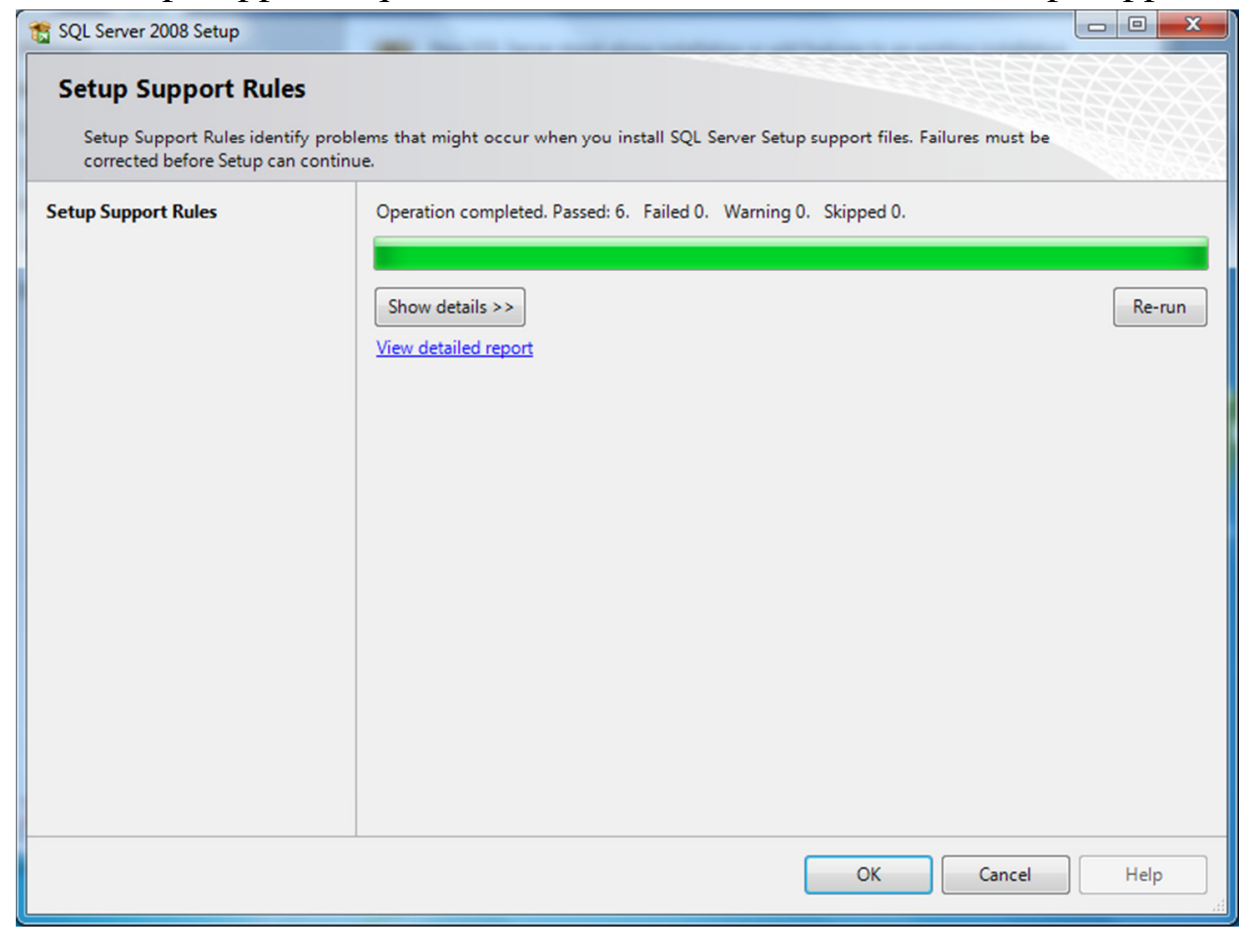




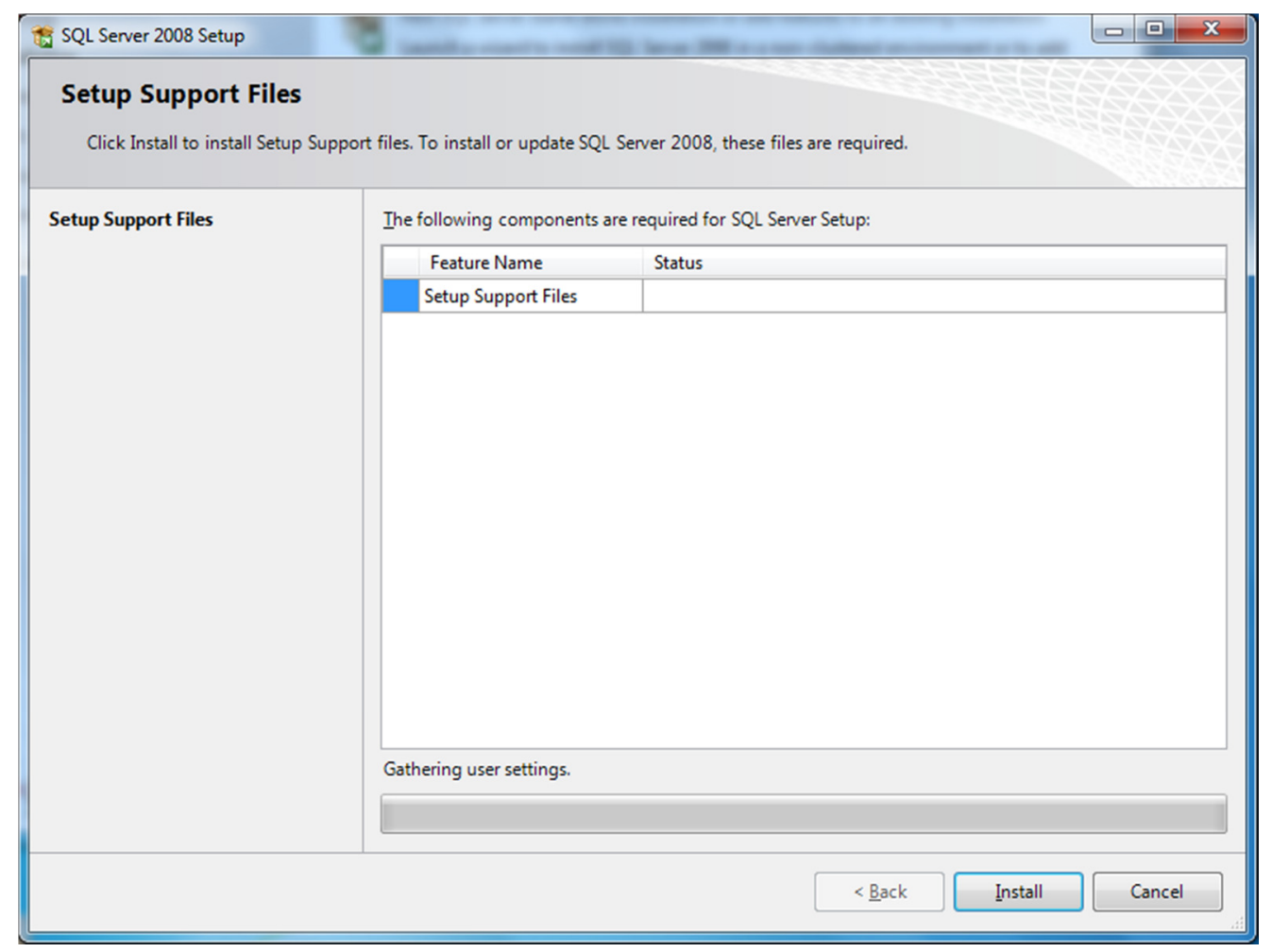

- Wait for installation to complete and then click 'Next'.

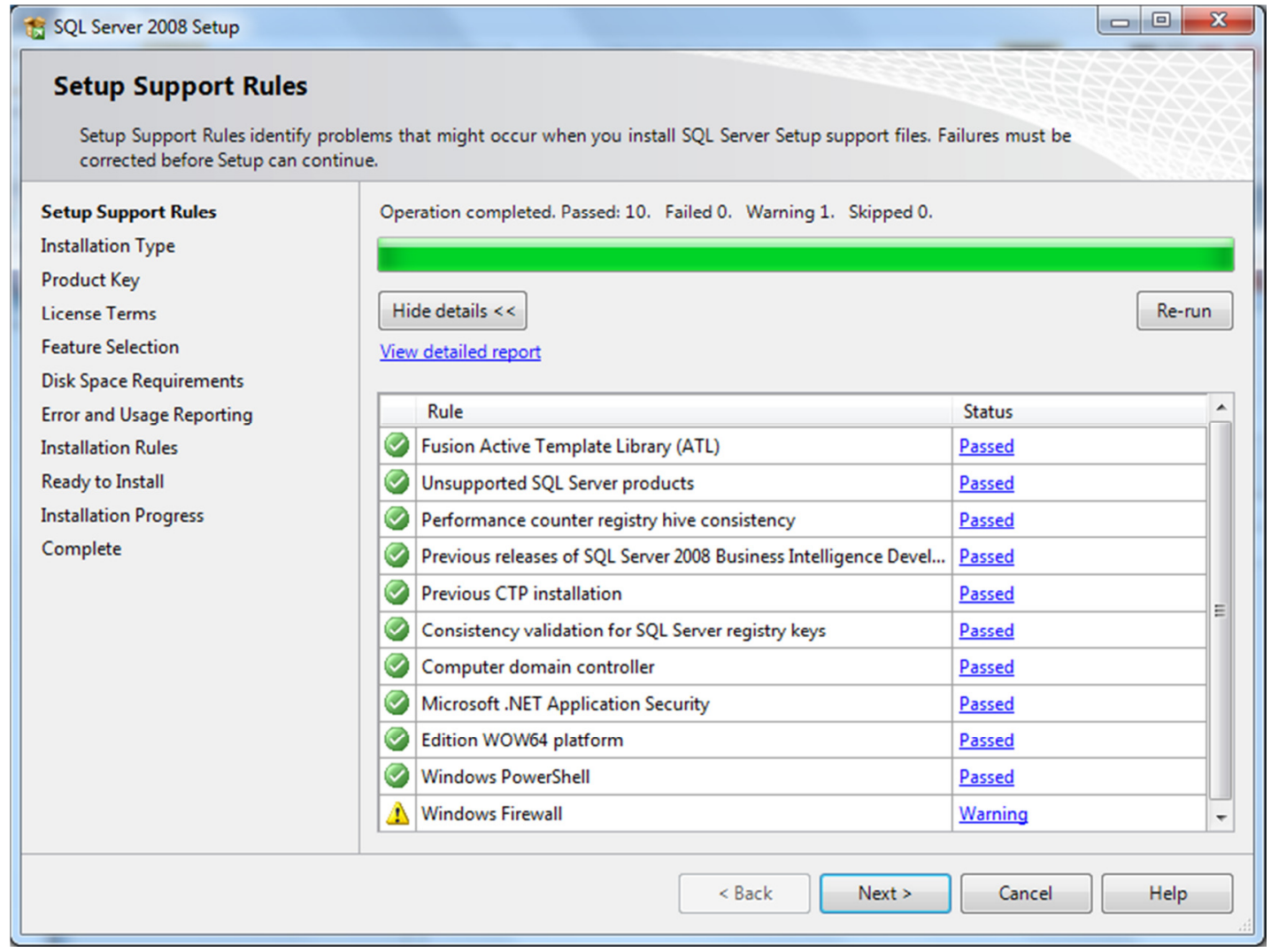


- In Installation Type, select new installation.

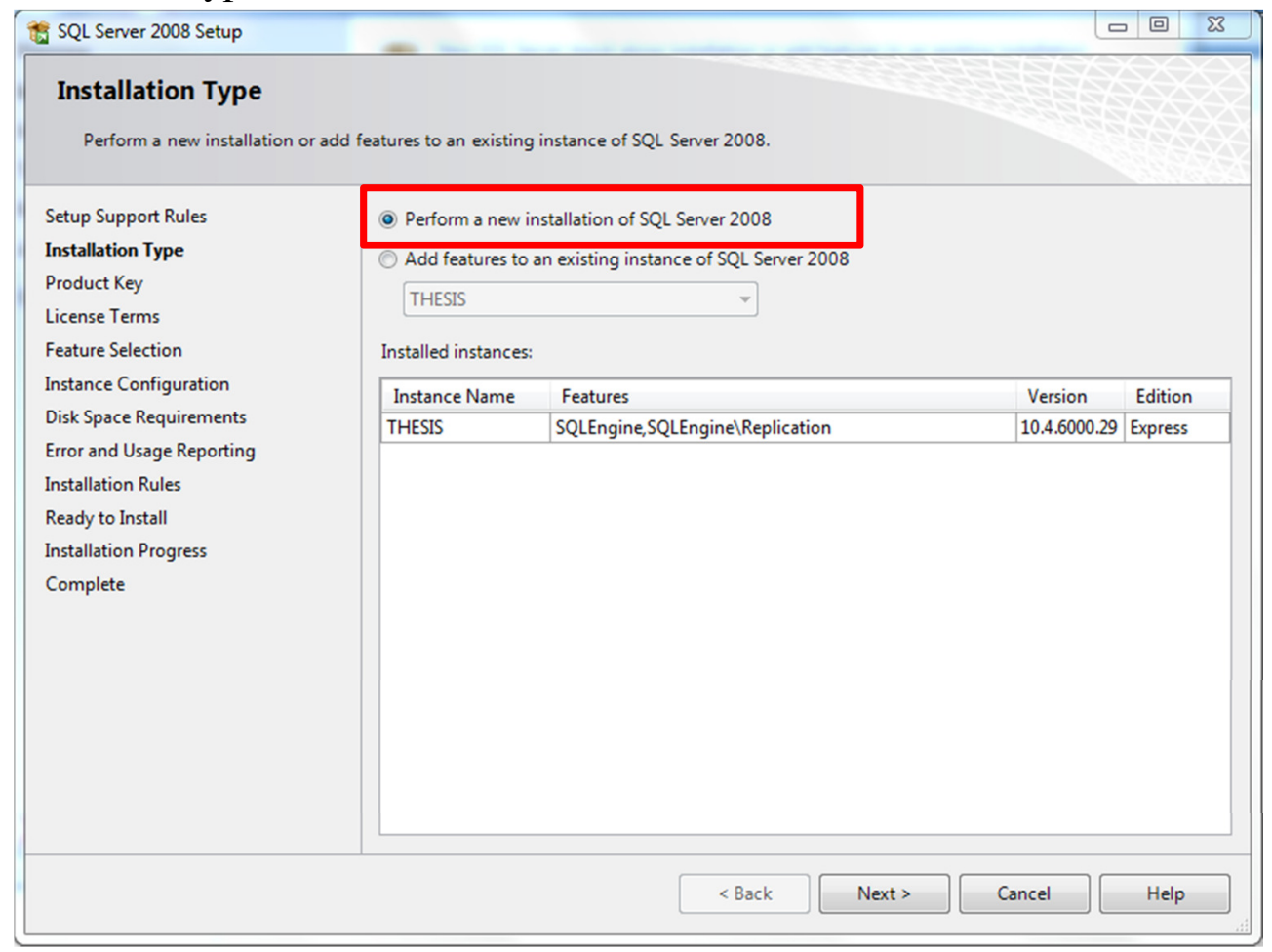

- Since software used is express edition, which is free, product key is not required.

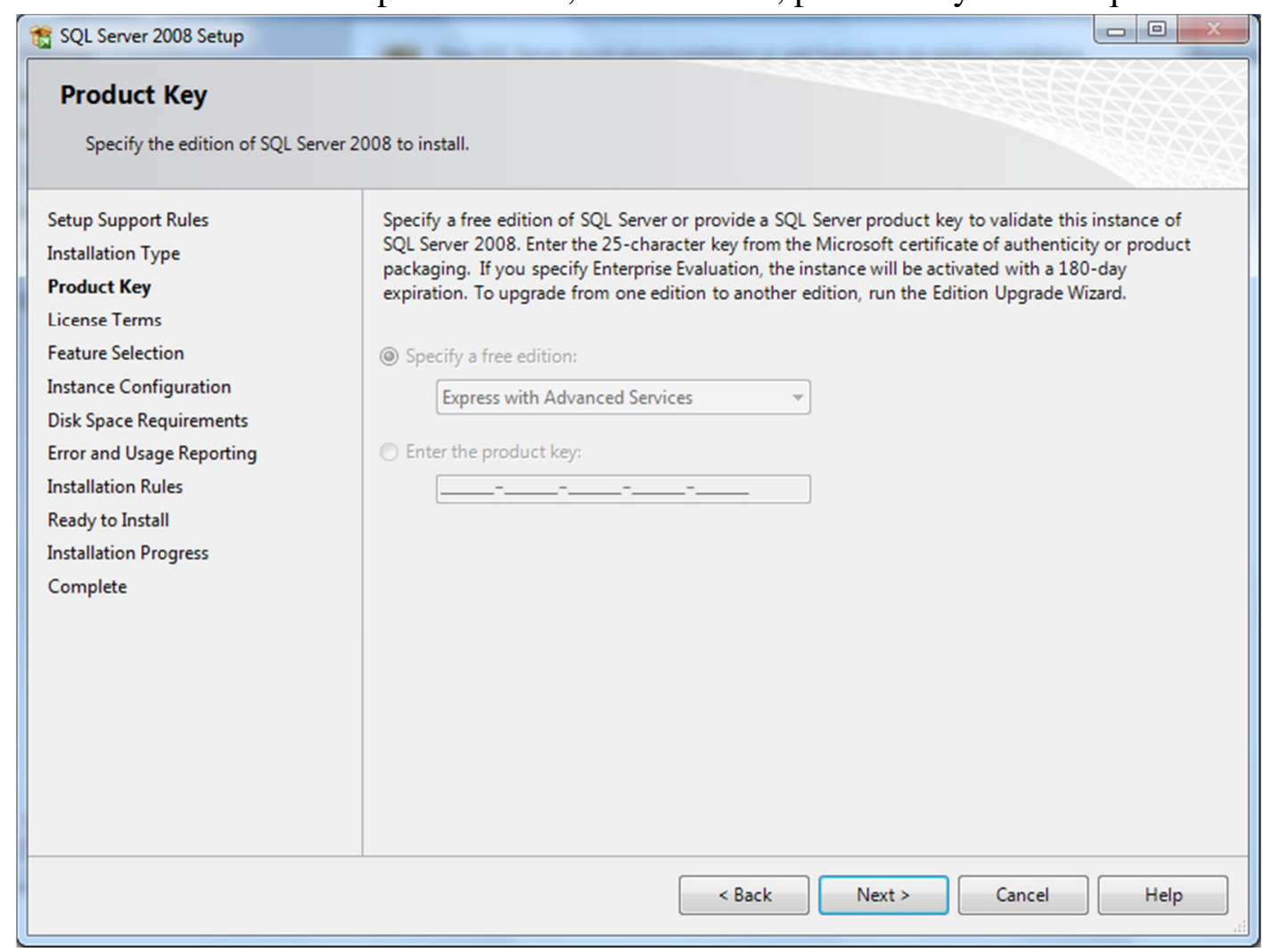




\section{- Accept License terms.}

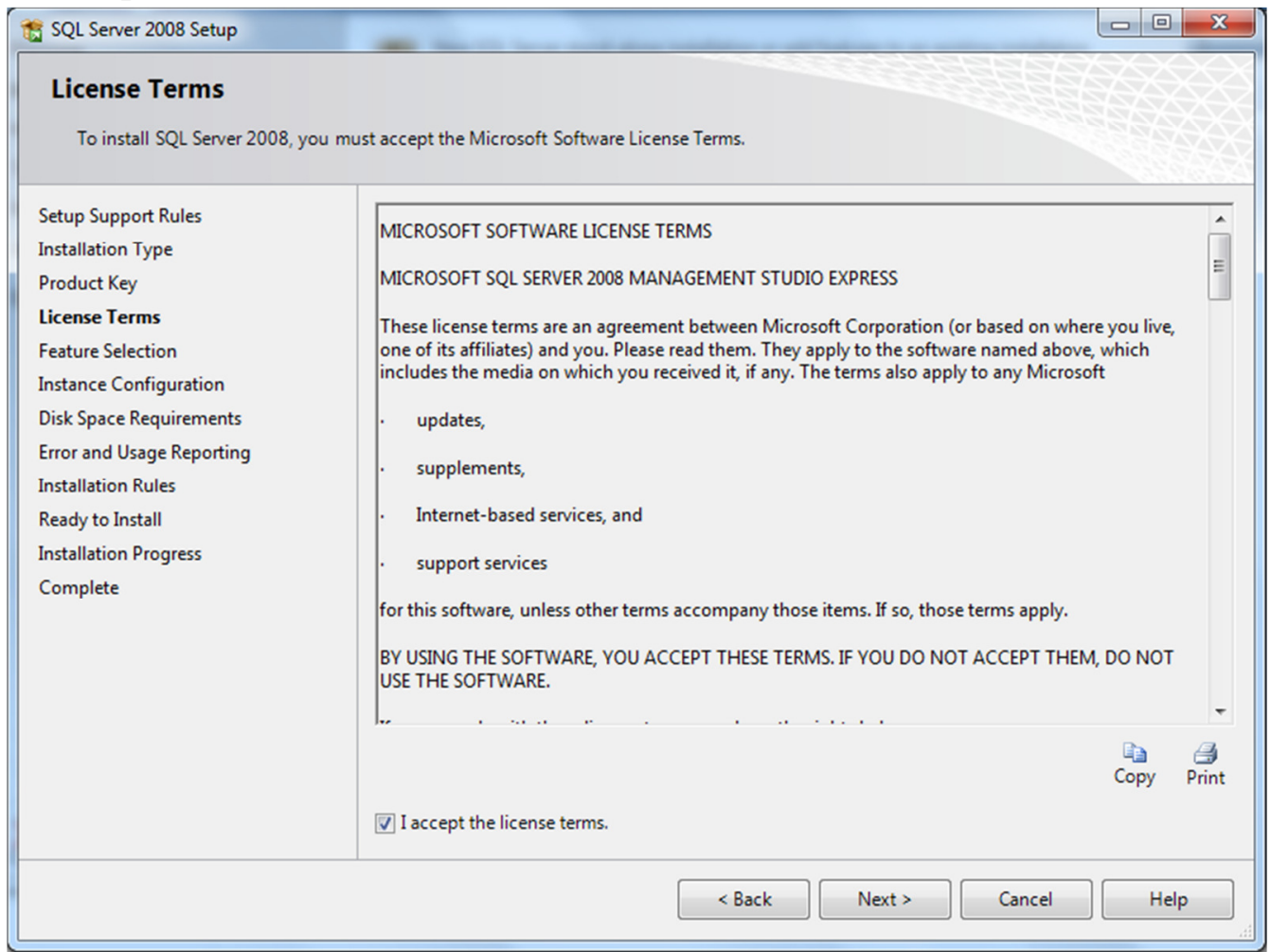

\section{- In feature selection select 'Management Tools'}

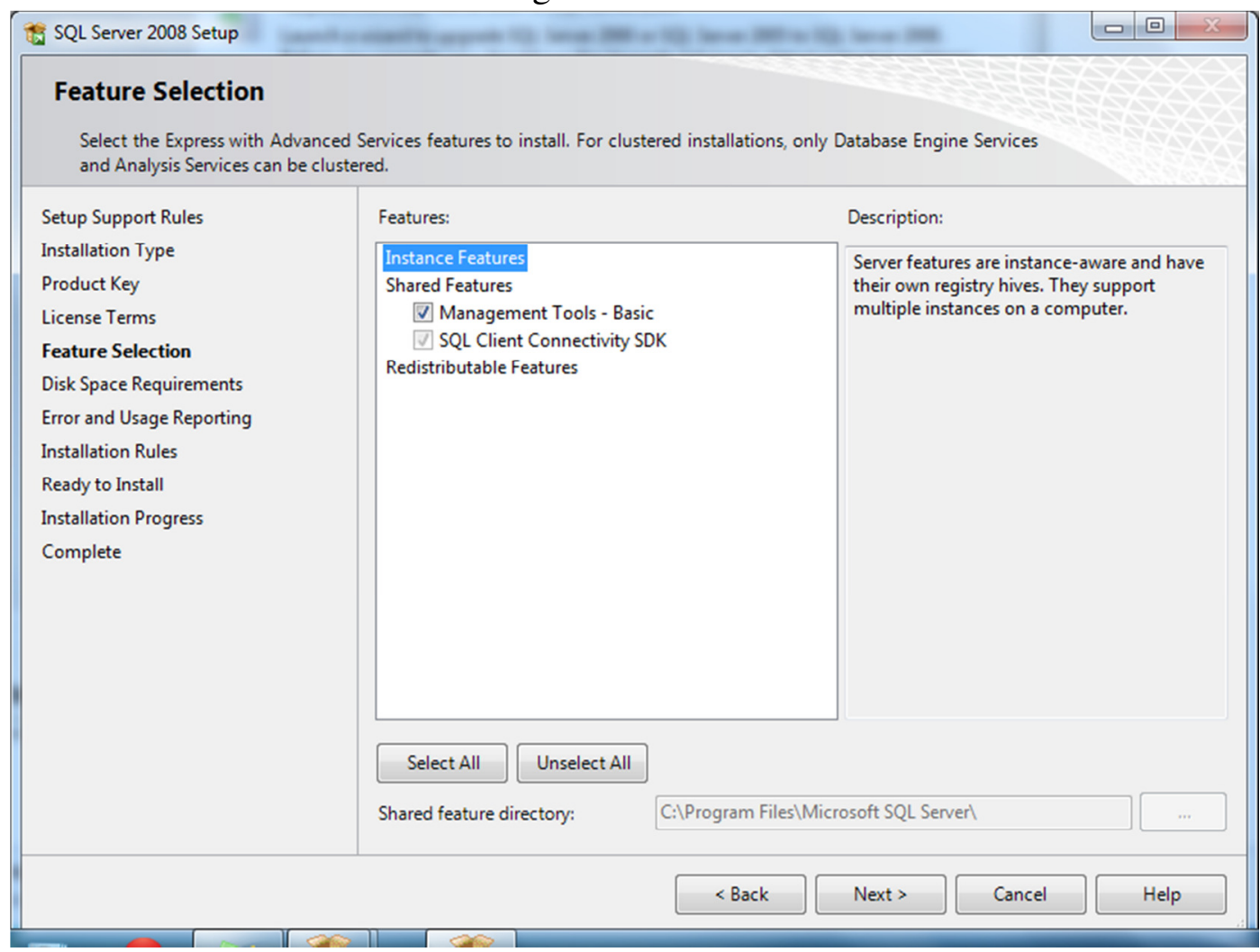


- Check for disk space requirements.

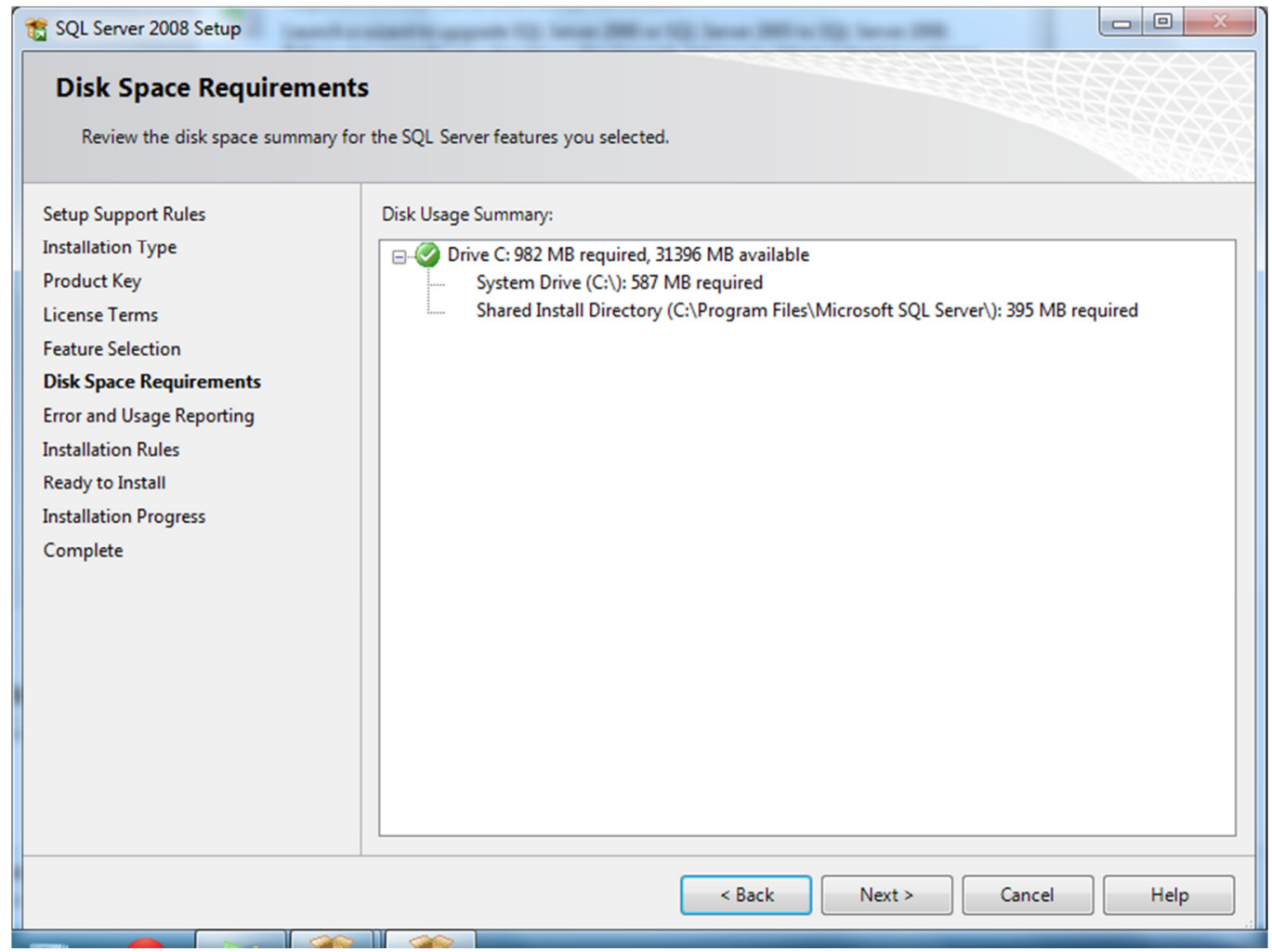

\section{- Select error reporting preferences.}

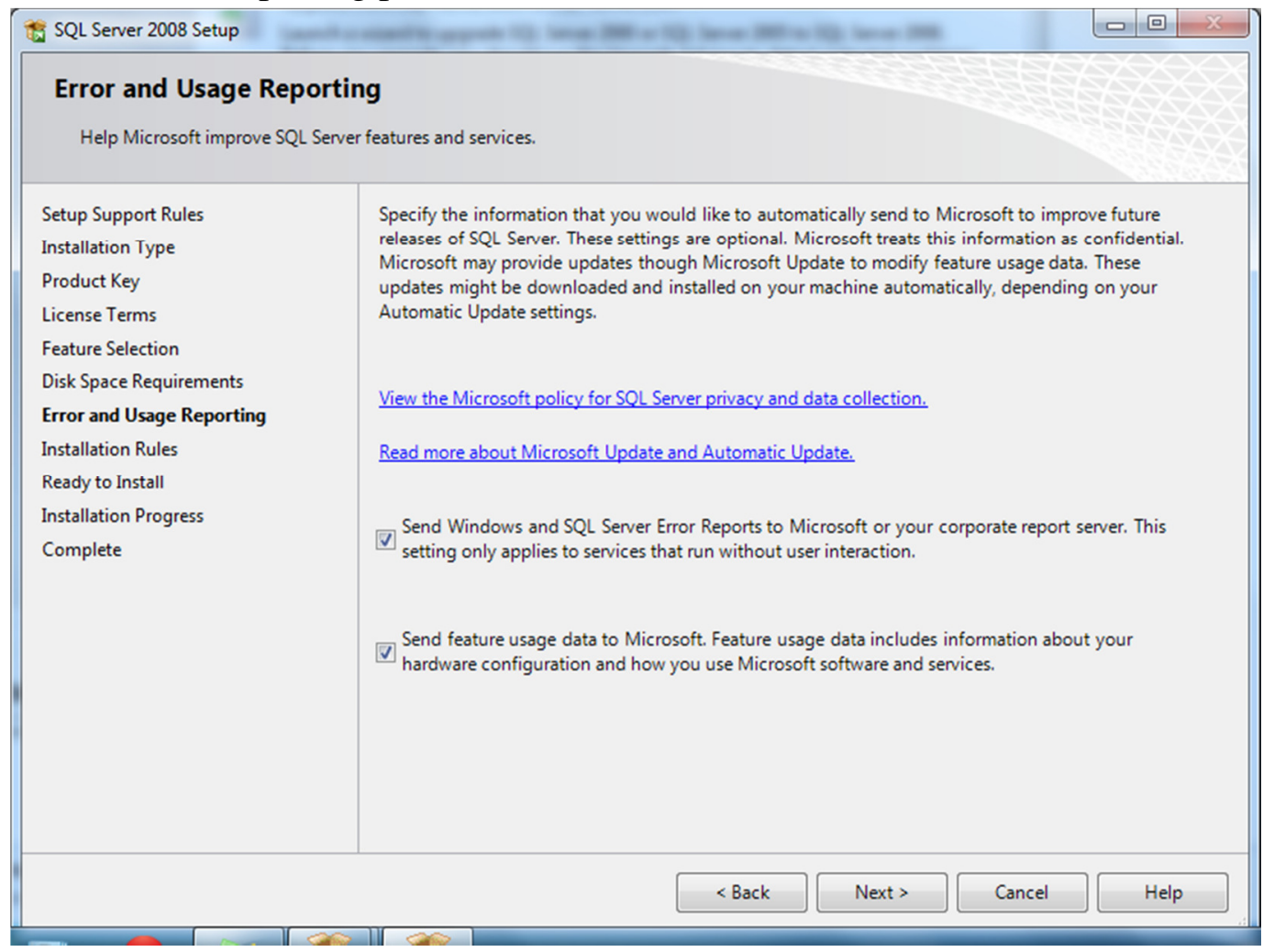


- Check for installation rules.

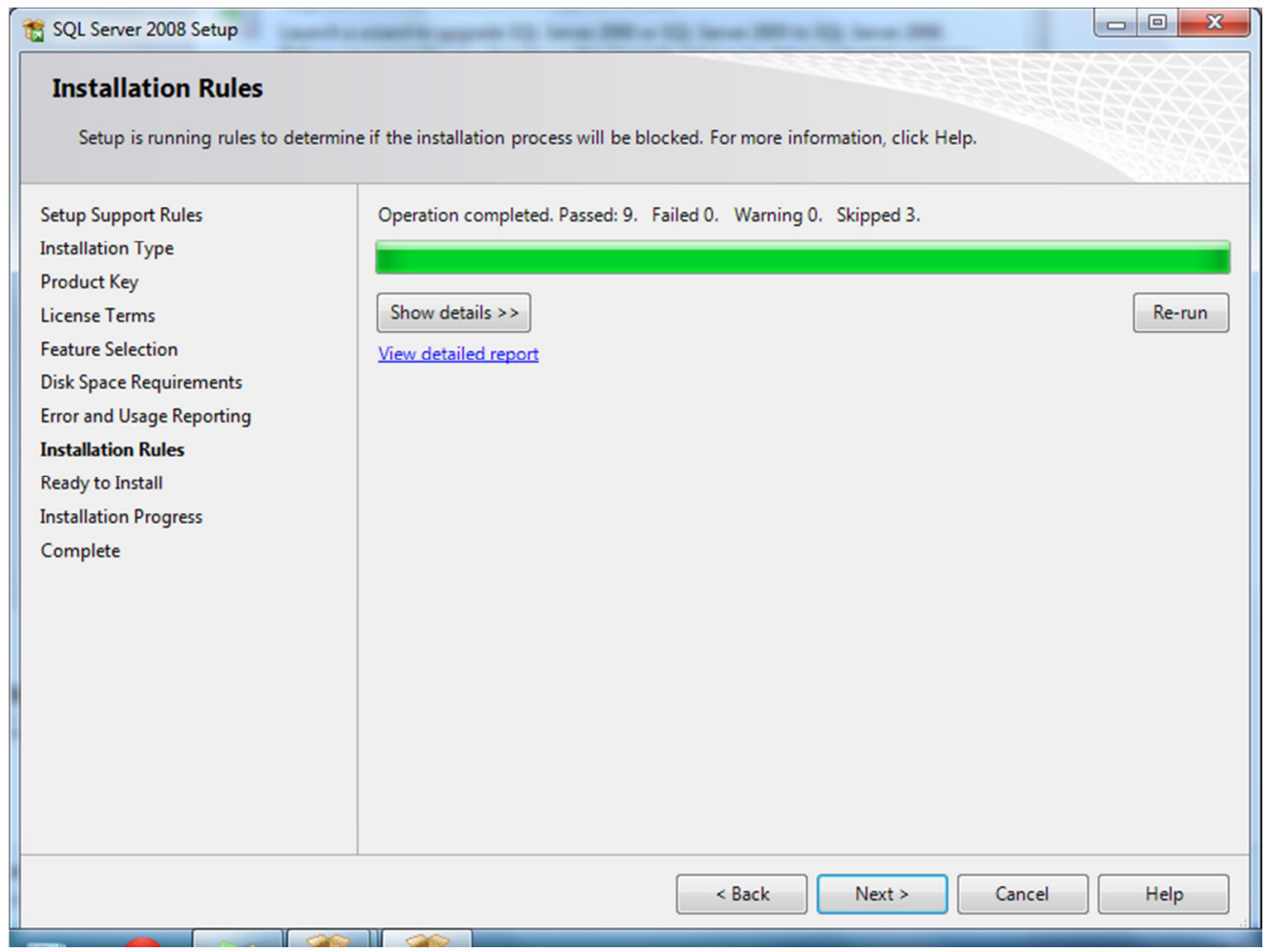

- Click on 'Install'.

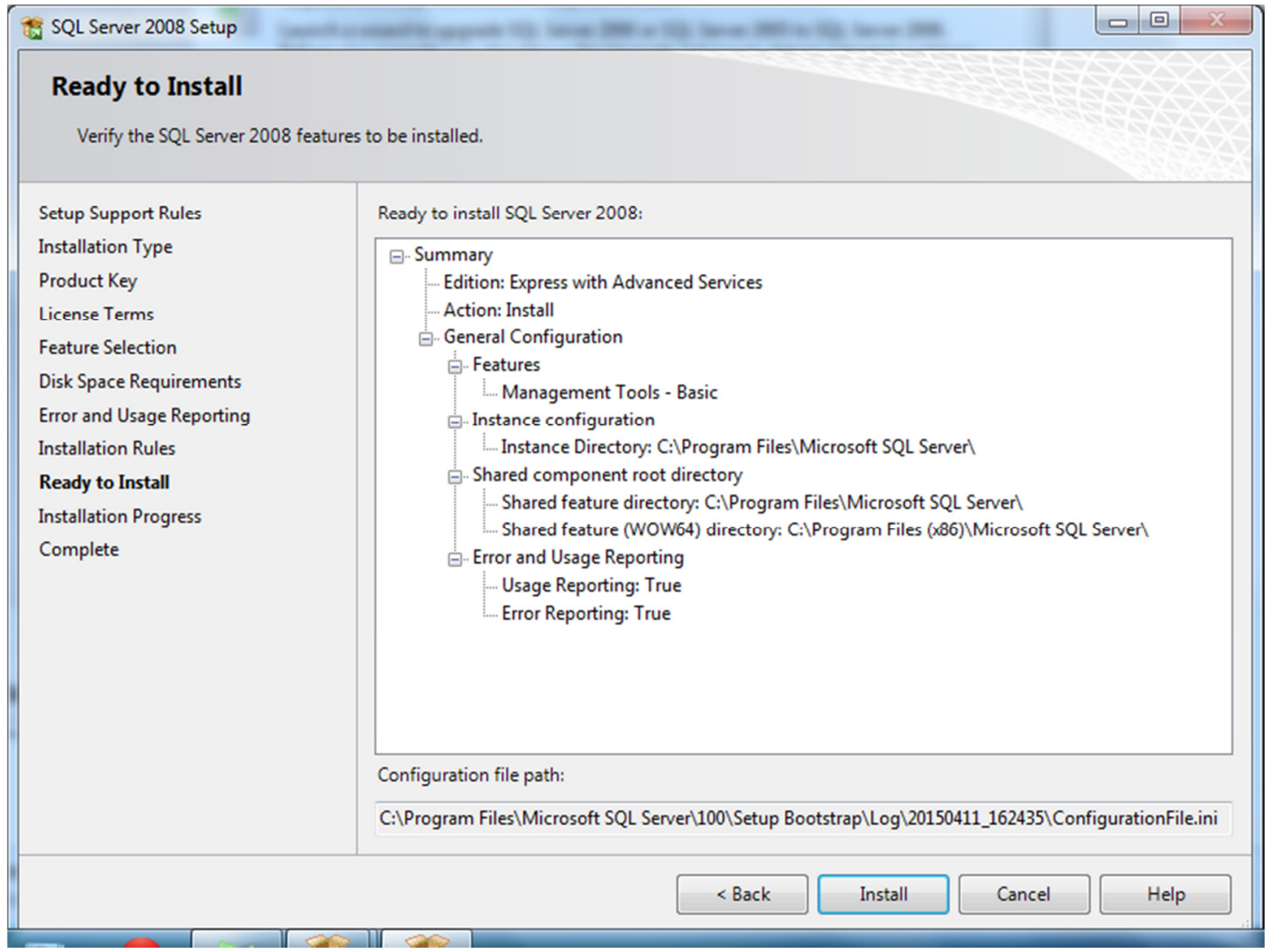


- Wait for installation to complete and then click 'Next'.

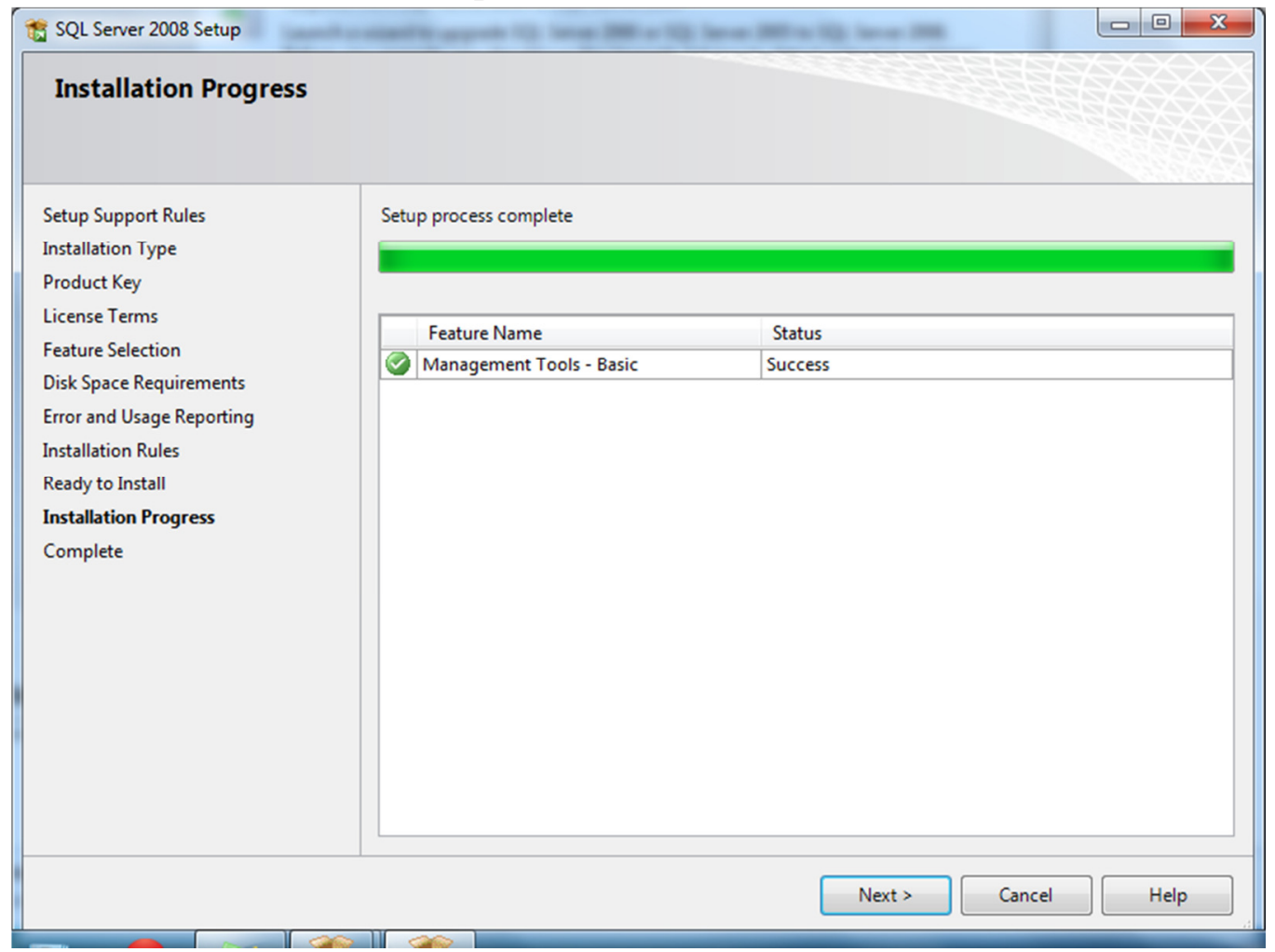

- Check for the completion message and then close the installation.

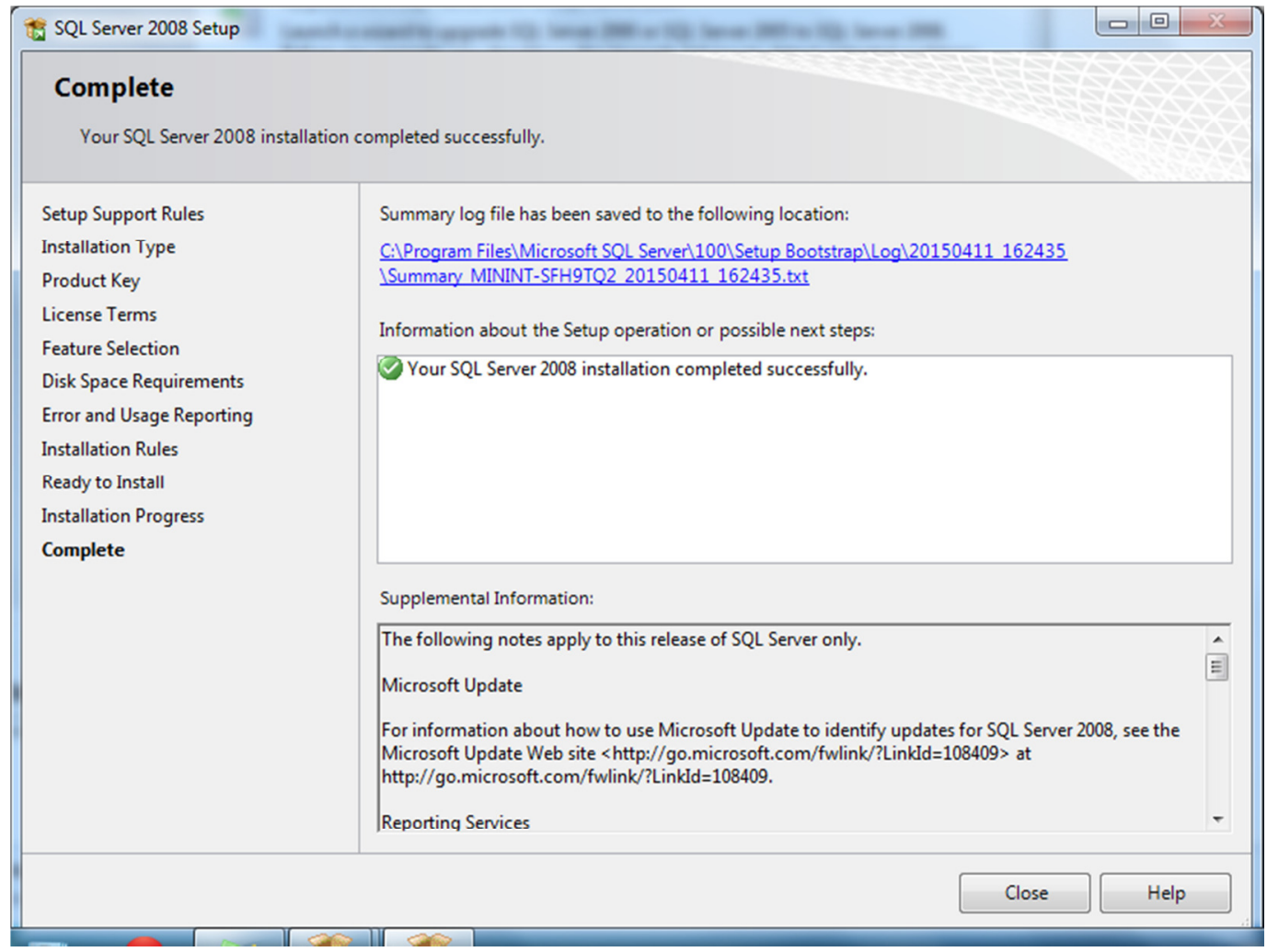


e. Loading database.

- To open SQL Management Studio, go to start menu. In 'Microsoft SQL Server 2008' folder, click on 'SQL Server Management Studio' file.

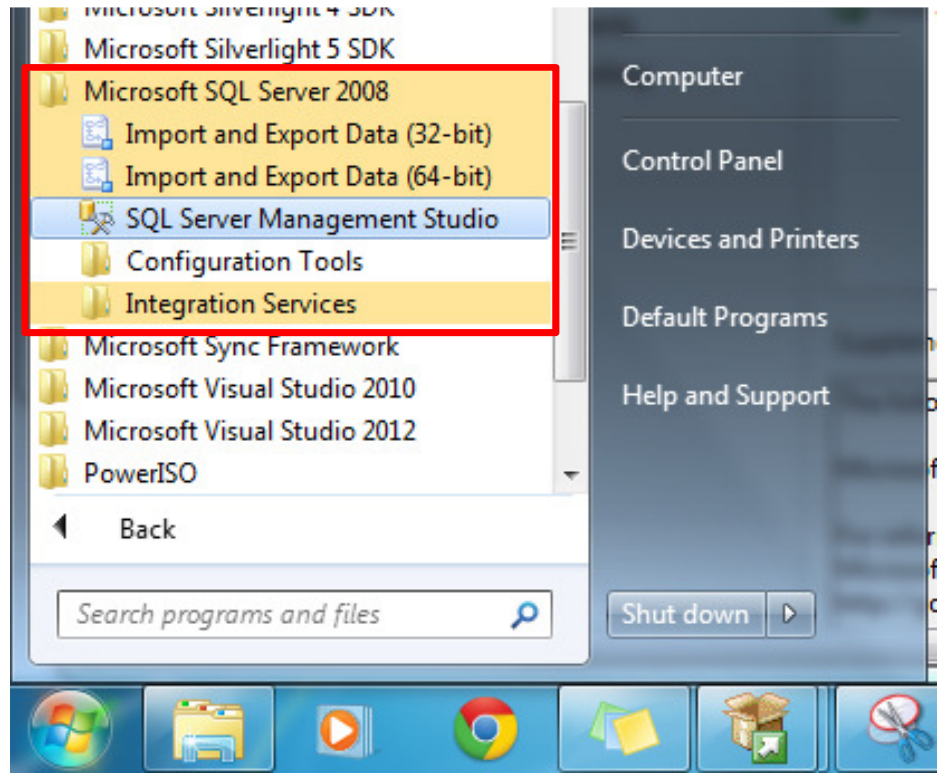

- In Management studio, select database to work.

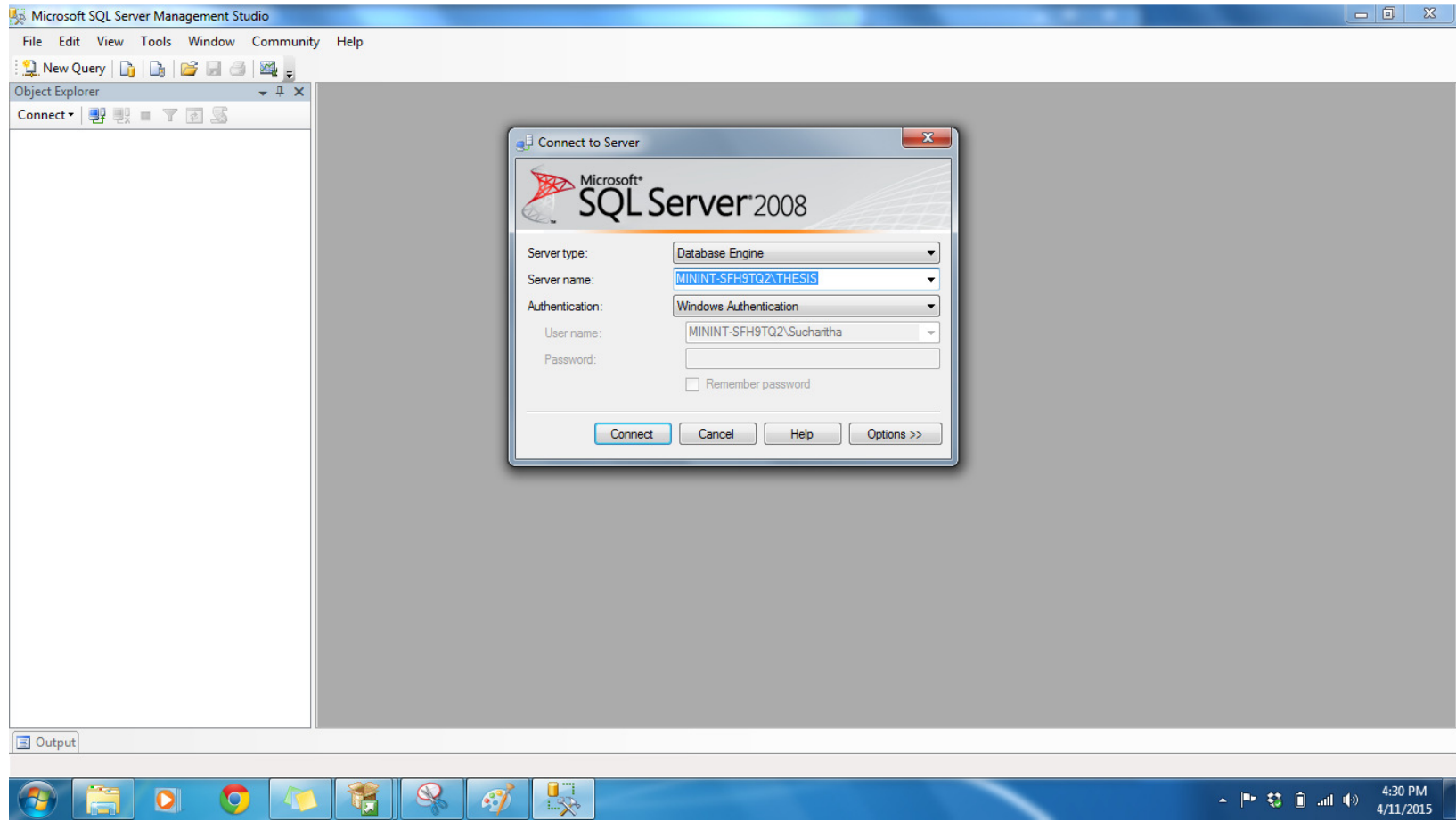




\section{f. Creating Database Entities}

- Connect to 'THESIS' database in SQL server Management Studio.

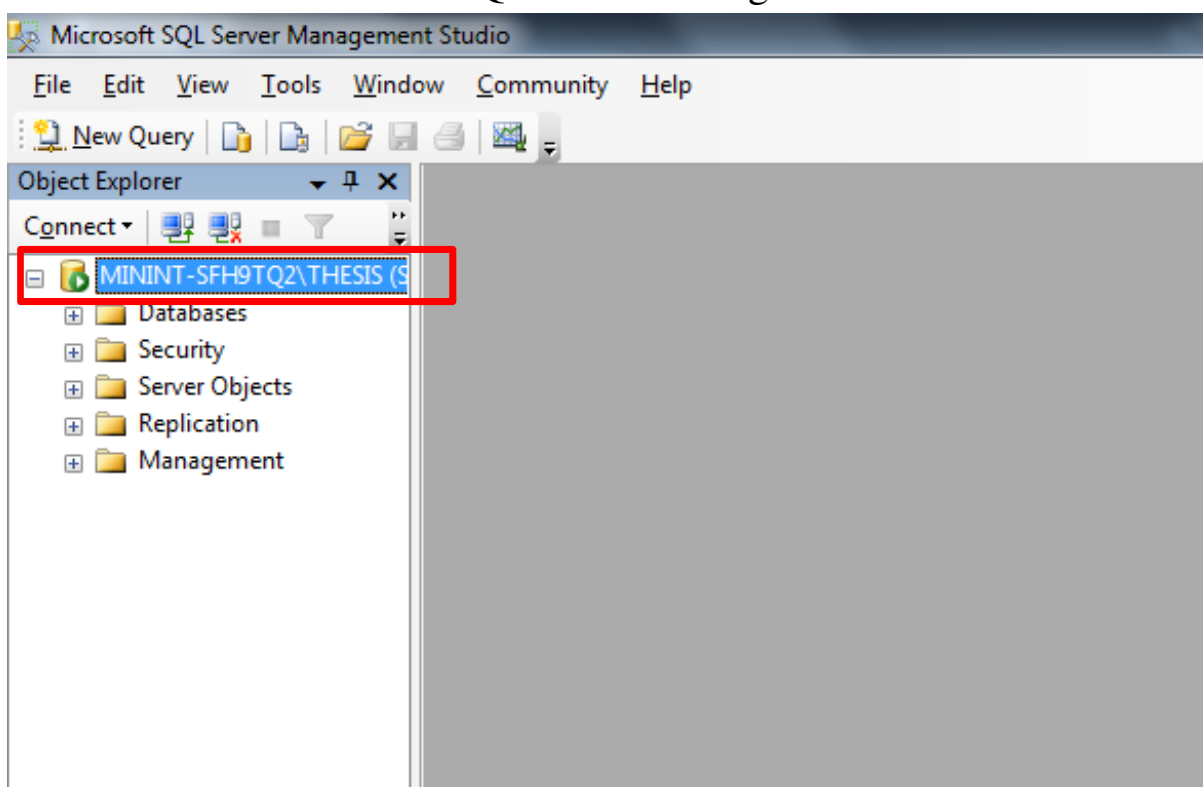

- In the instructions new database is created named 'Chapter13'

- Copy the scripts from Appendix A to create tables and procedures. In this picture, script to create table 'RCMemberConfiguration' is copied to a sql file. Click on 'Execute' to run the script. Similarly create all the tables.

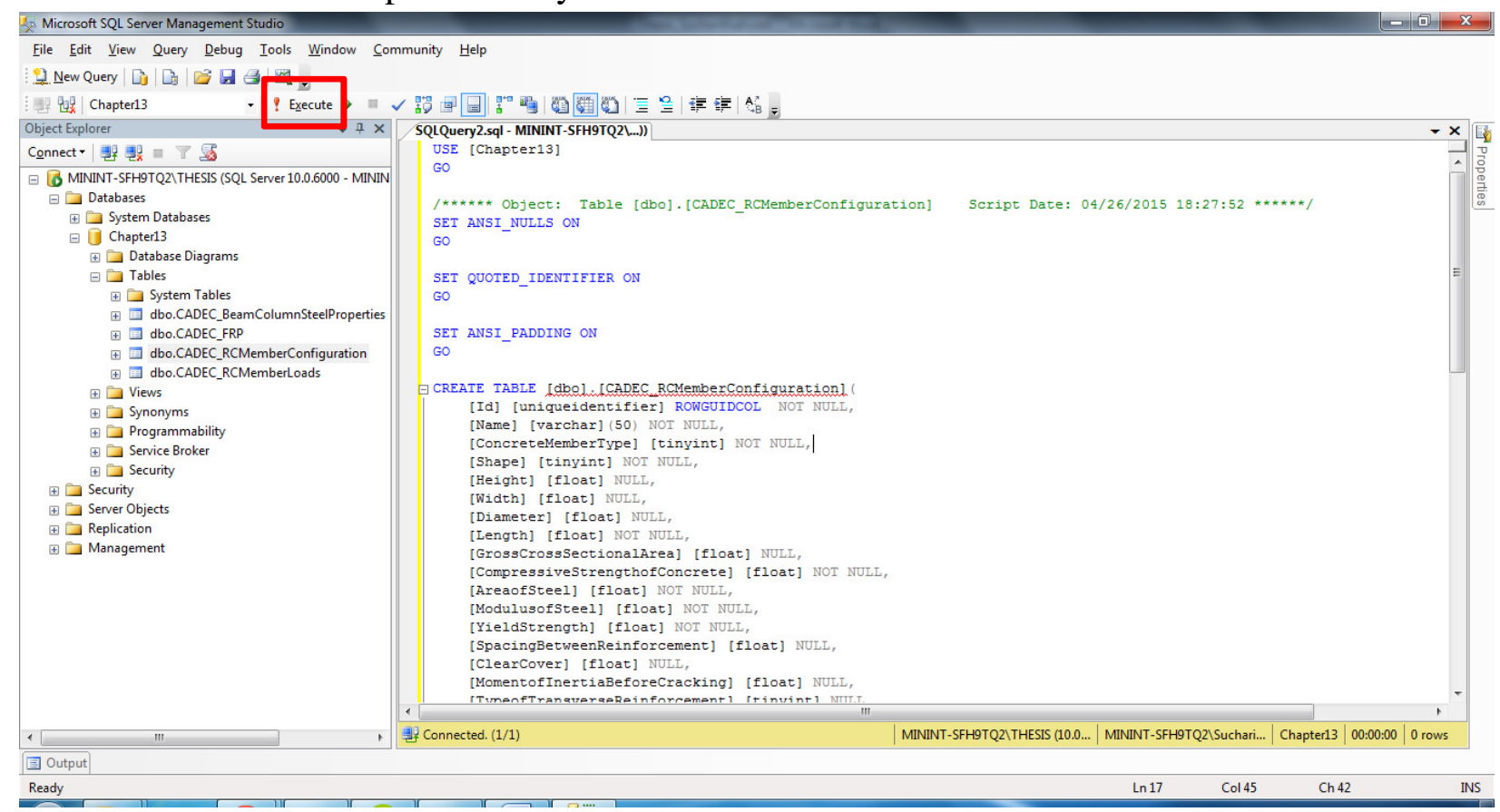


- In the following picture, script to create procedure 'CADEC_RCMemberConfiguration_All' is copied to a sql file. Click on 'Execute' to run the script. Similarly create procedures for all the tables. Procedures can be viewed under Chapter13>Programmability $>$ Stored Procedures

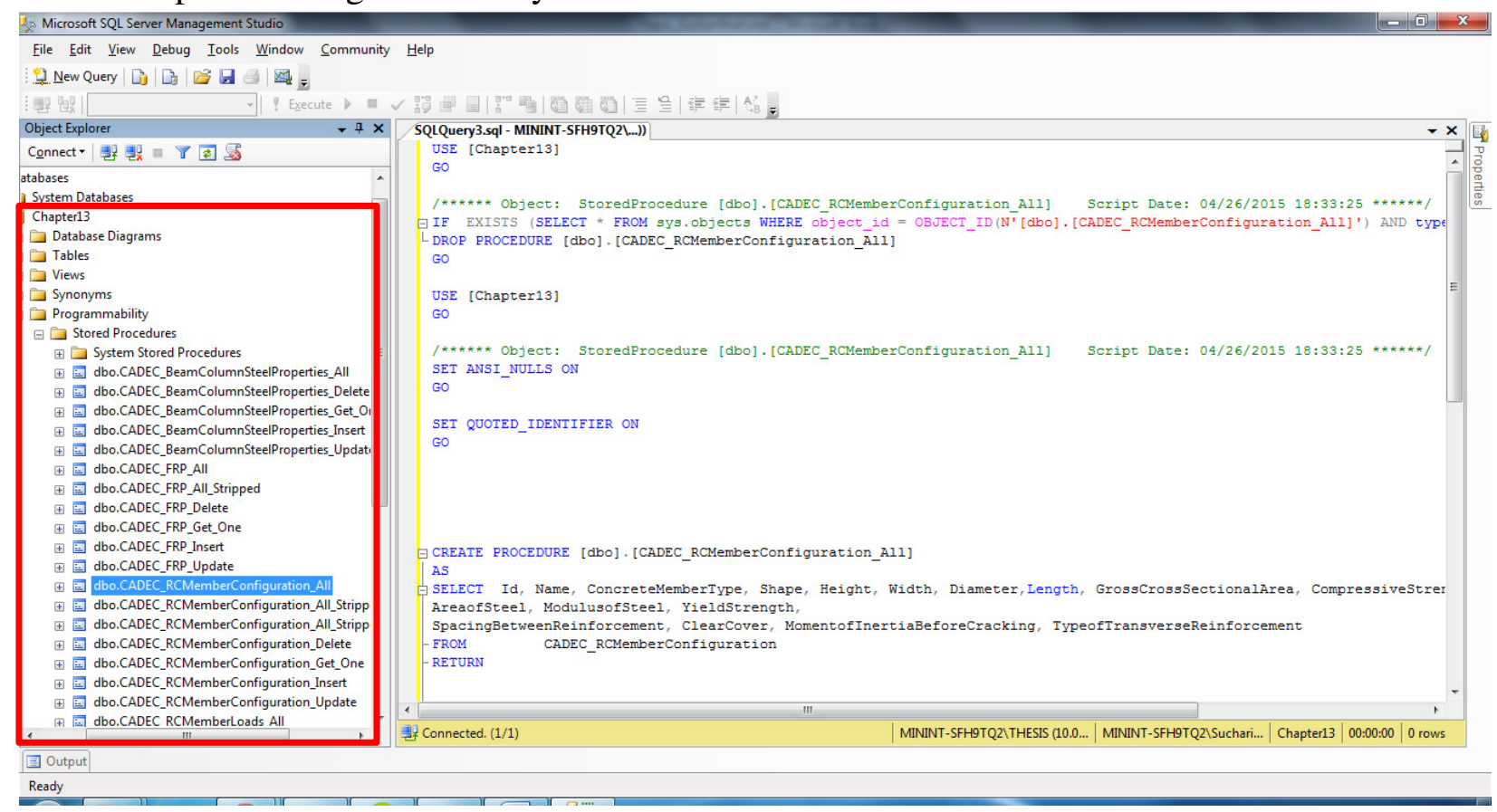

g. Configure Database in Visual Studio and store RC Member Properties.

- To open Web application in Visual Studio 2010, open the file 'ReinforcedConcrete.sln' from project folder, here 'Thesis'.

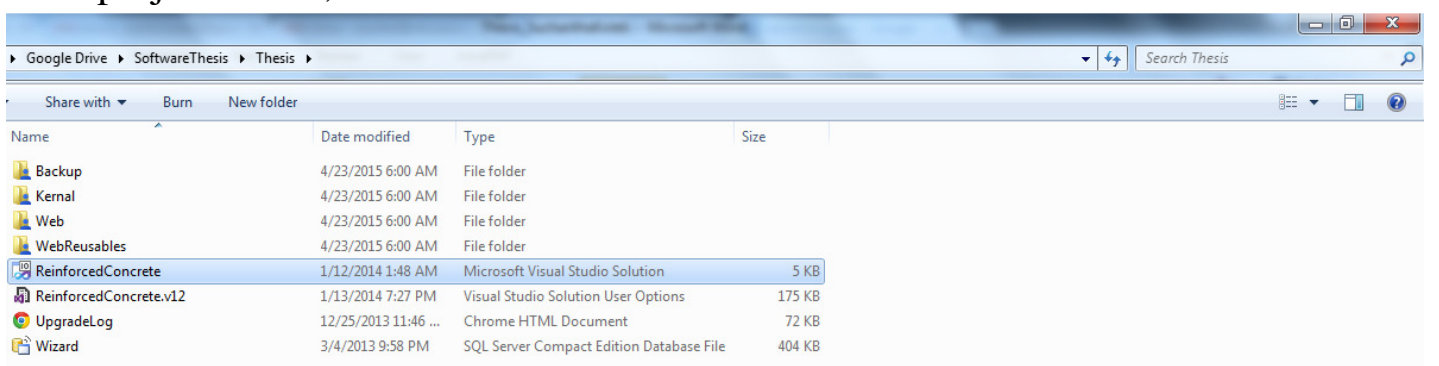


- To connect Web App to database, configure database in web.config file. In connection string, data source is database instance name, Catalog is database name and integrated security is true if windows authentication is used to login to the database.

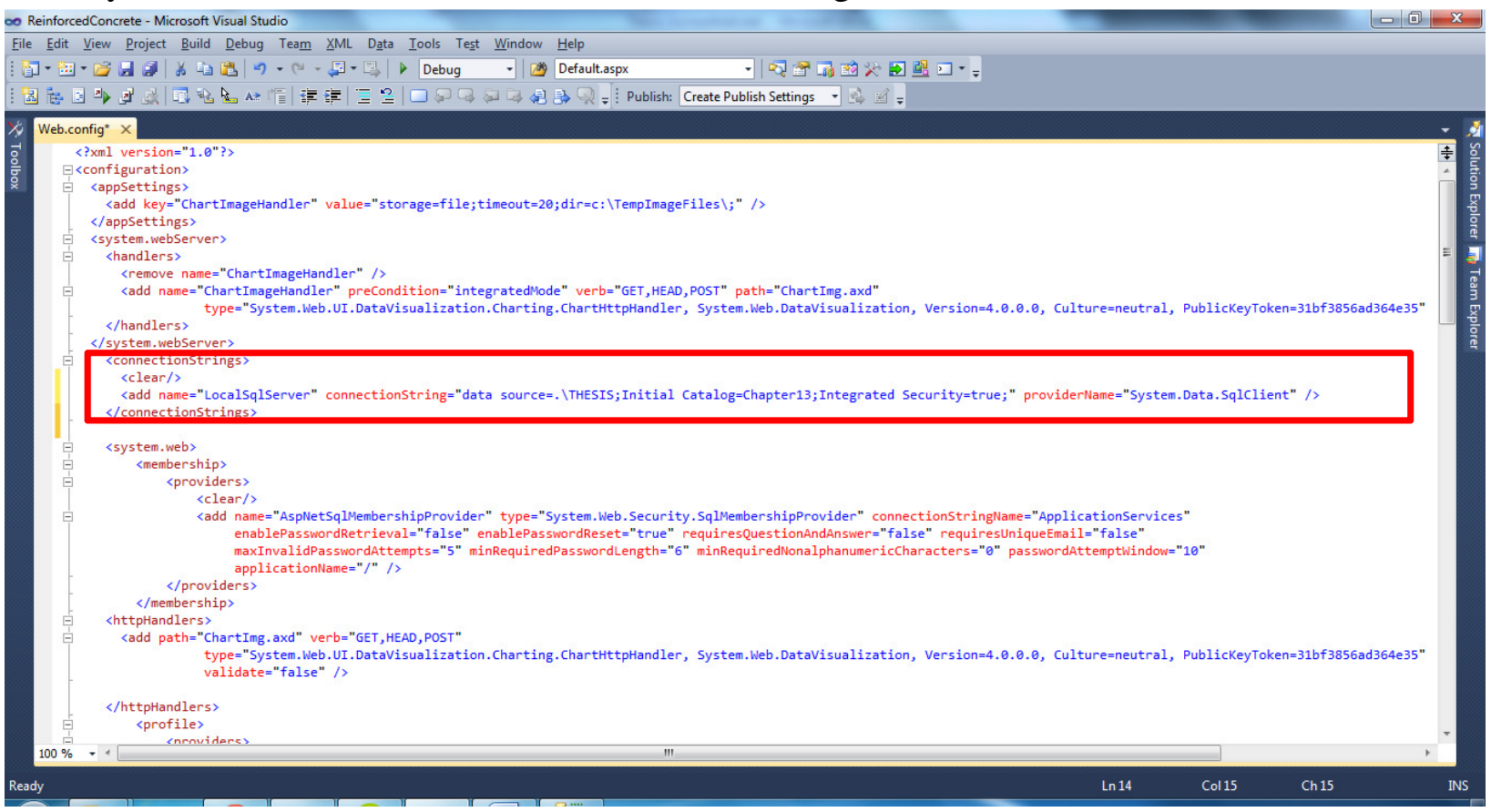

- After configuring database, click F5 to run the application or from menu Debug $>$ start debugging.

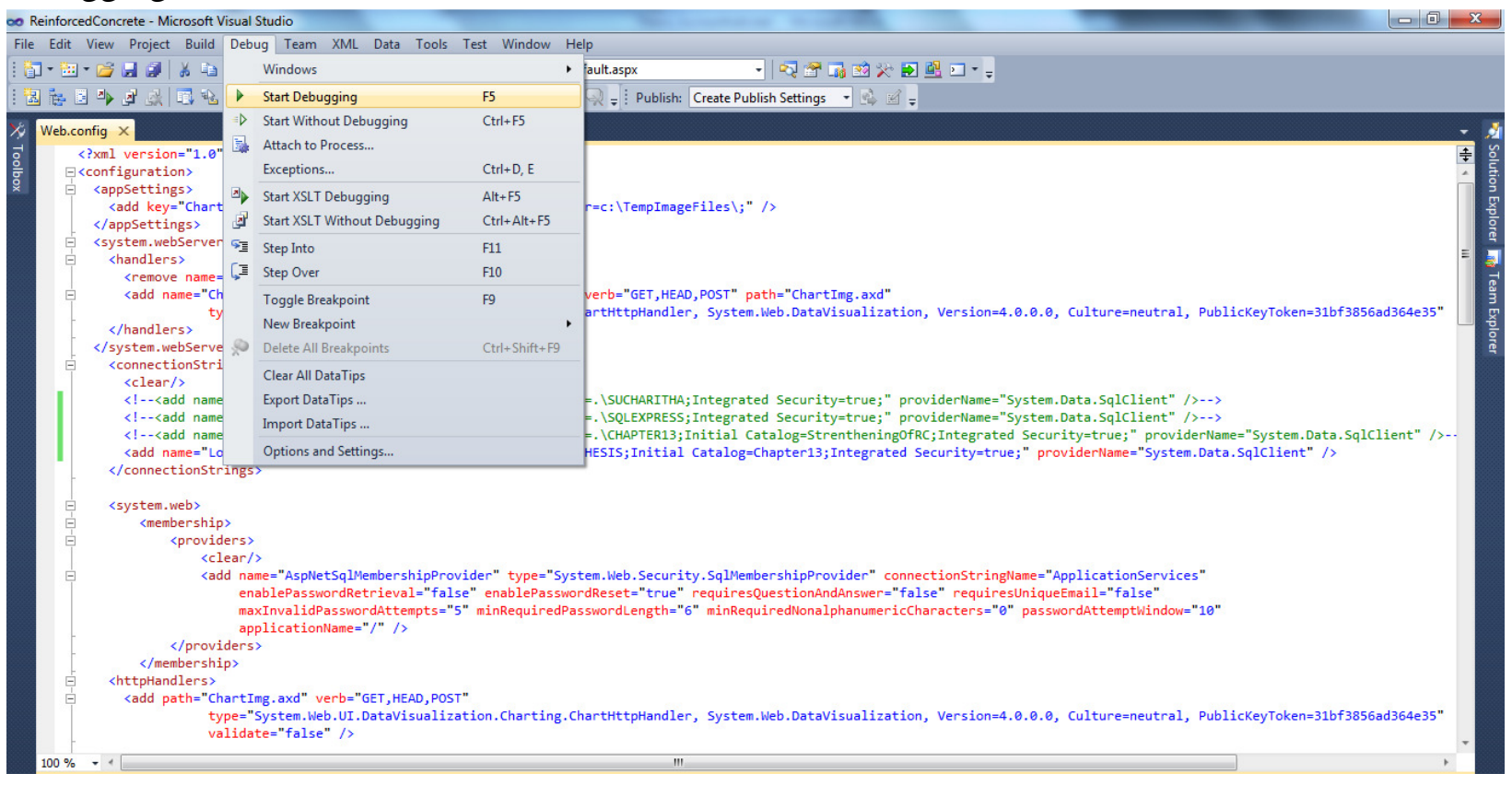


- From home, first create new documents for Load configuration, RC Member Configuration and FRP properties.

$\sqrt{\square \text { Home Page }} \times \square$ Strengthening of Reinfor $\times \square$ localhost:59687

\section{Strengthening of Reinforced Concrete}

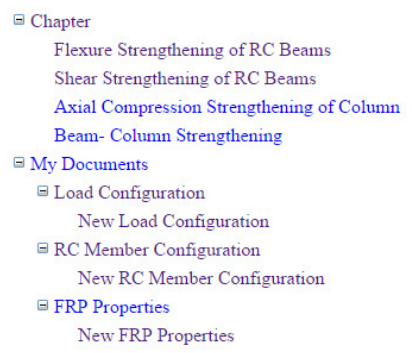

\section{Strengthening of Reinforced Concrete}

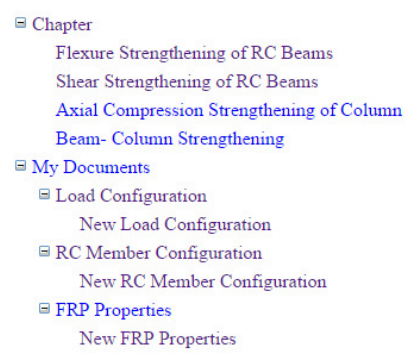

\begin{tabular}{|c|c|c|c|}
\hline Name. & & & \\
\hline RCMemberType: & & \multicolumn{2}{|l|}{ BeamFlexure $\boldsymbol{V}$} \\
\hline$D L:$ & & \multicolumn{2}{|r|}{$66900000 \mathrm{Nmm}$} \\
\hline$L L:$ & & \multicolumn{2}{|r|}{$83000000 \mathrm{Nmm}$} \\
\hline$F_{d}:$ & & \multicolumn{2}{|r|}{$0 \mathrm{Nmm}$} \\
\hline$S_{w}:$ & & \multicolumn{2}{|r|}{$\mathrm{O} \mathrm{Nmm}$} \\
\hline$R_{n}:$ & & \multicolumn{2}{|r|}{$0 \mathrm{Nmm}$} \\
\hline$W_{d}:$ & & \multicolumn{2}{|r|}{$\mathrm{O} N \mathrm{~mm}$} \\
\hline$E_{q}:$ & & \multicolumn{2}{|r|}{$0 \mathrm{Nmm}$} \\
\hline$T_{p}:$ & & \multicolumn{2}{|r|}{$0 \mathrm{Nmm}$} \\
\hline$H_{h}:$ & & \multicolumn{2}{|r|}{$\mathrm{O} \mathrm{Nmm}$} \\
\hline$L_{r}:$ & & \multicolumn{2}{|r|}{$0 \mathrm{Nmm}$} \\
\hline \multirow{3}{*}{ Exposure Conditions } & - Interior & & \\
\hline & Exterior & & \\
\hline & Aggressive & & \\
\hline \multicolumn{4}{|c|}{ H } \\
\hline
\end{tabular}


- List of all load configuration documents created could be seen in My Documents>Load configuration page. Similarly, member configuration and FRP properties can be created and viewed.

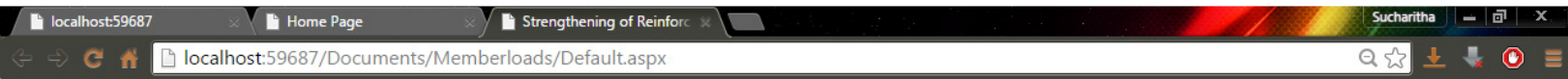

\begin{tabular}{|c|c|c|c|c|c|c|c|c|c|c|c|c|c|}
\hline & \multicolumn{13}{|c|}{ Strengthening of Reinforced Concrete } \\
\hline & Name & $N$ & $N$ & $N$ & & & & & & & $N$ & $N$ & $N$ \\
\hline $\begin{array}{l}\text { B Chapter } \\
\text { Flexure Strengthening of RC Beams }\end{array}$ & (1) ExampleG & 92000 & 130000 & 0 & 0 & & 00 & 0 & 0 & 0 & 0 & 0 & 0 \\
\hline Shear Strengthening of RC Beams & (i) ExampleK & & 0 & 0 & 0 & & 00 & 0 & 0 & 0 & 5200000 & 450000000 & 01 \\
\hline $\begin{array}{l}\text { Axial Compression Strengthening of Column } \\
\text { Beam-Column Strengsthening }\end{array}$ & (ii) ExampleB & 378000000 & 495000000 & 0 & 0 & & 00 & 0 & 0 & 0 & 0 & 0 & 1 \\
\hline $\begin{array}{l}\text { Beam- Column Strengthening } \\
\text { 日 My Documents }\end{array}$ & (ii) ExampleL & & 0 & 0 & 0 & & 00 & 0 & 0 & 0 & 520000 & 45000000 & 0 \\
\hline$\theta$ Load Configuration & (11) 歇 ExampleH & 950000 & 1280000 & 0 & 0 & & 00 & 0 & 0 & 0 & 0 & 0 & 1 \\
\hline $\begin{array}{l}\text { New Load Configuration } \\
\text { Q RC Member Configuration }\end{array}$ & (1) ExampleF & 24400 & 381000 & 0 & 0 & & 00 & 0 & 0 & 0 & 0 & 0 & 0 \\
\hline New RC Member Configuration & (i) ExampleD & 90100000 & 205000000 & 0 & 0 & & 00 & 0 & 0 & 0 & 0 & 0 & 0 \\
\hline \multirow{6}{*}{$\begin{array}{l}\text { G FRP Properties } \\
\text { New FRP Properties }\end{array}$} & (i) ExampleE & 72500 & 56700 & 0 & 0 & & 00 & 0 & 0 & 0 & 0 & 0 & 0 \\
\hline & (ii) ExampleJ & 567000 & 2780000 & 0 & 0 & & 00 & 0 & 0 & 0 & 0 & 0 & 1 \\
\hline & (1) 部 ExampleA & 66980000 & 83350000 & 0 & 0 & & 00 & 0 & 0 & 0 & 0 & 0 & 0 \\
\hline & 9 彭 Test & 66900000 & 83000000 & 0 & 0 & & 00 & 0 & 0 & 0 & 0 & 0 & 0 \\
\hline & (1) Examplec & 100000000 & 176000000 & 0 & 0 & & 00 & 0 & 0 & 0 & 0 & 0 & 0 \\
\hline & (11) 部 ExampleI & 1420000 & 2560000 & 0 & 0 & & 00 & 0 & 0 & 0 & 0 & 0 & 1 \\
\hline
\end{tabular}

- Process to verify procedures can referred from Chapter 6 